\title{
Sterols and Triterpene Diols in Virgin Olive Oil: A Comprehensive Review on Their Properties and Significance, with a Special Emphasis on the Influence of Variety and Ripening Degree
}

\author{
Marina Lukić $^{1, *}$, Igor Lukić $^{1}$ (D) and Tihomir Moslavac ${ }^{2}$ \\ 1 Institute of Agriculture and Tourism, Karla Huguesa 8, 52440 Poreč, Croatia; igor@iptpo.hr \\ 2 Faculty of Food Technology Osijek, Josip Juraj University of Osijek, F. Kuhača 18, 31000 Osijek, Croatia; \\ tihomir.moslavac@ptfos.hr \\ * Correspondence: marina@iptpo.hr; Tel.: +385-52-408-342
}

Citation: Lukić, M.; Lukić, I.; Moslavac, T. Sterols and Triterpene Diols in Virgin Olive Oil: A Comprehensive Review on Their Properties and Significance, with a Special Emphasis on the Influence of Variety and Ripening Degree. Horticulturae 2021, 7, 493. https:/ / doi.org/10.3390/horticulturae7110493

Academic Editors:

Jelena Popović-Djordjević and Luiz Fernando Cappa de Oliveira

Received: 8 October 2021

Accepted: 9 November 2021

Published: 13 November 2021

Publisher's Note: MDPI stays neutral with regard to jurisdictional claims in published maps and institutional affiliations.

Copyright: (c) 2021 by the authors. Licensee MDPI, Basel, Switzerland. This article is an open access article distributed under the terms and conditions of the Creative Commons Attribution (CC BY) license (https:// creativecommons.org/licenses/by/ $4.0 /)$.

\begin{abstract}
Olive oil is considered one of the most valuable vegetable oils and is highly appreciated by consumers for its specific and distinguishable taste and aroma, as well as its nutritional value. Sterols and triterpene diols are important carriers of bioactive properties of olive oil and are responsible for some of the beneficial effects of its consumption on human health, such as lowering serum LDLcholesterol levels and significantly reducing the risk of cardiovascular diseases. The concentration of total sterols and the proportions of particular sterols and triterpene diols are among the parameters used to verify and prove the authenticity of olive oil in accordance with the EU and other countries' regulations. Finally, their composition has been shown to have high discrimination potential for ensuring traceability with respect to variety, geographical origin, harvest date, and other factors. For these reasons, the research on sterols and triterpene diols in olive oil is an ever-growing field of scientific interest with great practical importance. This review focuses on all the important aspects of sterols and triterpene diols in olive oil, from their chemical structure, biosynthesis, occurrence and role in plants, health benefits, and their use in official controls of olive oil purity and authenticity, to a conclusive survey on the recent findings about the effects of different factors of influence on their content and composition, with a detailed comparative analysis of studies that investigated the effects of the two most important factors, variety and ripening degree.
\end{abstract}

Keywords: virgin olive oil; sterols; triterpene diols; EU regulation; trade standards; varietal characterization and differentiation; maturation

\section{Introduction}

Olive oil is obtained from olive fruit (Olea europaea L.) and, when processing of olives involves only mechanical procedures, virgin olive oil is obtained. It is considered one of the most valuable vegetable oils and is highly appreciated by consumers for its specific and distinguishable taste and aroma, as well as for its nutritional value and biological activity; the consumption of olive oil is associated with numerous beneficial effects on the human body and health [1]. Because of all the above, olive oil is today of great economic importance for producer countries, and is thus one of the agri-food products most often subject to forgery and deliberate mislabeling.

Olive oil is an indispensable part of the so-called Mediterranean diet and the culture, history, and tradition of Mediterranean countries. As a plant, olive is typically distributed in the Mediterranean area (more than 95\%; [2]), so the largest growers and producers of olive oil are countries such as Spain, Italy, Greece, Portugal, Turkey, Tunisia, Algeria, and Morocco, and in the last 20 years countries from other continents, such as Australia and North and South America [2-5]. Mediterranean countries account for approximately $90 \%$ and the European Union (EU) for about 70\% of world production worth 7 billion 
euros per year, which makes olive growing and olive oil production an important factor in the agri-food sector and the overall economic and social development of the EU producer countries [4-6].

Olive oil consists mainly of triglycerides, representing more than $98 \%$ of the total oil weight. The remaining, very important part (approximately $2 \%$ ) is the so-called unsaponifiable fraction, composed of over 200 chemical compounds from different groups, which include sterols (phytosterols or 4-desmethyl-sterols) and triterpene diols [7]. Knowledge on the content and composition of the mentioned groups of compounds in olive oil is of great importance.

Besides phenols, tocopherols, and unsaturated fatty acids, sterols and triterpene diols are important carriers of bioactive properties of olive oil and are partly responsible for the beneficial effects of its consumption on human health. Among other biological properties, olive oil sterols have been shown to lower serum LDL-cholesterol levels and significantly reduce the risk of cardiovascular diseases [8,9]. Knowledge of the content and composition of these compounds in virgin olive oil is important for the identification of varieties and geographical areas, as well as specific cultivation and processing practices suitable for obtaining and protecting oils with a higher proportion of these bioactive ingredients as an added value.

The concentration of total sterols and the proportion of particular sterols and triterpene diols are among the parameters used to verify and prove the authenticity of olive oil in accordance with EU regulations [10]. Constant and comprehensive characterization of the content and composition of these groups of compounds in extra virgin olive oils of different varieties and from different geographical locations is important for predicting and avoiding problems in trade due to the possibility of deviation of their chemical composition from the EU regulation, despite their authenticity. This was the case, for example, with the content of campesterol being higher than the maximum permitted percentage of $4 \%$, as well as with the concentration of total sterols being lower than the limit of $1000 \mathrm{mg} / \mathrm{kg}$ in authentic oils from particular varieties, which will be discussed in the following sections. In the EU, many high-quality extra virgin olive oils are additionally valorized by protected designation of origin (PDO) [11]. Each extra virgin olive oil holder of a PDO is produced in accordance with a set of specific requirements prescribed by a holder of a designation in a specification document, which defines various aspects of production and marketing, such as olive varieties used; growing, harvesting, and processing conditions; as well as physico-chemical parameters and sensory properties of produced oils. The conformity of a PDO olive oil with the parameters prescribed in a specification is checked by an accredited certification body. In order to develop robust specifications with the requirements achievable by producer holders of a particular PDO from year to year, which at the same time ensure differentiation from other olive oils, it is necessary to constantly monitor and control all the factors that may affect the sensory quality and chemical composition, including sterols and triterpene diols.

Previous research has shown that the content and composition of sterols and triterpene diols in olive oil are influenced by a number of environmental, agronomic, and technological factors. Although olive processing conditions can have a significant impact [12], they are more controllable and subject to standardization. On the other hand, the influence of variety, often in conjunction with a specific geographical position of olive orchards with associated agro-climatic conditions, has been identified as a key factor with less predictable outcomes regarding the chemical composition. The choice of harvest date, i.e., ripening degree of olive fruits, also has a decisive influence on the sterol composition of olive oil [12-14]. It has been shown that the variability in the contents of certain important constituents of olive oil due to the influence of variety and ripening degree can reach similar proportions. This indicates the possibility that varietal characterization can be compromised under the influence of other factors, and that it is crucial to take them into account when characterizing a particular monovarietal olive oil and its potential.

In this review article, the main representatives of sterols and triterpene diols in olive oil are presented, with information on their chemical structure, biosynthesis, occurrence 
and role in plants, biological activity, and importance in the control of olive oil authenticity and traceability. An overview of the current knowledge about the main factors that affect their content and composition in olive oil is presented, with a special emphasis on the influence of variety and ripening degree, as well as other factors, such as growing season, geographical region, and various agronomic and technological parameters.

\section{Occurrence and Role of Sterols in Plants}

Plant sterols or phytosterols are a group of natural chemical compounds that are formed by biosynthesis in plants. Compared with animal and fungal sterols, they are characterized by several specificities. In animals, the only product of sterol biosynthesis is cholesterol; in fungi, it is ergosterol; while in plants, the biosynthetic pathway results in several different end products that occur in ratios that are genetically predetermined [15]. The term sterols is commonly used for 4-desmethylsterols, which are the most numerous and most represented group of sterols in the plant world, with major representatives $\beta$-sitosterol, $\Delta^{5}$-avenasterol, campesterol, and stigmasterol, followed by other minor sterols [8]. The related groups of 4 -methylsterols and 4,4'-dimethylsterols (also known as triterpene alcohols) are the precursors of sterols (4-desmethylsterols), and are somewhat less represented, although they may occur in concentrations comparable to those of 4-desmethylsterols [16].

Plant sterols occur in both free and bound form. The free forms are important for plant cells because they are an integral part of the structure of the cell membrane and contribute to ensuring its cohesion and fluidity, which helps the plant to maintain its structure under temperature changes [8,17]. They are involved in embryonic growth and lipid accumulation and are important for signal transduction, cell differentiation, and reorganization of cell structure, as well as in defense against infectious diseases $[15,17,18]$. Plant sterols serve as precursors of brassinosteroids, the most important type of plant hormones that regulate plant growth and development $[15,19]$ and have an active role in plant adaptation to biotic and abiotic stress situations [20].

It is considered that steryl esters are formed when free forms are present in excess to keep free sterol levels at physiologically optimal levels. Esterification involves the reaction of the only hydroxyl group in a sterol molecule with a carboxylic group of a molecule of fatty or phenolic acid [15]. Another form of bound sterols are steryl glucosides, which are also thought to be involved in fine-tuning of the concentration of free sterols in the membrane. $\beta$-Sitosteryl glucoside is thought to be involved in the synthesis of cellulose [15]. Steryl glycosides can be further esterified with long-chain fatty acids into acylated steryl glycosides [21]. Sterols are mostly located in cell membranes, while steryl esters mainly fill the intercellular spaces [8].

\section{Molecular Structure of Sterols and Triterpene Diols}

Sterols are considered lipids by nature because of their relatively large, mostly nonpolar, and thus hydrophobic molecular structure, with only a single functional polar hydroxyl group. Because of the hydroxyl group, they are alcohols; because of the isoprene-based structure, they belong to the group of triterpenes (terpenes with 30 carbon atoms); and owing to a specific structure with characteristic four rings and an aliphatic chain, they belong to steroids $[8,22,23]$. Plant sterols are formed in a unique biosynthetic pathway in which 4,4'-dimethylsterols and 4-methylsterols serve as precursors to 4-desmethylsterols. Thus, these three groups of compounds are characterized by certain common structural specificities. The basic structure of plant sterols with $\beta$-sitosterol as the main representative resembles that of cholesterol and is characterized by the following (Figure 1): (I) the basic skeleton that consists of four condensed non-aromatic rings, of which three rings with six carbon atoms and one ring with five carbon atoms; (II) a hydroxyl group on the carbon atom at position 3 (C3) in ring A; (III) a double bond at positions 5 and/or 7 in ring $\mathrm{B}$; and (IV) a side alkyl chain at position 17 in ring $\mathrm{D}$, which may contain an 
ethyl/methyl/ethylidene/methylene group attached to C24 in the molecule. Hydrogenated (saturated) sterols are called stanols [23].

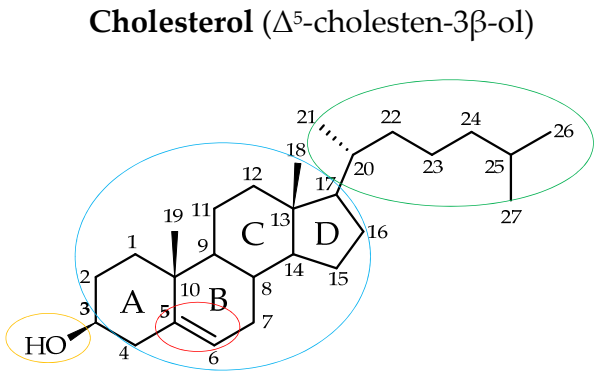

(a)

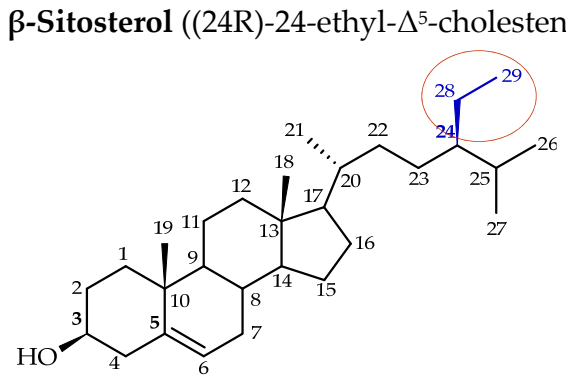

(b)

Figure 1. Chemical structures of (a) cholesterol and (b) $\beta$-sitosterol. Blue ellipse: basic skeleton; orange ellipse: hydroxyl group at C3 carbon atom; red ellipse: double bond at C5 and/or C7 carbon atom; green ellipse: side alkyl chain at C17 carbon atom; brown ellipse: ethyl group at C24 carbon atom.

Sterol compounds are characterized by a stereochemical arrangement of methyl and other substitution groups and hydrogen atoms (Figure 1). Rings A, B, and C are in chair conformation, while ring $\mathrm{D}$ is usually planar. By convention, the hydrogen atoms and other substituent groups are stereochemically arranged relative to the angular methyl group bound to the carbon atom C10. If a plane on which the four rings of the sterol skeleton are laid is imagined, the substituent on C10 is by definition above the plane, so the substituents below the planar surface are said to be $\alpha$-oriented or in trans-conformation, and the substituents above the surface are $\beta$-oriented or in cis-conformation with respect to the angular methyl group at position 10. Thus, in the cholesterol molecule, the hydroxyl group on $\mathrm{C} 3$, the angular methyl group on $\mathrm{C} 13$, the side chain on $\mathrm{C} 17$, and the hydrogen atom on C 8 are $\beta$-oriented (cis), while the hydrogen atoms on carbons 9,14 , and 17 are $\alpha$-oriented (trans). Sterols that are not methylated at position 4 are referred to as desmethylsterols [24].

\section{Sterol and Triterpene Diol Biosynthesis in Plants}

Plant sterols are products of the isoprenoid biosynthetic pathway, which involves a number of enzyme-controlled steps (Figure 2). The basic building blocks of all terpenoids, including sterols, which are triterpenoids (C30), are molecules of isopentenyl pyrophosphate (IPP) (C5) formed in the mevalonic acid (C6) cycle [8,25]. In the next steps, IPP converges to dimethylalkyl diphosphate, which serves as a precursor for the synthesis of monoterpenes, diterpenes, and tetraterpenes, and which is further converted to farnesyl pyrophosphate, from which triterpenes and sesquiterpenes are formed. The enzyme squalene synthase directs the pathway further to sterol synthesis via squalene, while the competitive synthesis of sesquiterpenes from the same substrate is catalyzed by sesquiterpene cyclase. It is considered that a key limiting step for the biosynthesis of plant sterols is the formation of IPP, in which the enzyme 3-hydroxy-3-methyl-glutaryl coenzyme A reductase (HMGR) plays a major role by catalyzing the conversion of acetyl coenzyme A molecules into mevalonic acid [15]. Some authors, on the other hand, question the key role of this enzyme [8]. In the next steps, squalene is converted into 2,3-oxidosqualene, which is cyclized into a triterpene alcohol, i.e., 4,4'-dimethylsterol cycloartenol, a precursor to all plant sterols. Cycloartenol is further transformed into other 4,4'-dimethylsterols, which are converted into 4-methylsterols, and those are finally demethylated to yield end-product 4-desmethylsterols, i.e., sterols, in the following order: $\Delta^{7}$-sterols, $\Delta^{5,7}$-sterols, and $\Delta^{5}$-sterols [15] (Figure 2). At a certain point of the sterol biosynthetic pathway, a bifurcation occurs, catalyzed by two different enzymes, SAM-24-methylene-lophenol-C-24methyltransferase2 (SMT2) and C- $4 \alpha$-sterol-methyl-oxidase2 (SMO2), that, through several additional conversions, leads to the separate formation of major olive oil 24-ethylsterols 
(in biosynthetic order: $\Delta^{5}$-avenasterol, $\beta$-sitosterol, and stigmasterol) and 24-methylsterols (campesterol) from a common precursor gramisterol (Figure 2).

In another branch of the sterol biosynthetic pathway, in several steps, a fraction of 2,3oxidosqualene is transformed into a number of different pentacyclic triterpenes, including $4,4^{\prime}$-dimethylsterols such as $\alpha$ - and $\beta$-amyrin [26], which are further converted to other pentacyclic triterpenoids, including the major olive and olive oil triterpene diols uvaol and erythrodiol, respectively [27] (Figure 2). 


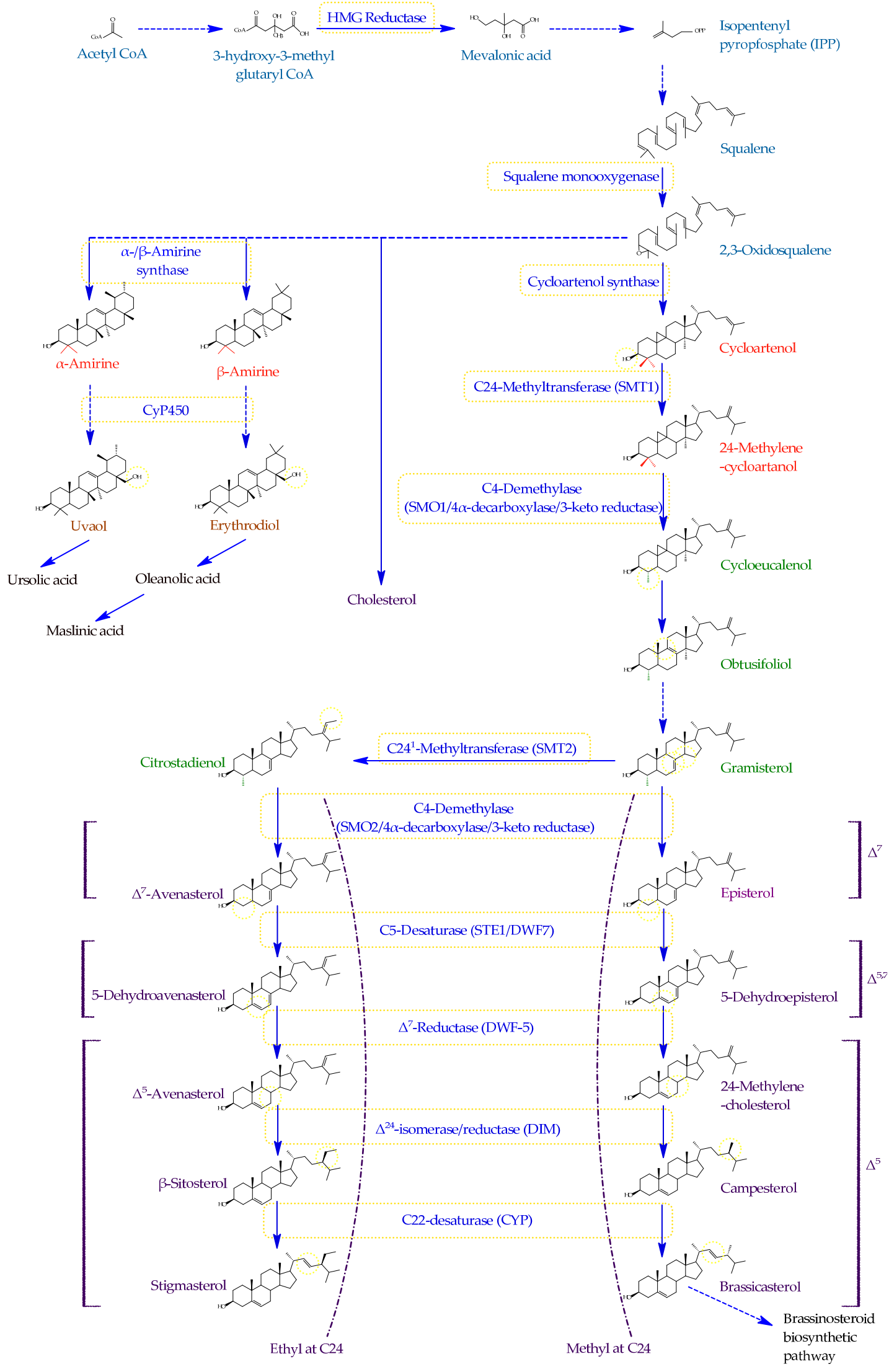

Figure 2. Schematic diagram of the biosynthetic pathway of plant sterols in higher plants $[8,15,27]$ with a special emphasis on sterols found in olive oil, showing the main structures of 4-desmethylsterols (violet), as well as 4-methyl (green) and 4, $4^{\prime}$-dimethylsterols (red) as their biogenetic precursors. Parallel pathways of the synthesis of cholesterol and triterpene diols erythrodiol and uvaol are also shown. Dashed arrows represent several enzymatic steps not shown. 


\section{Health Benefits of Sterols and Triterpene Diols}

The biological activity of sterols and their positive impact on human health have been proven by numerous studies, reviewed in an article by Manai-Djebali and Oueslati [9]. Among beneficial effects, protective activity against arteriosclerosis, gastric ulcer, and various inflammatory processes has been reported [28]. It has been found that sterols can have antitumor activity $[29,30]$. Plant sterols, including sterols from olive oil, are mostly associated with a positive effect on the regulation of the concentration of cholesterol bound to low-density lipoproteins (so-called LDL-cholesterol) in the bloodstream, which has been intensively investigated since the 1950s [31].

\subsection{Plant Sterols and Cholesterol}

LDL-cholesterol accumulates on the walls of blood vessels and creates deposits, so its high concentration in blood is considered one of the main causes of many diseases of the vascular system. On the other hand, HDL-cholesterol (high-density lipoprotein) is higher in density and richer in proteins and, through these molecules, the excess cholesterol from peripheral tissues is carried to the liver, where it does not produce negative effects. Because plant sterols are structurally similar to cholesterol, when ingested within a diet, they compete with LDL-cholesterol for absorption in the digestive system. As a result, LDL-cholesterol absorption is partially blocked and blood cholesterol levels are reduced [8]. It has been shown that a daily dose of $2 \mathrm{~g}$ per day can lower LDL-cholesterol levels by up to $10 \%$ [32]. The natural intake of plant sterols is quite low and varies between 167 and $437 \mathrm{mg}$ per day [33], so the authorities of some countries recommend increasing the plant sterol intake up to $2 \mathrm{~g}$ per day through dietary supplements or foods enriched with plant sterols, with the aim of lowering the concentration of LDL-cholesterol and the incidence of cardiovascular diseases [34]. The effects of plant sterols on blood cholesterol concentration and prevention of cardiovascular diseases have been documented in detail in recent review articles $[9,35,36]$.

\subsection{Other Beneficial Effects of Sterols and Triterpene Diols}

In addition to the effects on lowering cholesterol levels, plant sterols have been shown to have anti-tumor, anti-inflammatory, and antioxidant effects, suggesting their importance in the diet of people with normal LDL-cholesterol concentrations as well [28]. For example, after an increased plant sterol intake, a decrease in proinflammatory cytokine levels, including C-reactive protein (CRP) [37,38], and a decrease in atherosclerotic lesions in mice [38] were found. However, the results of a meta-analysis studying the effect of increased intake of plant sterol-enriched foods on CRP levels published in a review by Rocha et al. [39] indicated a relatively small anti-inflammatory effect, as opposed to a significant decrease in LDL-cholesterol concentration confirmed in the same study.

Numerous published reports on the biological inhibitory effect of plant sterols on lung, stomach, ovary, prostate, liver, and breast tumors have been systematized and consolidated in recent review papers $[29,30]$. It has been hypothesized that plant sterols act through several inhibition mechanisms, such as the inhibition of tumor cell formation and growth, angiogenesis, transmission, and metastasis. They also contribute to tumor cell apoptosis by altering cell membrane structure and function, and by lowering blood cholesterol levels. Increased intake of plant sterols can also increase the activity of antioxidant enzymes, and thus alleviate oxidative stress [29].

Data summarized in a recently published review article confirmed that the intake of plant sterols, in addition to lowering cholesterol levels, also affects the levels of fatty acids, triglycerides, and bile acids, thus alleviating the negative effects of various diseases and conditions, including non-alcoholic fatty liver, inflammatory bowel disease, and obesity. On the other hand, the authors highlighted the need for a certain dose of caution because a connection between plant sterols and exacerbation of certain liver diseases during parenteral nutrition was noted. Likewise, the negative cytotoxic and inflammatory effects of sterol oxidation products have been intensively investigated in recent years [17]. 
Several studies have shown the effect of plant sterols, especially $\beta$-sitosterol, in preventing or alleviating diabetes [40,41]. Numerous pharmacological activities of $\beta$-sitosterol have been covered in recent review articles $[42,43]$.

The major triterpene diols from olive oil have also been found to show biological activity. Erythrodiol exhibited antiproliferative and apoptotic effects against adenocarcinoma [44] and promoted the lowering of LDL-cholesterol levels in human blood [45]. Uvaol has been effective in promoting accelerated and improved wound healing [46] and alleviating inflammatory bowel disease in mice [47], while in humans, it has shown antitumor activity $[27,48]$.

\section{Sterols and Triterpene Diols in Olive Oil}

The most representative sterols (4-desmethylsterols) in olive and olive oil are $\beta$ sitosterol $\left(75-90 \%\right.$ of total sterols), $\Delta^{5}$-avenasterol $(5-20 \%)$, campesterol $(1-4 \%)$, and stigmasterol $(0.5-2 \%)$, although deviations in oils of some cultivars were also recorded. Less abundant sterols in olive oil are clerosterol, sitostanol, 24-methylene-cholesterol, $\Delta^{7}$-avenasterol, $\Delta^{7}$-campesterol, $\Delta^{5,24}$-stigmastadienol, $\Delta^{7}$-stigmastenol, and campestanol, and less commonly occurring sterols are $\Delta^{5,23}$-stigmastadienol, cholesterol, and brassicasterol. Erythrodiol and uvaol occur mainly below $4 \%$ of total sterols $[10,49,50]$.

Besides being important because of their biological activity and beneficial effects on human health, being useful in verifying the authenticity of olive oil, and having a high potential for varietal and geographical origin discrimination (which is all discussed in the following sections), particular authors have assigned sterols some additional properties concerning other aspects of olive oil quality. $\beta$-Sitosterol and $\Delta^{5}$-avenasterol are considered the main carriers of antioxidant activity and other beneficial effects among the sterols from olive oil owing to their abundance and structural specificities $[9,51]$. These two sterols were found to strongly positively correlate with the oxidative stability of olive oil [51,52]. A higher campesterol/stigmasterol ratio has been established as a positive measure of olive oil quality [53], which was confirmed by a positive correlation of stigmasterol with acidity and occurrence of defects in olive oils obtained from stored fruit [54]. An increase in the level of $\Delta^{7}$-stigmastenol was observed under various detrimental conditions, such as olive fly infestation, prolonged olive storage before processing, higher olive pressing temperatures, and oil storage in particular containers [55].

\subsection{Sterols and Triterpene Diols as Parameters of Authenticity}

As sterol biosynthesis is conditioned by plant species, vegetable oils differ from each other according to the profile of sterols, so their composition or relative proportions can be and are used as parameters of purity or authenticity of virgin olive oil. The limits prescribed by the Commission Regulation [10] and International Olive Council (IOC) trade standard [49] for individual sterols and triterpene diols, as well as for the minimum content of total sterols in extra virgin and virgin olive oil categories, are shown in Table 1. The indications about the content of some other important sterols in olive oil not covered by the Commission regulation [10] are also presented to allow better comparison with the sterol composition of other vegetable oils. Table 1 also reports the ranges in which particular sterols naturally occur in various other crude vegetable oils [50].

Deviations from the limit values for particular sterols in olive oil prescribed by the Regulation are interpreted as undeclared presence of foreign oils, which is considered consumer fraud and a legal offense. For example, based on the data presented in Table 1, an elevated cholesterol level in olive oil indicates the presence of animal fat [56] or a palm oil fraction [57]. Tomato seed oil is another example of a plant-derived oil with a high cholesterol content [58]. A higher brassicasterol level is specific to rapeseed oil or rapeseed oil with a reduced erucic acid content (Brassicaceae), so an elevated proportion of this sterol in olive oil indicates illegal blending. Higher campesterol proportion is typical for particular seed oils, while increased stigmasterol and $\Delta^{7}$-stigmastenol levels indicate the presence of oils from the plants of the aster family (Asteraceae or Compositae), such as sunflower and 
saffron $[50,59,60]$. In an exemplary case study, the cumulative campesterol and stigmasterol proportion served as a marker for the detection of only $5 \%$ of vegetable oil of other origin in extra virgin olive oil [61]. The Commission Regulation prescribes the lowest allowable proportion of the so-called apparent or total $\beta$-sitosterol, which represents the sum of the relative proportions of $\beta$-sitosterol, $\Delta^{5,23}$-stigmastadienol, sitostanol, $\Delta^{5}$-avenasterol, $\Delta^{5,24}$-stigmastadienol, and clerosterol. Thus, a high proportion of $\beta$-sitosterol and $\Delta^{5}$ avenasterol with low relative proportions of campesterol and stigmasterol compared with the oils of other mentioned plant species is considered the 'sterol fingerprint' of olive oil. The Commission Regulation has set the minimum limit for total sterols at $1000 \mathrm{mg} / \mathrm{L}$, which was considered a naturally achievable concentration for all cold-pressed olive oils, while lower contents are considered a sign of refining [62]. The relative proportion of erythrodiol and uvaol in the sum of total sterols and these two triterpene diols higher than the maximum allowable limit in edible olive oils of $4.5 \%$ is considered to be a sign of the addition of olive pomace oil or even grape seed oil, as solvent extracts these compounds in higher proportions from the pomace, or more precisely olive fruit skins [50,63].

Hazelnut oil has a composition rather similar to that of olive oil, including triglycerides and fatty acids, as well as sterols, i.e., 4-desmethylsterols. When hazelnut production is in surplus, low priced cold-pressed hazelnut oils can be found on the market and used for illegal blending with olive oil. Recently, the composition of other triterpenoids in olive oil, namely 4,4'-dimethylsterols and 4-methylsterols, was successfully used to differentiate olive oil from hazelnut oil [64]. A similar result was achieved using the composition of free and esterified sterols [65]. Mariani et al. [65] have established an index calculated according to the equation (\% campesterol $\left.\times\left(\% \Delta^{7} \text {-stigmastenol }\right)^{2}\right) /\left(\% \Delta^{7}\right.$-avenasterol) in the esterified sterol fraction, and observed that this ratio is always lower than or equal to one for pure olive oils. Cercaci et al. [66] confirmed the validity of this approach, i.e., the use of the so-called 'Mariani ratio', $R_{\mathrm{MAR}}$. 


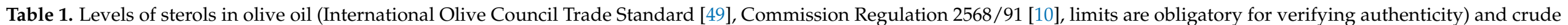

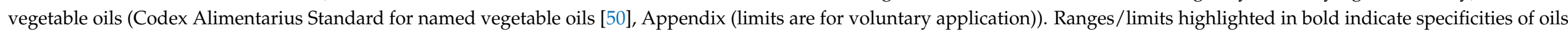
from other plant species with respect to olive oil.

\begin{tabular}{|c|c|c|c|c|c|c|c|c|}
\hline Sterol/Parameter (Unit) & Virgin Olive Oil ${ }^{(1)}$ & $\begin{array}{l}\text { Rapeseed Oil (Low } \\
\text { Erucic Acid) }\end{array}$ & Soyabean Oil & $\begin{array}{l}\text { Sunflower- } \\
\text { Seed Oil }\end{array}$ & $\begin{array}{c}\text { Sunflower- } \\
\text { Seed Oil (High Oleic Acid) }\end{array}$ & Palm Oil & Maize Oil & Hazelnut Oil \\
\hline Cholesterol (\%) & $\leq 0.5$ & $\mathrm{ND}^{(2)}-1.3$ & $0.2-1.4$ & ND-0.7 & ND-0.5 & $2.6-6.7$ & $0.2-0.6$ & ND-1.1 \\
\hline Brassicasterol (\%) & $\leq 0.1$ & $5.0-13.0$ & ND-0.3 & ND-0.2 & ND-0.3 & ND & ND- 0.2 & ND \\
\hline Campesterol (\%) & $\leq 4.0^{(1)}$ & $24.7-38.6$ & $15.8-24.2$ & $6.5-13.0$ & $5.0-13.0$ & $18.7-27.5$ & $16.0-24.1$ & $3.0-6.2$ \\
\hline Stigmasterol (\%) & $<$ Campesterol & $0.2-1.0$ & $14.9-19.1$ & $6.0-13.0$ & $4.5-13.0$ & $8.5-13.9$ & $4.3-8.0$ & ND-2.0 \\
\hline$\beta$-Sitosterol (\%) & (most represented) & $45.1-57.9$ & $47.0-60$ & $50-70$ & $42.0-70$ & $50.2-62.1$ & $54.8-66.6$ & $76.45-96.0$ \\
\hline$\Delta^{5}$-Avenasterol (\%) & (2nd most represented) & $2.5-6.6$ & $1.5-3.7$ & ND-6.9 & $1.5-6.9$ & ND-2.8 & $1.5-8.2$ & $1.0-5.1$ \\
\hline$\Delta^{7}$-Stigmastenol (\%) & $\leq 0.5^{(1)}$ & ND-1.3 & $1.4-5.2$ & $6.5-24.0$ & $6.5-24.0$ & $0.2-2.4$ & $0.2-4.2$ & ND-4.3 \\
\hline$\Delta^{7}$-Avenasterol $(\%)$ & (Not defined) & ND- 0.8 & $1.0-4.6$ & $3.0-7.5$ & ND-9.0 & ND-5.1 & $0.3-2.7$ & ND-1.6 \\
\hline Others (\%) & (Not defined) & $\mathrm{ND}-4.2$ & ND-1.8 & ND-5.3 & $3.5-9.5$ & ND & ND-2.4 & ND \\
\hline Total sterols $(\mathrm{mg} / \mathrm{kg})$ & $\geq 1000$ & $4500-11,300$ & $1800-4500$ & $2400-5000$ & $1700-5200$ & $300-700$ & $7000-22,100$ & $1200-1800$ \\
\hline Erythrodiol + uvaol (\%) & $\leq 4.5$ & (Not defined) & (Not defined) & (Not defined) & (Not defined) & (Not defined) & (Not defined) & (Not defined) \\
\hline
\end{tabular}

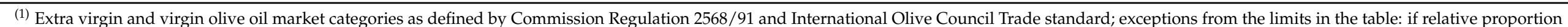

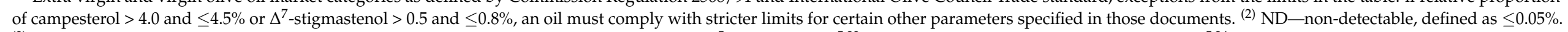

(3) App. $\beta$-Sitosterol-apparent $\beta$-sitosterol is the sum of relative proportions of $\beta$-sitosterol, $\Delta^{5}$-avenasterol, $\Delta^{5,23}$-stigmastadienol, clerosterol, sitostanol, and $\Delta^{5,24}$-stigmastadienol. 


\subsection{Methods of Analysis of Sterols and Triterpene Diols}

The method of analysis of sterols in olive oil used in the majority of studies is a standard method of the IOC applied in the member countries, its current version being the fifth revision [67]. This method is also adopted as the official one by the Commission Regulation of the European Union 2568/91 and later amendments [10], and is used for official controls of olive oil on the EU market. It is also applied in the real sector, i.e., in laboratories within companies that produce or pack and sell olive oil. The IOC method is referenced by the international Codex Alimentarius standard for olive oils and olive pomace oils 'CODEX STAN 33-1981' [68], which is applied in international trade and for regulation of olive oil market in countries that are predominantly importers (not EU). This method has also been adopted by the International Organization for Standardization (ISO) in the standard method 'ISO 12228, Part 2' [69], whereby the ISO method for determination of individual and total sterols contents in olive oils and olive pomace oils became technically identical to the IOC standard method [67]. The American Oil Chemists' Society (AOCS) 'Official Method Ch 6-91' consists of the same procedures as well [70]. Besides being used for testing the authenticity of olive oil on the market, the IOC method [67] adopted as the official one by the Commission Regulation of the EU 2568/91 [10] was by far the most widely used in scientific research on sterols and triterpene diols in olive oil.

The official method $[10,67]$ consists of the following steps:

1. Saponification and extraction of the unsaponifiable fraction of olive oil by liquidliquid extraction with diethyl ether, followed by additional dealkalization and dehydration of the extract;

2. Separation of sterols and triterpene diols from other groups of compounds of the unsaponifiable fraction (aliphatic and triterpenic alcohols, tocopherols, polyphenols) by thin layer chromatography (TLC) using silica gel plates;

3. Elution of sterols and triterpene diols from the corresponding silica band by organic solvent, derivatization into trimethylsilyl ethers, and gas chromatographic (GC) separation;

4. Detection of sterols and triterpene diols using a gas chromatograph with a flame ionization detector and identification by comparison with a reference chromatogram from the official method and retention times relative to an internal standard;

5. Quantification based on an internal standard ( $\alpha$-cholestanol or betulin) added into the sample at the beginning of analysis, assuming a response factor equal to one.

As a result of the analysis, the concentration of total sterols $(\mathrm{mg} / \mathrm{kg})$ and relative proportions (\%) of individual sterols in relation to the sum of all sterols are reported. Relative proportions can be calculated from the concentrations or simply from peak areas, i.e., it is possible to use the internal standard to express the concentrations of each sterol contained in the analyzed olive oil $(\mathrm{mg} / \mathrm{kg})$, although this is not a requirement of the standard method or regulation. The method allows the analysis of 14 main sterols together with erythrodiol and uvaol, while it is also possible to identify and quantify the two sterols that are not characteristic for olive oil, brassicasterol and ergosterol.

Owing to a large number of long preparatory steps before chromatographic analysis, the official method [67] is rather time consuming. It includes the use of relevant quantities of particular toxic chemicals and produces a significant amount of hazardous waste. Furthermore, the method requires a higher level of staff training and laboratory equipment, so it can be concluded that it is rather complex and expensive. For these reasons, efforts have been made to simplify the procedure and reduce the amount of hazardous solvents. On the other hand, the method is very robust if the analytical procedure is applied fully and correctly and has very good validation indicators, such as relative standard deviations in repeatability and reproducibility conditions [67], so it remains the reference method for the analysis of sterols in olive oil up to date.

Several research groups have tried to bypass the step of TLC separation and apply direct silylation of the unsaponifiable fraction, after which the trimethylsilyl ethers were 
analyzed by GC [71-74]. In most cases, such methods reported only major sterols together with other compounds from the unsaponifiable fraction.

Methodologies based on GC $[75,76]$ and GC $\times$ GC $[77,78]$ separation with powerful mass spectrometric (MS) detection have also been proposed, often developed for simultaneous determination of sterols and triterpene diols together with various other compounds from the olive oil unsaponifiable fraction in the so-called multi-class approach. These methods exhibited a number of advantages, such as simpler sample preparation, increased sensitivity, and ability to analyze various compound classes. As far as sterols were concerned, these methods were mostly focused only on the major ones. Chromatographymass spectrometry (C-MS) metabolomics-based methods for the analysis of bioactive compounds from olive oil, including sterols and triterpene diols, were recently summarized in a detailed review article by Olmo-García et al. [79].

Liquid chromatography (LC) has been used for the separation of various groups of olive oil unsaponifiables for more than 20 years [80]. In the last revision of the IOC method [67], separation by preparative LC using a silica gel column followed by ultraviolet or refractive index detection was proposed as an alternative to TLC as the reference separation technique. Several research groups developed methods for separation and analysis of individual sterols by high performance liquid chromatography (HPLC). For example, Cañabate-Díaz et al. [81] followed the same saponification and separation steps as proposed by the official method [10,67], but analyzed sterols without silylation by HPLC-MS. Similar methods were proposed by other authors [82-84] who also used the procedures prescribed in the official method or similar and applied HPLC-MS. These methods allowed the analysis of a fewer number of sterols than the official one, with some of them not completely separated by LC in some cases. Lerma-García et al. [85] applied UPLC-MS for the separation of sterols and detected very low concentrations of ergosterol, which is regularly not identified nor reported, but in this case, served as a good varietal differentiator between monovarietal olive oils.

In recent years, the use of potent instrumentation and the application of the metabolomics approach allowed the identification and relative quantification of a much larger number of sterol compounds in olive oil, which certainly represents one of the most promising tools and directions in the future research on this topic. For example, after a simple extraction in $80 \%$ methanol $(v / v)$ and analysis by ultra-high-pressure liquid chromatography with electrospray quadrupole-time-of-flight hybrid mass spectrometry (UHPLC-ESI/QTOF-MS), particular research groups putatively annotated several hundreds of sterols [86,87].

Alternative hybrid LC-GC-based methods for the analysis of sterols in olive oil proposed to date have been discussed in a recent review article by Conte et al. [63]. Such methods were characterized by a shorter duration and reduced use of toxic solvents, as well as increased sensitivity with low risk of sample contamination, but required highly specialized instrumentation. The quantitative results obtained by the most recent version, which included fully automated saponification and extraction followed by LC-GC analysis without previous derivatization, were fully comparable with the ISO method [88].

Some other techniques also turned out to be successful in detecting and quantifying total and/or particular groups of sterols in olive oil, such as nuclear magnetic resonance (NMR) [89], Fourier-transform near infrared (FT-NIR) spectroscopy [90], and others.

Sterols in olive and olive oil occur in free and bound form, esterified to fatty or phenolic acids, or bound to sugar molecules into glycosides that may or may not be acylated. Information on the proportions of free sterols and steryl esters is lost by conventional sterol analysis, as it involves saponification in the early phase of sample preparation [91]. It has been shown that there is a possibility that information on the proportion and amounts of sterols in free and bound form may be useful for several purposes. The ratio of sterols in free and bound form can be used to distinguish vegetable oils of different origin [92,93]. It has also been shown that the analysis of free and esterified sterol forms can indicate the presence of hazelnut oil in olive oil [94], which is a potential solution to a major problem, given that hazelnut and olive oil can hardly be distinguished by the composition of triacylglycerols, 
fatty acids, or sterols using the current official method, defined parameters, and the limits prescribed by the Regulation [10].

In studies in which the proportions of free and bound sterols were determined, solidphase extraction (SPE), using mostly silica gel columns, was used for sample preparation before chromatographic analysis. In a step preceding saponification and separation by TLC, Mariani et al. [94] have isolated esterified sterols in the nonpolar fraction using a silica gel column and elution with a mixture of n-hexane and diethyl ether, while free sterols were eluted subsequently in the polar fraction with diethyl ether. Cunha et al. [95] also separated esterified from free sterols by SPE. Esterified sterols were eluted first with n-hexane/ethyl acetate $(90: 10, v / v)$, while n-hexane/ethyl acetate $(90: 10, v / v)$ followed by ethanol/diethyl ether/n-hexane (50:25:25, $v / v / v)$ solvent mixture was used for the elution of free forms. In all the mentioned cases, separate fractions of free and bound sterols were silylated and subjected to GC analysis. Valli et al. [96] validated a simplified method protocol for simultaneous analysis of free and esterified sterols, triterpene alcohols, and $\alpha$-tocopherols by direct silylation in oil followed by SPE and GC analysis. The proposed method was successful in detecting simulated adulteration of pure extra virgin olive oil by sunflower oil based on the ratio between the free and esterified forms.

Gómez-Coca et al. [97] developed a method for simultaneous determination of free and esterified steryl glucosides. After SPE and elution with chloroform/methanol, glucosides were analyzed both by (i) hydrolysis of esterified steryl glucosides followed by separate GC-FID analysis of the released fatty acids and derivatized steryl glycosides, and by (ii) direct analysis of individual esterified steryl glucosides by normal phase and reverse phase HPLC-MS.

\section{Factors Affecting the Content and Composition of Sterols and Triterpene Diols in Olive Oil}

The content and composition of sterols and triterpene diols in olive oil are influenced by a number of environmental, agronomic, and technological factors. Knowledge on how particular conditions and procedures affect sterols and triterpene diols may allow better control and management of the whole process from field to table to come as close as possible to obtaining tailored products with desired composition and quality. In this review, all the main factors were considered, with a special emphasis on the influence of olive variety and ripening degree.

\subsection{Influence of Variety}

The selected studies that investigated the effect of olive variety on sterol and triterpene diol contents and composition in olive oil up to date are listed in Table 2. Information includes the country of origin of the studied oils, varieties, statistical methods used for varietal differentiation, the most significant varietal differentiators among sterols or triterpene diols if specified, and deviations from the limits set by legislation [10] and the trade standard [49]. For each study, additional details were provided, such as the number of harvest years, growing regions, ripening degree and ripening index (RI), olive processing method and sample type, total number of samples, an indication if a multivariate chemometric varietal differentiation was achieved, concentration ranges of total sterols and proportions of total triterpene diols, and other relevant information. 
Table 2. Virgin olive oil varietal characterization and differentiation studies based on the content and composition of sterols and triterpene diols.

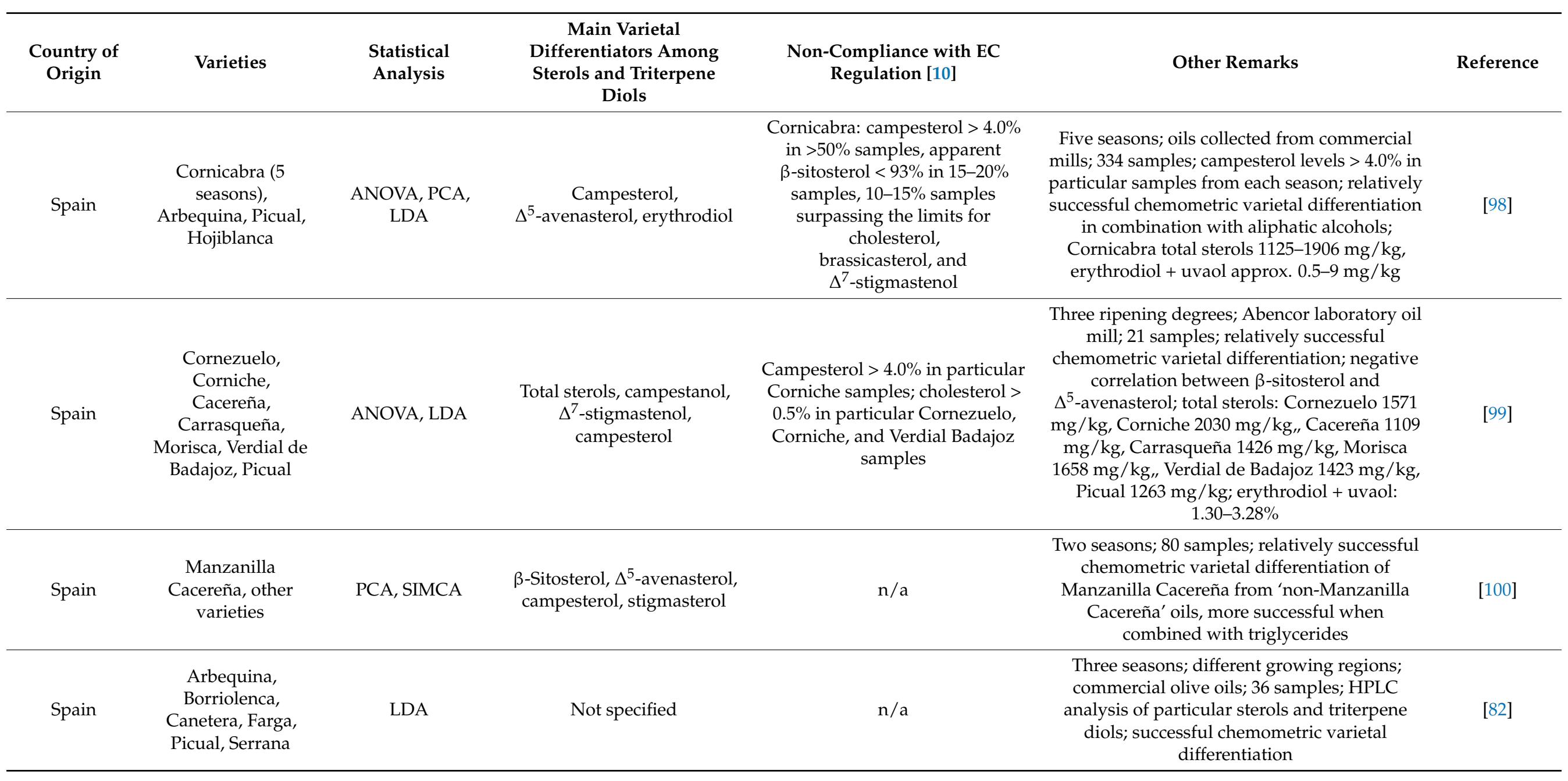


Table 2. Cont.

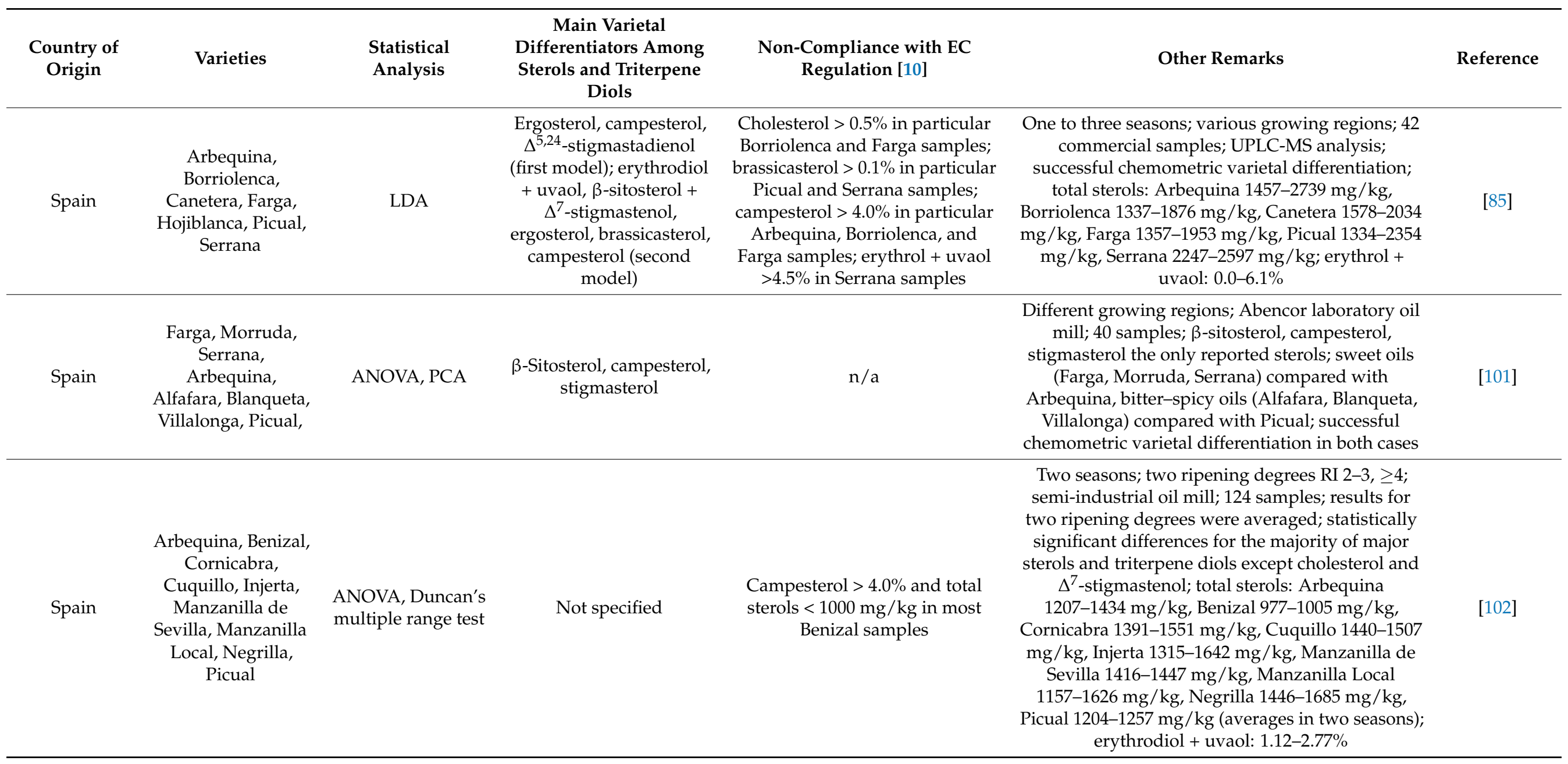


Table 2. Cont.

\begin{tabular}{|c|c|c|c|c|c|c|}
\hline $\begin{array}{c}\text { Country of } \\
\text { Origin }\end{array}$ & Varieties & $\begin{array}{c}\text { Statistical } \\
\text { Analysis }\end{array}$ & $\begin{array}{l}\text { Main Varietal } \\
\text { Differentiators Among } \\
\text { Sterols and Triterpene } \\
\text { Diols }\end{array}$ & $\begin{array}{c}\text { Non-Compliance with EC } \\
\text { Regulation [10] }\end{array}$ & Other Remarks & Reference \\
\hline Spain & $\begin{array}{c}\text { Cornicabra, } \\
\text { Manzanilla } \\
\text { Cacereña, } \\
\text { Manzanilla } \\
\text { Castellana, Picual }\end{array}$ & $\begin{array}{l}\text { ANOVA, Tukey's } \\
\text { honest test }\end{array}$ & $\begin{array}{l}\text { (Esterified) } \beta \text {-sitosteryl } \\
\text { and } \Delta^{5} \text {-avenasteryl } \\
\text { glucosides }\end{array}$ & $\mathrm{n} / \mathrm{a}$ & $\begin{array}{l}\text { Eight growing regions; two farming modalities } \\
\text { (conventional, organic); four ripening degrees; } \\
\text { Abencor laboratory oil mill; } 44 \text { samples; analysis of } \\
\text { esterified and glucosylated sterols; statistically } \\
\text { significant differences for the majority of analyzed } \\
\text { glucosides (campesteryl, } \beta \text {-sitosteryl, } \\
\Delta^{5} \text {-avenasteryl) and esterified glucosides } \\
\text { ( } \beta \text {-sitosteryl, } \Delta^{5} \text {-avenasteryl); (esterified) } \\
\beta \text {-sitosteryl glucoside the most abundant; } \\
\text { (esterified) stigmasteryl glucoside mostly not } \\
\text { detected; total steryl glucosides: } 0.59-2.62 \mathrm{mg} / \mathrm{kg} \text {; } \\
\text { total esterified steryl glucosides: } 0.18-3.07 \mathrm{mg} / \mathrm{kg}\end{array}$ & [103] \\
\hline Spain & $\begin{array}{c}\text { Morisca, } \\
\text { Carrasqueña }\end{array}$ & $\begin{array}{l}\text { ANOVA, Tukey's } \\
\text { multiple range test }\end{array}$ & Not specified & None & $\begin{array}{l}\text { Three seasons; spotted stage of ripening; Abencor } \\
\text { laboratory oil mill; } 54 \text { samples, three ripening } \\
\text { degrees RI 0-2, 2-3, and 3-4 and nine industrial oil } \\
\text { mills, } 162 \text { samples; statistically significant } \\
\text { differences for particular sterols; interaction } \\
\text { between variety and year significant for particular } \\
\text { sterols; negative correlation between } \beta \text {-sitosterol } \\
\text { and } \Delta^{5} \text {-avenasterol; total sterols: Morisca } \\
\text { 1681-1689 mg/kg, Carrasqueña } 1492-1551 \mathrm{mg} / \mathrm{kg} \text {; } \\
\text { erythrodiol + uvaol: } 2.74-3.86 \%\end{array}$ & [104] \\
\hline Spain & $\begin{array}{c}\text { Arbequina, Benizal, } \\
\text { Cornicabra, } \\
\text { Cuquillo, Injerta, } \\
\text { Manzanilla Local, } \\
\text { Manzanilla de } \\
\text { Sevilla, Negrilla, } \\
\text { Picual }\end{array}$ & $\begin{array}{l}\text { ANOVA, Duncan's } \\
\text { multiple range test, } \\
\text { PCA }\end{array}$ & Not specified & $\begin{array}{c}\text { Campesterol }>4.0 \% \text { in most and } \\
\text { total sterols }<1000 \mathrm{mg} / \mathrm{kg} \text { in all } \\
\text { Benizal samples }\end{array}$ & $\begin{array}{l}\text { Four seasons; RI 2-3; semi-industrial oil mill; } 50 \\
\text { samples; relatively successful chemometric varietal } \\
\text { differentiation in combination with FAMEs and } \\
\text { oxidative stability; no quantitative data for sterols }\end{array}$ & [105] \\
\hline
\end{tabular}


Table 2. Cont.

\begin{tabular}{|c|c|c|c|c|c|c|}
\hline $\begin{array}{l}\text { Country of } \\
\text { Origin }\end{array}$ & Varieties & $\begin{array}{l}\text { Statistical } \\
\text { Analysis }\end{array}$ & $\begin{array}{c}\text { Main Varietal } \\
\text { Differentiators Among } \\
\text { Sterols and Triterpene } \\
\text { Diols }\end{array}$ & $\begin{array}{c}\text { Non-Compliance with EC } \\
\text { Regulation [10] }\end{array}$ & Other Remarks & Reference \\
\hline Spain & $\begin{array}{l}\text { Thirty-one minor } \\
\text { cultivars }\end{array}$ & $\begin{array}{l}\text { ANOVA, PCA, } \\
\text { LDA }\end{array}$ & $\begin{array}{c}\text { Apparent } \beta \text {-sitosterol, } \\
\text { campesterol, } \\
\text { stigmasterol }\end{array}$ & $\begin{array}{c}\text { Campesterol }>4.0 \% \text { in } \\
\text { Borriolenca, Vallesa, and } \\
\text { Blanqueta Enguera samples; } \\
\text { apparent } \beta \text {-sitosterol }<93 \% \text { in } \\
\text { Borriolenca and Vallesa samples }\end{array}$ & $\begin{array}{l}\text { Eleven growing regions; RI 2-3; Abencor } \\
\text { laboratory oil mill; } 155 \text { samples; relatively } \\
\text { successful chemometric varietal differentiation in } \\
\text { combination with tocopherols, phenols, FAMEs, } \\
\text { and oil yield }\end{array}$ & [106] \\
\hline Spain & $\begin{array}{l}\text { Forty-three world } \\
\text { varieties }\end{array}$ & PCA, CA & $\begin{array}{c}\beta \text {-Sitosterol, } \\
\Delta^{5} \text {-avenasterol }\end{array}$ & $\begin{array}{c}\text { Cholesterol }>0.5 \% \text { in samples } \\
\text { from two varieties; campesterol }> \\
4.0 \% \text { in samples from five } \\
\text { varieties; apparent } \beta \text {-sitosterol }< \\
93 \% \text { in samples from } 10 \text { varieties; } \\
\Delta^{7} \text {-stigmastenol }>0.5 \% \text { in } \\
\text { samples from a single variety; } \\
28 \% \text { samples outside the limits }\end{array}$ & $\begin{array}{l}\text { Varieties from the World Olive Germplasm Bank, } \\
\text { IFAPA Centro 'Alameda de Obispo', Cordoba, } \\
\text { various countries of origin; same growing and oil } \\
\text { extraction conditions; RI around 3; Abencor } \\
\text { laboratory oil mill; great variability; successful } \\
\text { differentiation of groups of cultivars based on their } \\
\beta \text {-sitosterol and } \Delta^{5} \text {-avenasterol proportions; } \\
\quad \beta \text {-sitosterol } 672 \mathrm{mg} / \mathrm{kg}-2035 \mathrm{mg} / \mathrm{kg} \text {, } \\
\Delta^{5} \text {-avenasterol } 11 \mathrm{mg} / \mathrm{kg}-318 \mathrm{mg} / \mathrm{kg} \text {, total sterols: } \\
\text { Khashabi } 848 \mathrm{mg} / \mathrm{kg} \text {-Sevillenca } 2419 \mathrm{mg} / \mathrm{kg}\end{array}$ & [108] \\
\hline
\end{tabular}


Table 2. Cont.

\begin{tabular}{|c|c|c|c|c|c|c|}
\hline $\begin{array}{l}\text { Country of } \\
\text { Origin }\end{array}$ & Varieties & $\begin{array}{l}\text { Statistical } \\
\text { Analysis }\end{array}$ & $\begin{array}{l}\text { Main Varietal } \\
\text { Differentiators Among } \\
\text { Sterols and Triterpene } \\
\text { Diols }\end{array}$ & $\begin{array}{c}\text { Non-Compliance with EC } \\
\text { Regulation [10] }\end{array}$ & Other Remarks & Reference \\
\hline Spain & $\begin{array}{l}\text { Forty world } \\
\text { varieties }\end{array}$ & PCA, CA & $\begin{array}{c}\text { Erythrodiol ( } 96 \% \text { of } \\
\text { variance), uvaol ( } 4 \% \text { of } \\
\text { variance) }\end{array}$ & $\begin{array}{l}\text { Erythrodiol + uvaol }>4.5 \% \text { in } \\
\text { particular Lechin de Granada } \\
\quad \text { and Moraiolo samples }\end{array}$ & $\begin{array}{l}\text { Varieties from the World Olive Germplasm Bank } \\
\text { Collection of Cordoba, various countries of origin; } \\
\text { same growing and oil extraction conditions; RI } \\
\text { around 3; Abencor laboratory oil mill; great } \\
\text { variability; successful differentiation of groups of } \\
\text { cultivars based on their triterpene diol content; } \\
\text { erythrodiol: Frantoio } 5.89 \mathrm{mg} / \mathrm{kg}-\mathrm{Nevado} \text { Azul } \\
73.78 \mathrm{mg} / \mathrm{kg} \text {, uvaol: Genovesa } 1.50 \mathrm{mg} / \mathrm{kg} \text {-Dolce } \\
\text { Agogia } 19.35 \mathrm{mg} / \mathrm{kg} \text {; erythrodiol + uvaol: St. } \\
\text { George Greys } 0.46 \% \text {-Lechin de Granada } 4.36 \%\end{array}$ & [109] \\
\hline $\begin{array}{l}\text { Spain, Greece, } \\
\text { Italy }\end{array}$ & $\begin{array}{l}\text { Coratina, Picual, } \\
\text { Koroneiki }\end{array}$ & $\begin{array}{l}\text { ANOVA, Duncan's } \\
\text { multiple range test, } \\
\text { correlation, } \\
\text { HCA, CDA }\end{array}$ & $\begin{array}{c}\text { Campesterol, } \\
\beta \text {-sitosterol, } \\
\Delta^{7} \text {-avenasterol }\end{array}$ & $\begin{array}{l}\text { Total sterols < } 1000 \mathrm{mg} / \mathrm{kg} \text { in } \\
\text { particular Koroneiki samples }\end{array}$ & $\begin{array}{l}\text { Two seasons; similar ripening degree; laboratory } \\
\text { oil mill; } 12 \text { samples; statistically significant } \\
\text { differences for all sterols and triterpene diols; } \\
\text { successful chemometric varietal differentiation in } \\
\text { combination with aliphatic alcohols, phenols, } \\
\text { triglycerides, basic quality parameters; negative } \\
\text { correlation between } \beta \text {-sitosterol and } \\
\Delta^{5} \text {-avenasterol; total sterols: Coratina } 1090-1326 \\
\text { mg/kg, Picual } 1646-1882 \mathrm{mg} / \mathrm{kg} \text {, Koroneiki } \\
999-1235 \mathrm{mg} / \mathrm{kg} \text {; erythrodiol + uvaol: } 0.14-3.28 \%\end{array}$ & [110] \\
\hline Italy & $\begin{array}{l}\text { Coratina, } \\
\text { Provenzale, } \\
\text { Frantoio, Moraiolo, } \\
\text { Bosana, Dritta }\end{array}$ & $\begin{array}{l}\text { ANOVA, linear } \\
\text { regression, PCA }\end{array}$ & $\begin{array}{l}\text { Fatty acids, aliphatic, } \\
\text { and triterpenic alcohols } \\
\text { more useful than sterols }\end{array}$ & $\mathrm{n} / \mathrm{a}$ & $\begin{array}{l}\text { Four growing regions; laboratory oil mill; } 63 \\
\text { samples; relatively successful chemometric varietal } \\
\text { differentiation combined with aliphatic and } \\
\text { triterpenic alcohols and fatty acids }\end{array}$ & [71] \\
\hline Italy & $\begin{array}{l}\text { Leccino, Dritta, } \\
\text { Caroleo, Coratina, } \\
\text { Castiglionese, } \\
\text { Carboncella, } \\
\text { Nebbio }\end{array}$ & $\begin{array}{l}\text { ANOVA, PCA, } \\
\text { CDA, HCA, RDA, } \\
\text { KNN }\end{array}$ & Not specified & $\mathrm{n} / \mathrm{a}$ & $\begin{array}{l}\text { Experimental orchard; laboratory oil mill; } 14 \\
\text { samples; successful chemometric varietal } \\
\text { differentiation; average total sterols: } 1847 \mathrm{mg} / \mathrm{kg} \text {; } \\
\text { average erythrodiol + uvaol: } 150 \mathrm{mg} / \mathrm{kg}\end{array}$ & [111] \\
\hline
\end{tabular}


Table 2. Cont.

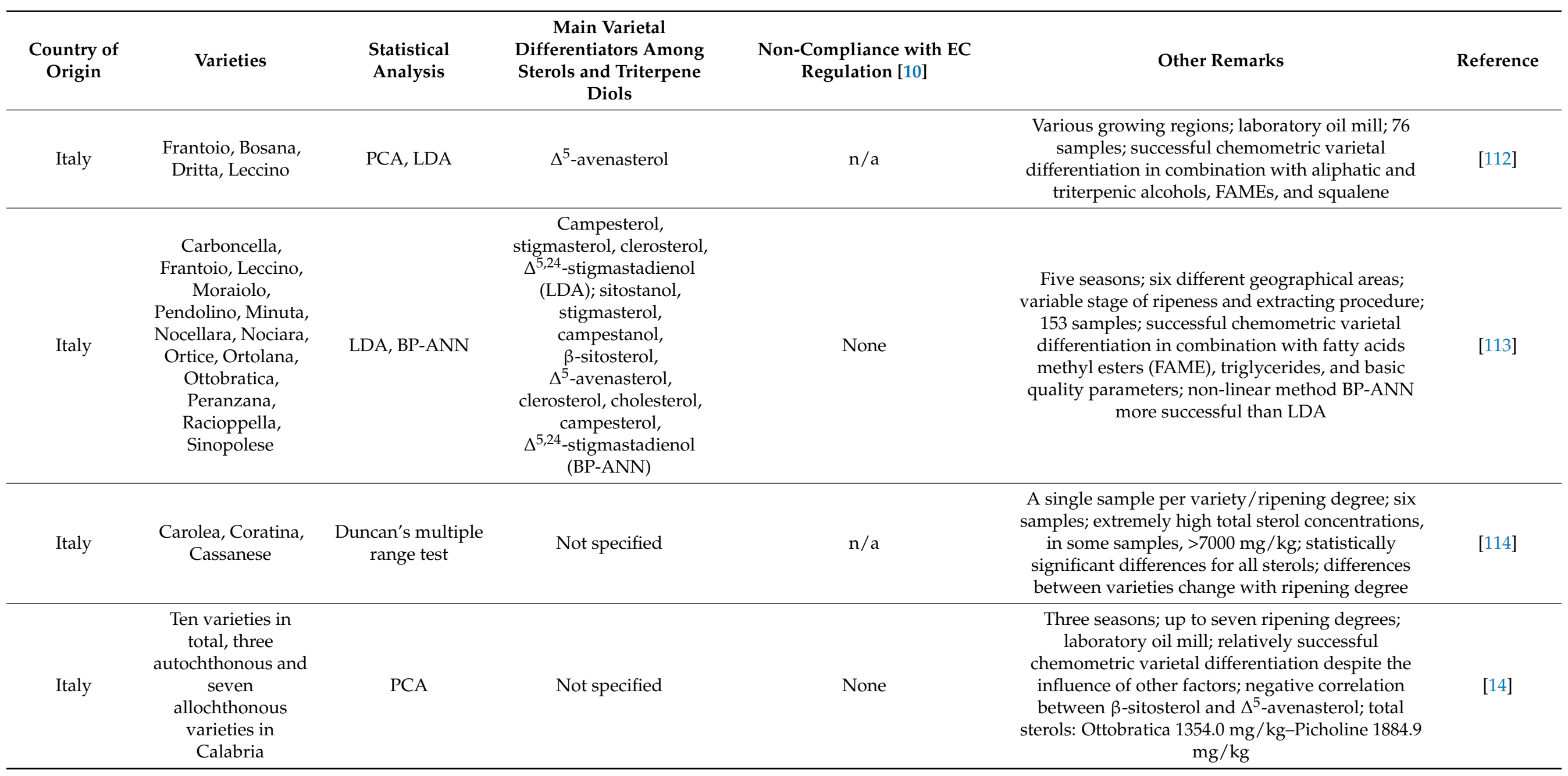


Table 2. Cont.

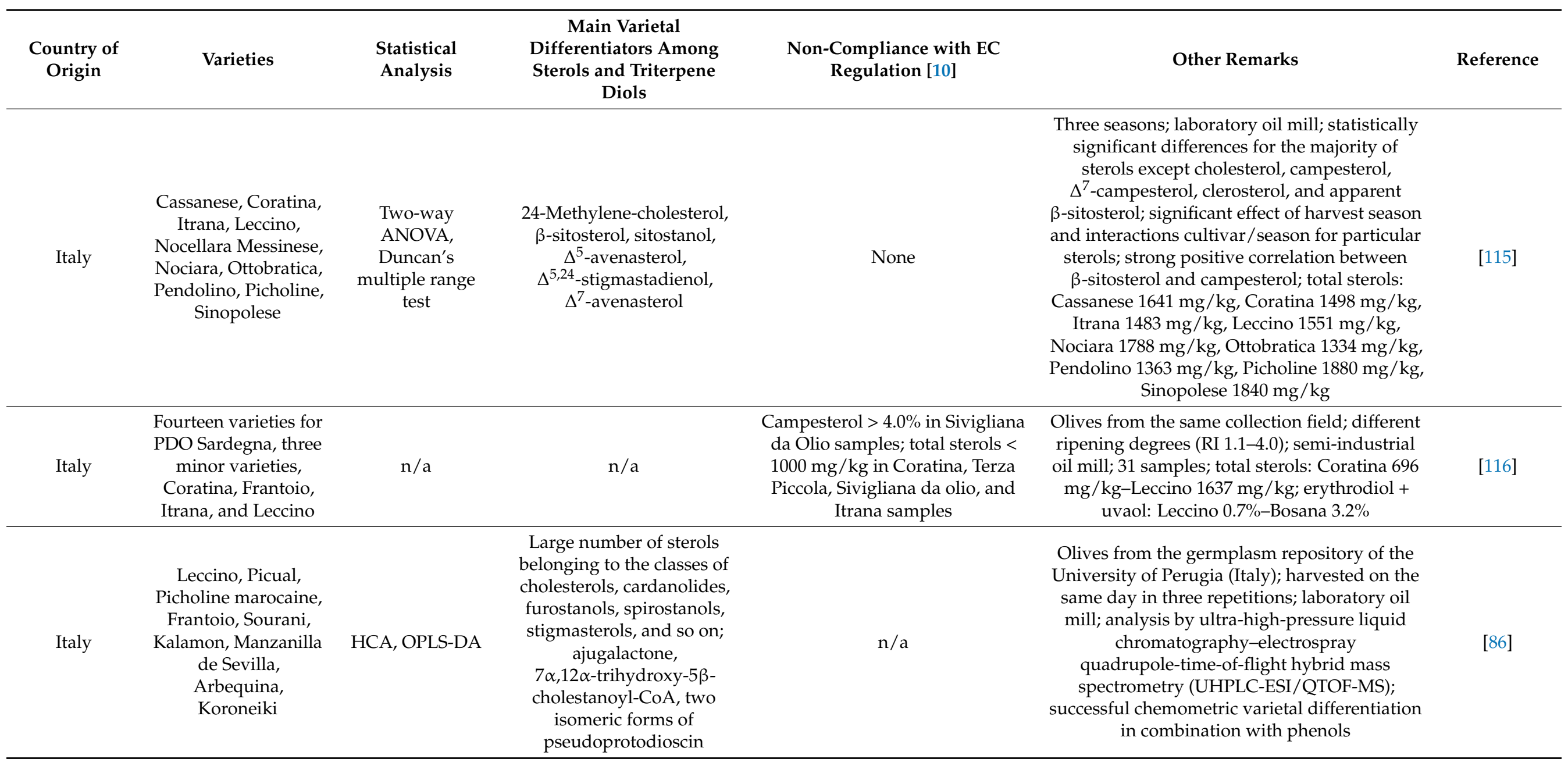


Table 2. Cont.

\begin{tabular}{|c|c|c|c|c|c|c|}
\hline $\begin{array}{l}\text { Country of } \\
\text { Origin }\end{array}$ & Varieties & $\begin{array}{l}\text { Statistical } \\
\text { Analysis }\end{array}$ & $\begin{array}{l}\text { Main Varietal } \\
\text { Differentiators Among } \\
\text { Sterols and Triterpene } \\
\text { Diols }\end{array}$ & $\begin{array}{c}\text { Non-Compliance with EC } \\
\text { Regulation [10] }\end{array}$ & Other Remarks & Reference \\
\hline Greece & Koroneiki & $\mathrm{n} / \mathrm{a}$ & $\mathrm{n} / \mathrm{a}$ & $\begin{array}{c}\text { Campesterol }>4.0 \% \text { in } 21.7 \% \\
\text { samples; total sterols }<1000 \\
\text { mg } / \mathrm{kg} \text { in } 43.5 \% \text { samples; total } \\
\text { sterols }<1100 \mathrm{mg} / \mathrm{kg} \text { (limit for } \\
\text { Kalamata PDO) in } 66.7 \% \\
\text { samples; erythrodiol + uvaol }> \\
4.5 \% \text { in } 8.06 \% \text { samples }\end{array}$ & $\begin{array}{c}\text { Industrial processing in various mills; } 71 \\
\text { samples; total sterols } 1033 \mathrm{mg} / \mathrm{kg} \text { (average), } \\
744-1283 \mathrm{mg} / \mathrm{kg} \text { (range); erythrodiol + uvaol: } \\
2.85 \% \text { (average) }\end{array}$ & [118] \\
\hline Greece & $\begin{array}{l}\text { Koroneiki, } \\
\text { Mastoides }\end{array}$ & ANOVA, PCA & Not specified & $\begin{array}{l}\text { Total sterols < } 1000 \mathrm{mg} / \mathrm{kg} \text { in } \\
\text { particular Koroneiki samples }\end{array}$ & $\begin{array}{l}\text { Each cultivar from a different region; } \\
\text { industrially produced oils; } 112 \text { samples; } \\
\text { statistically significant differences for the } \\
\text { majority of sterols and triterpene diols; } \\
\text { successful chemometric varietal differentiation, } \\
\text { better in combination with FAMEs; total sterols: } \\
\text { Koroneiki } 1033 \mathrm{mg} / \mathrm{kg} \text {, Mastoides } 1220 \mathrm{mg} / \mathrm{kg} \text {; } \\
\text { erythrodiol + uvaol: Koroneiki } 2.85 \% \text {, } \\
\text { Mastoides } 1.40 \%\end{array}$ & [119] \\
\hline Greece & $\begin{array}{l}\text { Lianolia Kerkyras, } \\
\text { Koroneiki }\end{array}$ & ANOVA, PCA & Not specified & $\begin{array}{l}\text { Total sterols < } 1000 \mathrm{mg} / \mathrm{kg} \text { in } \\
40.9 \% \text { Koroneiki samples }\end{array}$ & $\begin{array}{l}\text { Three growing regions; industrial processing in } \\
\text { various mills; } 104 \text { samples; statistically } \\
\text { significant differences for the majority of } \\
\text { sterols and triterpene diols; successful } \\
\text { chemometric varietal differentiation, even } \\
\text { better in combination with FAMEs; total sterols: } \\
\text { Lianolia Kerkyras } 1344 \mathrm{mg} / \mathrm{kg} \text {, Koroneiki } 1021 \\
\mathrm{mg} / \mathrm{kg} \text { (average); erythrodiol + uvaol: Lianolia } \\
\text { Kerkyras } 1.43 \% \text {, Koroneiki } 2.76 \% \text { (average) }\end{array}$ & [120] \\
\hline
\end{tabular}


Table 2. Cont.

\begin{tabular}{|c|c|c|c|c|c|c|}
\hline $\begin{array}{l}\text { Country of } \\
\text { Origin }\end{array}$ & Varieties & $\begin{array}{l}\text { Statistical } \\
\text { Analysis }\end{array}$ & $\begin{array}{c}\text { Main Varietal } \\
\text { Differentiators Among } \\
\text { Sterols and Triterpene } \\
\text { Diols }\end{array}$ & $\begin{array}{l}\text { Non-Compliance with EC } \\
\text { Regulation [10] }\end{array}$ & Other Remarks & Reference \\
\hline Turkey & Ayvalik, Memecik & $\begin{array}{l}\text { ANOVA, PCA, } \\
\text { LDA }\end{array}$ & $\begin{array}{l}\text { Stigmasterol, apparent } \\
\beta \text {-sitosterol, total sterols } \\
\text { followed by campesterol }\end{array}$ & $\begin{array}{c}\text { Campesterol }>4.0 \% \text { in all } \\
\text { Memecik samples }\end{array}$ & $\begin{array}{c}\text { Two seasons; two growing regions; } 16 \\
\text { commercial samples; successful chemometric } \\
\text { varietal differentiation; sterols better } \\
\text { differentiators than phenols and tocopherols; } \\
\text { apparent } \beta \text {-sitosterol and total sterol affected by } \\
\text { season; negative correlation between } \beta \text {-sitosterol } \\
\text { and } \Delta^{5} \text {-avenasterol; Ayvalik relatively high } \\
\Delta^{5} \text {-avenasterol } 17.27-22.17 \% \text {; total sterols: } \\
\text { Ayvalik } 2135-2673 \mathrm{mg} / \mathrm{kg} \text {, Memecik } 1157-1676 \\
\mathrm{mg} / \mathrm{kg}\end{array}$ & [121] \\
\hline Turkey & Eighteen varieties & $\begin{array}{l}\text { ANOVA, } \\
\text { Duncan's } \\
\text { multiple range } \\
\text { test, regression } \\
\text { analysis, PCA, } \\
\text { DA }\end{array}$ & $\begin{array}{c}\text { Cholesterol, campesterol, } \\
\text { stigmasterol, } \\
\Delta^{7} \text {-campesterol, } \beta \text {-sitosterol, } \\
\Delta^{5} \text {-avenasterol, } \\
\Delta^{5,24} \text {-stigmastadienol, } \\
\Delta^{7} \text {-avenasterol }\end{array}$ & $\begin{array}{c}\text { Campesterol }>4.0 \% \text { and } \\
\text { cholesterol }>0.5 \% \text { in Girit } \\
\text { samples; } \Delta^{7} \text {-stigmastenol }>0.5 \% \\
\text { in Erkence, Mersin yaglık, } \\
\text { Saurani, Kilis yaglık, and Sarı } \\
\text { hasebi samples }\end{array}$ & $\begin{array}{l}\text { Two harvest years; different growing regions; } \\
\text { laboratory oil mill; } 101 \text { samples; statistically } \\
\text { significant differences for the majority of sterols } \\
\text { and triterpene diols; for five major varieties } \\
\text { (Gemlik, Memecik, Edremit, Nizip yaglik, Kilis } \\
\text { yaglik), sterols have better chemometric varietal } \\
\text { differentiators than triglycerides and FAMEs; } \\
\text { negative correlation between } \beta \text {-sitosterol and } \\
\Delta^{5} \text {-avenasterol; total sterols: Kalamata } 1145 \\
\text { mg/kg-Edremit yaglik } 2212 \mathrm{mg} / \mathrm{kg} \text {; erythrodiol } \\
+ \text { uvaol: } 0.69-4.42 \%\end{array}$ & [122] \\
\hline Turkey & Gemlik, Halhalı & $\begin{array}{l}\text { ANOVA, } \\
\text { Duncan's } \\
\text { multiple range } \\
\text { test }\end{array}$ & Not specified & $\begin{array}{c}\text { Brassicasterol }>0.1 \% \text { in a } \\
\text { particular Gemlik sample; } \\
\Delta^{7} \text {-stigmastenol }>0.5 \% \text { and } \\
\text { apparent } \beta \text {-sitosterol }<93 \% \text { in } \\
\text { particular Halhalı samples }\end{array}$ & $\begin{array}{c}\text { Four ripening degrees; two growing regions; } \\
\text { laboratory oil mill; } 16 \text { samples; significant } \\
\text { differences for all sterols and triterpene diols; } \\
\text { significant effect of ripening degree and growing } \\
\text { region; negative correlation between } \beta \text {-sitosterol } \\
\text { and } \Delta^{5} \text {-avenasterol; Halhalı rather low } \\
\Delta^{5} \text {-avenastrol } 2.74-3.73 \% \text {; total sterols: Gemlik } \\
1325-2009 \mathrm{mg} / \mathrm{kg} \text {, Halhall } 1194-1569 \mathrm{mg} / \mathrm{kg} ; \\
\text { erythrodiol + uvaol: } 1.16-4.09 \%\end{array}$ & [123] \\
\hline
\end{tabular}


Table 2. Cont.

\begin{tabular}{|c|c|c|c|c|c|c|}
\hline $\begin{array}{l}\text { Country of } \\
\text { Origin }\end{array}$ & Varieties & $\begin{array}{l}\text { Statistical } \\
\text { Analysis }\end{array}$ & $\begin{array}{c}\text { Main Varietal } \\
\text { Differentiators Among } \\
\text { Sterols and Triterpene } \\
\text { Diols }\end{array}$ & $\begin{array}{c}\text { Non-Compliance with EC } \\
\text { Regulation [10] }\end{array}$ & Other Remarks & Reference \\
\hline Turkey & $\begin{array}{l}\text { Sarı Hasebi, } \\
\text { Gemlik, Halhalı }\end{array}$ & $\begin{array}{l}\text { ANOVA, Duncan's } \\
\text { multiple range test, } \\
\text { correlation analysis }\end{array}$ & Not specified & $\begin{array}{l}\Delta^{7} \text {-Stigmastenol }>0.5 \% \text { in all Sari } \\
\text { Hasebi and ripe Halhalı samples; } \\
\text { cholesterol }>0.5 \% \text { in earlier } \\
\text { harvest Sarı Hasebi and Gemlik } \\
\text { samples; apparent } \beta \text {-sitosterol }< \\
93 \% \text { in all Sari Hasebi samples; } \\
\text { total sterols }<1000 \mathrm{mg} / \mathrm{kg} \text { in } \\
\text { earlier harvest all varieties; } \\
\text { erythrodiol + uvaol }>4.5 \% \text { in } \\
\text { early harvest Sari Hasebi samples }\end{array}$ & $\begin{array}{l}\text { Three ripening degrees (RI from } 1.9 \text { to } 4.8 \text { ); } \\
\text { laboratory oil mill; nine samples; statistically } \\
\text { significant differences for the majority of sterols; } \\
\text { significant effect of ripening degree; negative } \\
\text { correlation between } \beta \text {-sitosterol and } \Delta^{5} \text {-avenasterol; } \\
\text { total sterols: Sarı Hasebi } 358-1061 \mathrm{mg} / \mathrm{kg} \text {, Gemlik } \\
728-1001 \mathrm{mg} / \mathrm{kg}, \text { Halhal1 } 898-1092 \mathrm{mg} / \mathrm{kg} \text {; } \\
\text { erythrodiol + uvaol: } 1.78-4.52 \%\end{array}$ & [124] \\
\hline Turkey & $\begin{array}{l}\text { Buyuk Topak } \\
\text { Ulak, Gemlik, } \\
\text { Sari Ulak, } \\
\text { Kargaburun, } \\
\text { Hasebi, Halhali }\end{array}$ & $\begin{array}{l}\text { ANOVA, Duncan's } \\
\text { multiple range test }\end{array}$ & Not specified & $\begin{array}{l}\text { Stigmasterol > campesterol in a } \\
\text { particular Gemlik sample; total } \\
\text { sterols }<1000 \mathrm{mg} / \mathrm{kg} \text { in } \\
\text { particular Kargaburun, Halhali, } \\
\text { and Gemlik samples }\end{array}$ & $\begin{array}{l}\text { Four growing regions; RI from } 1.4 \text { to } 6.3 \text {; laboratory } \\
\text { oil mill; } 24 \text { samples; statistically significant } \\
\text { differences for all sterols and triterpene diols; } \\
\text { significant effect of growing region; negative } \\
\text { correlation between } \beta \text {-sitosterol and } \Delta^{5} \text {-avenasterol; } \\
\Delta^{5} \text {-avenasterol fluctuated notably from } 1.82 \text { to } 18.9 \% \text {; } \\
\text { total sterols: Buyuk Topak Ulak } 4519 \mathrm{mg} / \mathrm{kg} \text {, Gemlik } \\
\quad 880-2057 \mathrm{mg} / \mathrm{kg} \text {, Sari Ulak } 3095 \mathrm{mg} / \mathrm{kg} \text {, } \\
\text { Kargaburun } 720 \mathrm{mg} / \mathrm{kg} \text {, Hasebi } 1804 \mathrm{mg} / \mathrm{kg} \text {, } \\
\text { Halhali } 789 \mathrm{mg} / \mathrm{kg} \text {; erythrodiol + uvaol: } 0.07-4.27 \%\end{array}$ & [125] \\
\hline Tunisia & $\begin{array}{l}\text { Chétoui, Jarboui, } \\
\text { Ain Jarboua, } \\
\text { Neb Jmel, } \\
\text { Rekhami, } \\
\text { Regregui }\end{array}$ & $\begin{array}{l}\text { Duncan's multiple } \\
\text { range test, HCA }\end{array}$ & $\begin{array}{c}\Delta^{5} \text {-Avenasterol, } \\
\text { stigmasterol, total sterols } \\
\text { (Chétoui vs. others); } \\
\beta \text {-sitosterol, apparent } \\
\beta \text {-sitosterol (Regregui } \\
\text { from others) }\end{array}$ & None & $\begin{array}{c}\text { Three seasons; Abencor laboratory oil mill; six } \\
\text { samples; statistically significant differences for all } \\
\text { sterols and triterpene diols; negative correlation } \\
\text { between } \beta \text {-sitosterol and } \Delta^{5} \text {-avenasterol; Regreui: } \\
\text { very low } \Delta^{5} \text {-avenasterol of } 3.79 \% \text {; total sterols: } \\
\text { Chétoui } 1288 \mathrm{mg} / \mathrm{kg} \text {, Jarboui } 1964 \mathrm{mg} / \mathrm{kg} \text {, Ain } \\
\text { Jarboua } 1600 \mathrm{mg} / \mathrm{kg} \text {, Neb Jmel } 2048 \mathrm{mg} / \mathrm{kg} \text {, } \\
\text { Rekhami } 1662 \mathrm{mg} / \mathrm{kg} \text {, Regregui } 2292 \mathrm{mg} / \mathrm{kg} \text {; } \\
\text { erythrodiol + uvaol } 1.38-2.60 \%\end{array}$ & [126] \\
\hline
\end{tabular}


Table 2. Cont.

\begin{tabular}{|c|c|c|c|c|c|c|}
\hline $\begin{array}{l}\text { Country of } \\
\text { Origin }\end{array}$ & Varieties & $\begin{array}{l}\text { Statistical } \\
\text { Analysis }\end{array}$ & $\begin{array}{c}\text { Main Varietal } \\
\text { Differentiators Among } \\
\text { Sterols and Triterpene } \\
\text { Diols }\end{array}$ & $\begin{array}{c}\text { Non-Compliance with EC } \\
\text { Regulation [10] }\end{array}$ & Other Remarks & Reference \\
\hline Tunisia & $\begin{array}{c}\text { Chemlali } \\
\text { Tataouine, Fakhari } \\
\text { Douirat, Zarrazi } \\
\text { Douirat }\end{array}$ & $\begin{array}{l}\text { ANOVA, Duncan's } \\
\text { multiple range test }\end{array}$ & Not specified & None & $\begin{array}{l}\text { Similar RI; Abencor laboratory oil mill; nine } \\
\text { samples; statistically significant differences for } \\
\text { the majority of sterols and triterpene diols } \\
\text { except for campestanol or stigmasterol; } \\
\text { negative correlation between } \beta \text {-sitosterol and } \\
\Delta^{5} \text {-avenasterol; total sterols: Chemlali } \\
\text { Tataouine } 1717 \mathrm{mg} / \mathrm{kg} \text {, Fakhari Douirat } 1041 \\
\mathrm{mg} / \mathrm{kg} \text {, Zarrazi Douirat } 1063 \mathrm{mg} / \mathrm{kg} ; \\
\text { erythrodiol + uvaol: } 1.5-2.4 \%\end{array}$ & [127] \\
\hline Tunisia & $\begin{array}{l}\text { Semni, Jdallou, } \\
\text { Chemlali Sfax, } \\
\text { Swabâa Algia, } \\
\text { Oueslati, El Hor }\end{array}$ & ANOVA, HCA & Not specified & $\begin{array}{c}\text { Brassicasterol }>0.1 \% \text { in Semni } \\
\text { samples; } \Delta^{7} \text {-stigmastenol }>0.5 \% \\
\text { in all samples except Jdallou and } \\
\text { Chemlali Sfax; apparent } \\
\beta \text {-sitosterol }<93 \% \text { in Oueslati } \\
\text { samples }\end{array}$ & $\begin{array}{l}\text { RI between } 4 \text { and } 5.5 \text {; Abencor laboratory oil } \\
\text { mill; statistically significant differences for the } \\
\text { majority of major and some minor sterols; } \\
\text { successful chemometric varietal differentiation } \\
\text { in combination with aliphatic and triterpenic } \\
\text { alcohols, FAMEs, volatiles, and basic quality } \\
\text { parameters; very high } \Delta^{5} \text {-avenasterol } \\
\text { proportion in El Hor samples of } 31 \% \text {; total } \\
\text { sterols: Semni } 1390 \mathrm{mg} / \mathrm{kg} \text {, Jdallou } 2320 \\
\mathrm{mg} / \mathrm{kg} \text {, Chemlali Sfax } 1926 \mathrm{mg} / \mathrm{kg} \text {, Swabâa } \\
\text { Algia } 1488 \mathrm{mg} / \mathrm{kg} \text {, Oueslati } 1508 \mathrm{mg} / \mathrm{kg}, \mathrm{El} \\
\text { Hor } 1173 \mathrm{mg} / \mathrm{kg} \text {; erythrodiol + uvaol: } \\
1.08-2.79 \%\end{array}$ & [128] \\
\hline Tunisia & $\begin{array}{c}\text { Hor Kesra, Sredki, } \\
\text { Chladmi, Betsijina, } \\
\text { Aloui }\end{array}$ & $\begin{array}{l}\text { ANOVA, Duncan's } \\
\text { multiple range test, } \\
\text { PCA, HCA }\end{array}$ & $\begin{array}{c}\Delta^{7} \text {-Stigmastenol, } \\
\text { 24-methylene-cholesterol, } \\
\text { clerosterol, } \beta \text {-sitosterol }\end{array}$ & None & $\begin{array}{l}\text { RI around 3.5; Abencor laboratory oil mill; } 15 \\
\text { samples; statistically significant differences for } \\
\text { the majority of sterols and triterpene diols; } \\
\text { successful chemometric varietal differentiation } \\
\text { in combination with phenols, FAMEs, and } \\
\text { tryglycerides; Hor Kesra } 1039 \mathrm{mg} / \mathrm{kg} \text {, Sredki } \\
1381 \mathrm{mg} / \mathrm{kg} \text {, Chladmi } 1567 \mathrm{mg} / \mathrm{kg} \text {, Betsijina } \\
1382 \mathrm{mg} / \mathrm{kg} \text {, Aloui } 1268 \mathrm{mg} / \mathrm{kg} \text {; erythrodiol + } \\
\text { uval: } 19-32 \mathrm{mg} / \mathrm{kg}\end{array}$ & [129] \\
\hline
\end{tabular}


Table 2. Cont.

\begin{tabular}{|c|c|c|c|c|c|c|}
\hline $\begin{array}{l}\text { Country of } \\
\text { Origin }\end{array}$ & Varieties & $\begin{array}{l}\text { Statistical } \\
\text { Analysis }\end{array}$ & $\begin{array}{c}\text { Main Varietal } \\
\text { Differentiators Among } \\
\text { Sterols and Triterpene } \\
\text { Diols }\end{array}$ & $\begin{array}{c}\text { Non-Compliance with EC } \\
\text { Regulation [10] }\end{array}$ & Other Remarks & Reference \\
\hline Portugal & $\begin{array}{l}\text { Cobrançosa, } \\
\text { Madural, Verdeal } \\
\text { Transmontana }\end{array}$ & Not specified & $\mathrm{n} / \mathrm{a}$ & $\begin{array}{c}\text { Cholesterol }>0.5 \% \text { in particular } \\
\text { samples }\end{array}$ & $\begin{array}{l}\text { Two ripening degrees; Abencor laboratory oil } \\
\text { mill (six samples) and commercial oils (nine } \\
\text { samples); free and esterified sterols analyzed } \\
\text { separately; quantitative dominance of free } \\
\text { sterols; total sterols: } 1046-1829 \mathrm{mg} / \mathrm{kg}\end{array}$ & [95] \\
\hline Portugal & $\begin{array}{l}\text { Cobrançosa, } \\
\text { Madural, Verdeal } \\
\text { Transmontana }\end{array}$ & $\begin{array}{l}\text { MANOVA, PCA, } \\
\text { CA }\end{array}$ & $\begin{array}{c}\Delta^{5} \text {-Avenasterol, } \\
\text { stigmasterol, } \Delta^{7} \text {-avenasterol }\end{array}$ & None & $\begin{array}{l}\text { Abencor laboratory oil mill; } 18 \text { samples; } \\
\text { successful chemometric varietal differentiation } \\
\text { despite different ripening degrees, better in } \\
\text { combination with tocopherols; total sterols: } \\
\text { Cobrançosa } 1654-2678 \mathrm{mg} / \mathrm{kg} \text {, Madural } \\
\text { 1780-2936 mg/kg, Verdeal Transmontana } \\
\text { 1365-2183 mg/kg }\end{array}$ & [131] \\
\hline Algeria & $\begin{array}{c}\text { Chemlal, } \\
\text { Aghenfas, } \\
\text { Buichret, Mekki }\end{array}$ & $\begin{array}{l}\text { ANOVA, PCA, } \\
\text { LDA }\end{array}$ & $\begin{array}{c}\text { Cholesterol, campestanol, } \\
\Delta^{7} \text {-stigmastenol, } \\
\Delta^{7} \text {-avenasterol, clerosterol }\end{array}$ & None & $\begin{array}{c}\text { Eight ripening degrees; Abencor laboratory oil } \\
\text { mill; } 32 \text { samples; significant differences for all } \\
\text { sterols and triterpene diols; significant effect of } \\
\text { ripening degree; negative correlation between } \\
\beta \text {-sitosterol and } \Delta^{5} \text {-avenasterol; relatively } \\
\text { successful chemometric varietal differentiation in } \\
\text { combination with aliphatic alcohols; total sterols: } \\
\text { Chemlal } 1745-2170 \mathrm{mg} / \mathrm{kg} \text {, Aghenfas } 1882-2304 \\
\mathrm{mg} / \mathrm{kg} \text {, Buichret } 1187-1664 \mathrm{mg} / \mathrm{kg} \text {, Mekki } \\
1748-1985 \mathrm{mg} / \mathrm{kg} \text {; erythrodiol + uvaol: } 18-58 \\
\mathrm{mg} / \mathrm{kg}\end{array}$ & [132] \\
\hline
\end{tabular}


Table 2. Cont.

\begin{tabular}{|c|c|c|c|c|c|c|}
\hline $\begin{array}{l}\text { Country of } \\
\text { Origin }\end{array}$ & Varieties & $\begin{array}{l}\text { Statistical } \\
\text { Analysis }\end{array}$ & $\begin{array}{c}\text { Main Varietal } \\
\text { Differentiators Among } \\
\text { Sterols and Triterpene } \\
\text { Diols }\end{array}$ & $\begin{array}{c}\text { Non-Compliance with EC } \\
\text { Regulation [10] }\end{array}$ & Other Remarks & Reference \\
\hline Argentina & $\begin{array}{c}\text { Arbequina, Barnea, } \\
\text { Picual, Frantoio, } \\
\text { Empeltre, Manzanilla, } \\
\text { Arauco, Coratina }\end{array}$ & $\begin{array}{c}\text { ANOVA, } \\
\text { Student's } t \text {-test, } \\
\text { PCA, CA }\end{array}$ & $\begin{array}{l}\text { Apparent } \beta \text {-sitosterol, } \\
\text { total sterols }\end{array}$ & $\begin{array}{l}\text { Cholesterol }>0.5 \% \text { in particular } \\
\text { Arbequina, Arauco, and Coratina } \\
\text { samples; brassicasterol }>0.1 \% \text { in } \\
\text { an Arbequina and an Arauco } \\
\text { sample; campesterol }>4.0 \% \text { in } 70 \% \\
\text { Arbequina, all Barnea, and a single } \\
\text { Arauco sample; } \Delta^{7} \text {-stigmastenol }> \\
0.5 \% \text { in a Coratina sample; } \\
\text { apparent } \beta \text {-sitosterol }<93 \% \text { in } \\
\text { particular Arbequina and in } \\
\text { Frantoio and Arauco samples }\end{array}$ & $\begin{array}{l}\text { Two seasons; different growing regions; RI } \\
1.3-4.8 \text {; industrial processing in various mills; } \\
37 \text { samples; relatively successful chemometric } \\
\text { varietal differentiation in combination with } \\
\text { other parameters; total sterols: Arbequina } \\
1424-3004 \mathrm{mg} / \mathrm{kg} \text {, other varieties } 1053-2509 \\
\mathrm{mg} / \mathrm{kg} \text {; erythrodiol + uvaol: Arbequina } \\
0.9-1.9 \% \text {, other varieties } 0.5-3.0 \%\end{array}$ & [133] \\
\hline Australia & $\begin{array}{l}\text { Arbequina, Barnea, } \\
\text { Coratina, Corregiolla, } \\
\text { Frantoio, Koreneiki, } \\
\text { Leccino, Manzanillo, } \\
\text { Pendolino, Picual }\end{array}$ & ANOVA & Not specified & $\begin{array}{c}\text { Campesterol }>4.0 \% \text { in particular } \\
\text { Barnea samples from all locations } \\
\text { and particular Koroneiki samples; } \\
\text { total sterols }<1000 \mathrm{mg} / \mathrm{kg} \text { in } \\
\text { particular Koroneiki and } \\
\text { Pendolino samples }\end{array}$ & $\begin{array}{c}\text { Two seasons; four growing regions; Abencor } \\
\text { laboratory oil mill; } 40 \text { samples; statistically } \\
\text { significant effect of variety for all sterols and } \\
\text { triterpene diols, much stronger than the effects } \\
\text { of season and ripening degree; growing region } \\
\text { had a significant effect for many sterols; total } \\
\text { sterols: Arbequina } 1381-2140 \mathrm{mg} / \mathrm{kg} \text {, Barnea } \\
1576-1762 \mathrm{mg} / \mathrm{kg} \text {, Coratina } 1190-1452 \mathrm{mg} / \mathrm{kg} \text {, } \\
\text { Corregiolla } 1159-1812 \mathrm{mg} / \mathrm{kg} \text {, Frantoio } \\
1200-1632 \mathrm{mg} / \mathrm{kg} \text {, Koreneiki 798-1267 mg } / \mathrm{kg} \text {, } \\
\text { Leccino } 1371-1715 \mathrm{mg} / \mathrm{kg} \text {, Manzanillo } \\
1483-1803 \mathrm{mg} / \mathrm{kg}, \text { Pendolino } 944-1183 \mathrm{mg} / \mathrm{kg} \text {, } \\
\text { Picual } 1281-1937 \mathrm{mg} / \mathrm{kg} \text {; erythrodiol + uvaol: } \\
0.45-4.0 \%\end{array}$ & [134] \\
\hline USA & $\begin{array}{l}\text { Carolea, Casaliva, } \\
\text { Cayon, Frantoio, } \\
\text { Kalamon, Maurino, } \\
\text { Moraiolo, Taggiasca }\end{array}$ & $\begin{array}{l}\text { ANOVA, PCA, } \\
\text { PLS-DA }\end{array}$ & $\begin{array}{c}\beta \text {-Sitosterol, } \\
\Delta^{5} \text {-avenasterol }\end{array}$ & $\mathrm{n} / \mathrm{a}$ & $\begin{array}{l}\text { Experimental orchard; RI 2.3-2.9; Abencor } \\
\text { laboratory oil mill; } 32 \text { samples; successful } \\
\text { chemometric varietal differentiation in } \\
\text { combination with phenols, tocopherols, } \\
\text { triterpenic alcohols, and FAMEs }\end{array}$ & [135] \\
\hline
\end{tabular}


Table 2. Cont.

\begin{tabular}{|c|c|c|c|c|c|c|}
\hline $\begin{array}{c}\text { Country of } \\
\text { Origin }\end{array}$ & Varieties & $\begin{array}{c}\text { Statistical } \\
\text { Analysis }\end{array}$ & $\begin{array}{c}\text { Main Varietal } \\
\text { Differentiators Among } \\
\text { Sterols and Triterpene } \\
\text { Diols }\end{array}$ & $\begin{array}{l}\text { Non-Compliance with EC } \\
\text { Regulation [10] }\end{array}$ & Other Remarks & Reference \\
\hline Iran & $\begin{array}{l}\text { Beleydi, Mission, } \\
\text { Koroneiki }\end{array}$ & ANOVA, PCA & $\begin{array}{l}\beta \text {-Sitosterol, } \Delta^{5} \text {-avenasterol, } \\
\text { campesterol, clerosterol, } \\
\text { stigmasterol, cholesterol }\end{array}$ & $\begin{array}{c}\text { Apparent } \beta \text {-sitosterol }<93 \% \text { and } \\
\Delta^{7} \text {-stigmastenol }>0.5 \% \text { in } \\
\text { particular Koroneiki and Mission } \\
\text { samples }\end{array}$ & $\begin{array}{c}\text { Two growing regions; RI 5; Abencor laboratory } \\
\text { oil mill; six samples; statistically significant } \\
\text { differences for all sterols; significant effect of } \\
\text { growing region; relatively successful } \\
\text { chemometric varietal differentiation; negative } \\
\text { correlation between } \beta \text {-sitosterol and } \\
\Delta^{5} \text {-avenasterol; total sterols: Beleydi } 1609-1778 \\
\text { mg } / \mathrm{kg} \text {, Mission } 1520-1785 \mathrm{mg} / \mathrm{kg} \text {, Koroneiki } \\
1354-1756 \mathrm{mg} / \mathrm{kg}\end{array}$ & [136] \\
\hline Croatia & $\begin{array}{l}\text { Buža, Črna, } \\
\text { Rosinjola }\end{array}$ & $\begin{array}{l}\text { MANOVA, PCA, } \\
\text { SLDA }\end{array}$ & $\begin{array}{c}\text { Campesterol, } \beta \text {-sitosterol, } \\
\Delta^{7} \text {-campesterol } / \Delta^{5,24} \text { - } \\
\text { stigmastadienol, clerosterol, } \\
\text { uvaol, campestanol/ } \Delta^{7} \text { - } \\
\text { avenasterol (fresh oils), later } \\
\text { three substituted by } \\
\text { 24-methylene- } \\
\text { cholesterol/stigmasterol } \\
\text { (aged oils) }\end{array}$ & $\begin{array}{c}\Delta^{7} \text {-Stigmastenol }>0.5 \% \text { and } \\
\text { apparent } \beta \text {-sitosterol }<93 \% \text { in } \\
\text { particular Rosinjola samples }\end{array}$ & $\begin{array}{l}\text { Three ripening degrees approximately RI 1.5-2, } \\
\text { 3, 4; industrial olive processing; } 36 \text { samples; } \\
\text { relatively successful chemometric varietal } \\
\text { differentiation of fresh and aged oils } \\
\text { independently from ripening degree and vice } \\
\text { versa; total sterols: Buža } 1178-1305 \mathrm{mg} / \mathrm{kg} \text {, } \\
\text { Črna } 2078-2277 \mathrm{mg} / \mathrm{kg} \text {, Rosinjola } 1483-1813 \\
\mathrm{mg} / \mathrm{kg} \text {; erythrodiol + uvaol: } 0.78-2.28 \%\end{array}$ & [137] \\
\hline Croatia & $\begin{array}{l}\text { Bova, Buža } \\
\text { puntoža, Istarska } \\
\text { bjelica, Buža, } \\
\text { Rosinjola }\end{array}$ & $\begin{array}{l}\text { ANOVA, Tukey's } \\
\text { honest test }\end{array}$ & Not specified & None & $\begin{array}{c}\text { Same orchard; RI 2.5-3.0, except late ripening } \\
\text { Istarska bjelica RI 1.1; statistically significant } \\
\text { differences for all sterols and triterpene diols; } \\
\text { high } \Delta^{5} \text {-avenasterol in Istarska bjelica } 26.6 \% \text {; } \\
\text { Bova } 2964 \mathrm{mg} / \mathrm{kg} \text {, Buža puntoža } 2158 \mathrm{mg} / \mathrm{kg} \text {, } \\
\text { Istarska bjelica } 1451 \mathrm{mg} / \mathrm{kg} \text {, Buža } 1567 \mathrm{mg} / \mathrm{kg} \text {, } \\
\text { Rosinjola } 1648 \mathrm{mg} / \mathrm{kg} \text {; erythrodiol + uvaol: } \\
0.53-3.16 \%\end{array}$ & [138] \\
\hline
\end{tabular}

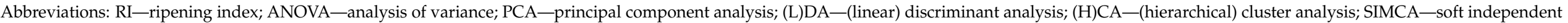

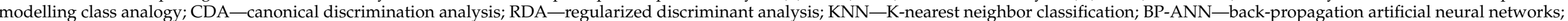
OPLS-DA—orthogonal partial least squares discriminant analysis; CVA—canonical variates analysis; MANOVA—-multivariate analysis of variance. 
A large number of varieties were studied, including the economically and traditionally most important, as well as minor ones. The most abundant sterols in all the investigated olive oils were, as expected, $\beta$-sitosterol, followed by $\Delta^{5}$-avenasterol, campesterol, and stigmasterol. Great variability in sterol and triterpene diol content and composition was found (Table 2). For example, the percentage of one of the most important olive oil sterols, $\Delta^{5}$-avenasterol, ranged from $1.82 \%$ in Buyuk Topak Ulak [125] to $31 \%$ in El Hor monovarietal oil [128]. The content of total sterols also ranged widely from values far below the regulatory limit of $1000 \mathrm{mg} / \mathrm{kg}$ [10], such as $358 \mathrm{mg} / \mathrm{kg}$ in Sar1 Hasebi [124], to more than $4500 \mathrm{mg} / \mathrm{kg}$ in Buyuk Topak Ulak monovarietal oil [125], with an exception reporting extreme concentrations as high as more than $7000 \mathrm{mg} / \mathrm{kg}$ [114]. The majority of the studied oils had total sterols' concentration ranging from 1000 to $3000 \mathrm{mg} / \mathrm{kg}$ (Table 2). Kyçyk et al. [108] proposed the following classification of virgin olive oils based on total sterol content: category I: very high (>2200 mg/kg), category II: high (1999-2200 mg/kg), category III: medium (1650-1999 mg/kg), category IV: low (1300-1650 mg/kg), and category $\mathrm{V}$ : very low $(<1300 \mathrm{mg} / \mathrm{kg})$. Triterpene diols also showed great variation, with erythrodiol + uvaol percentage ranging from 0.0 to $6.1 \%$ [85]. Because, in a study of Allouche et al. [109], erythrodiol was found responsible for $96 \%$ of variance in differentiating 40 monovarietal virgin olive oils, the authors suggested olive oil classification based solely on its content and neglecting uvaol in the following manner: group I: very high content, group II: high content, group III: intermediate content, group IV: intermediate to low content, and group $\mathrm{V}$ : low content of erythrodiol.

A strong negative correlation between the two most prominent sterols, $\beta$-sitosterol and $\Delta^{5}$-avenasterol, was noted in a very large number of studies: particular varieties yielded oils with high $\beta$-sitosterol and low $\Delta^{5}$-avenasterol proportion, and others vice versa (Table 2 ). It is known that a period in olive development is often characterized by a decrease in $\beta$-sitosterol and an increase in $\Delta^{5}$-avenasterol percentage $[52,117,139,140]$. Aparicio and Luna [140] proposed that a cause for this could be the reduction in the proportion of stone in olive weight during ripening, as oil from the stone contains a much higher proportion of $\beta$ sitosterol in relation to $\Delta^{5}$-avenasterol than oil from the pulp [111]. Oils obtained from olive fruits of smaller size, and possibly with a greater stone/flash ratio, were found to have more $\beta$-sitosterol and less $\Delta^{5}$-avenasterol than oils from larger size fruits of the same variety [13]. Based on such premises, a possibility that varieties with a higher proportion of stone in fruit yield oils with a higher $\beta$-sitosterol $/ \Delta^{5}$-avenasterol ratio should not be excluded. Another probable cause derives from the fact that $\Delta^{5}$-avenasterol is a precursor in the biosynthesis of $\beta$-sitosterol [15] and that different enzymatic activity of, e.g., $\Delta^{24}$-isomerase/reductase (Figure 2), predetermined by the genotype, may result in differences in the accumulation of these sterols. On the other hand, Gutiérrez et al. [52] suggested the activity of a particular desaturase enzyme, which transforms $\beta$-sitosterol into $\Delta^{5}$-avenasterol. Kyçyk et al. [108] differentiated virgin olive oils from 43 varieties from the World Olive Germplasm Bank into five groups based on $\beta$-sitosterol and $\Delta^{5}$-avenasterol proportions.

Based on the data from previous studies summarized in Table 2, it can be concluded with maximum certainty that variety has a massive impact on the content and composition of sterols and triterpene diols in virgin olive oil. In the vast majority of such studies, the concentrations and/or relative amounts in different monovarietal oils were statistically compared by analysis of variance (ANOVA) and various post-hoc tests (Table 2). In most cases, significant differences between varieties were found for each particular sterol and triterpene diol. In addition, in a large number of studies multivariate statistical techniques, mostly principal component analysis (PCA), linear discriminant analysis (LDA), and hierarchical clustering analysis (HCA), as well as others, were applied to find combinations of variables and build models that allow the best possible varietal differentiation (Table 2). It should be noted that the reported studies differed greatly in experimental design, including the number of varieties compared, type of samples included (experimentally or industrially produced and commercial oils) and their number, interaction with other factors (growing season or region, ripening degree, growing or processing parameters, 
and so on), combination with other olive oil physico-chemical parameters, and so on. From this aspect, it is necessary to take caution when concluding and comparing their varietal differentiation performance.

Among sterols, those that exhibited the most significant varietal differentiation potential were $\beta$-sitosterol (in 16 studies listed in Table 2 ), campesterol (14 studies), $\Delta^{5}$-avenasterol (12 studies), and stigmasterol (10 studies), while $\Delta^{7}$-avenasterol (7 studies), clerosterol (6 studies), $\Delta^{5,24}$-stigmastadienol (5 studies), and $\Delta^{7}$-stigmastenol (5 studies) were also useful in several cases. Other sterols and triterpene diols stood out sporadically. It is interesting to mention a study in which the authors applied untargeted metabolomics approach by ultra-high-pressure liquid chromatography coupled to an electrospray quadrupoletime-of-flight hybrid mass spectrometer (UHPLC-ESI/QTOF-MS) and identified a much larger number of sterols than is possible using the standard method. In this case, a large number of minor, some of them previously unidentified, sterols showed varietal discrimination potential by chemometric analyses, with unfamiliar sterols, such as ajugalactone, $7 \alpha, 12 \alpha$-trihydroxy- $5 \beta$-cholestanoyl-CoA, and two isomeric forms of pseudoprotodioscin, emerging as the most useful differentiators [86].

It must be noted that, in the studies reported in Table 2, the multivariate models developed for varietal differentiation were in many cases more successful or successful only when including other olive oil parameters in combination with sterols $[71,98,100,105-107$, $110,112,113,119,120,128,129,132,133,135]$, while successful models that used only sterol and triterpene diol compositional data were fewer in number $[82,85,99,101,111,120,130,131,137]$. However, studies were published in which sterols served as more important chemometric differentiators than phenols and tocopherols [121] or triglycerides and FAMEs [122]. It was shown that the effect of variety may interact with other factors, such as growing season $[102,104,105,115,121]$, ripening degree $[104,117,123,124,132]$, and growing region $[74,123,125,136]$. In a study with seven Spanish varieties and five growing regions, in a direct comparison, variety had a stronger influence (expressed as percentage of variance) than geographical location on $\beta$-sitosterol, $\Delta^{5}$-avenasterol, and total sterols [74]. There are also studies that succeeded in developing robust chemometric models in which varietal differentiation using only sterol and triterpene diol compositional data was not compromised by interfering factors, such as ripening degree $[99,131,137]$, growing season $[82,85]$, growing region $[82,85,120]$, or oil age [137]. In some cases, to achieve better varietal differentiation solely by sterols and triterpene diols, variables used were not just their concentrations and proportions, but also their ratios [137].

In virgin olive oils produced from a number of olive varieties, relative amounts of particular sterols and/or triterpenes diols were found to be higher or lower than the limits prescribed by the current regulation (Table 2). For particular Spanish Cornicabra $[98,141]$ and Benizal monovarietal oil samples [102,105], a campesterol content higher than $4.0 \%$ was found in several studies, suggesting it as a possible varietal peculiarity. A similar case was found in particular samples of Greek Koroneiki [118] and Turkish Memecik [121], and in a whole series of oils from minor varieties in various Mediterranean countries (Table 2). Among varieties cultivated outside the Mediterranean basin, Barnea from both Australia [134] and Argentina [133] exhibited the same characteristic. Among several varieties that yielded oils with an insufficient concentration of total sterols, a number of Koroneiki samples obtained in Greece [110,119,120] and Australia [134] stood out. It may seem that, in the other studied monovarietal virgin olive oils, such and other deviations from the regulatory limits occurred mostly sporadically (Table 2). However, given the limited literature data on the majority of the studied monovarietal virgin olive oils, and in fact a relatively high frequency of the occurrence of non-compliant results for sterols and triterpene diols for particular varieties, further research is needed to determine whether these are varietal characteristics or random deviations. It is assumed that the adaptation of olives to new growing areas, such as the USA, Australia, and Argentina [142]; the trend of very early harvesting dates in some areas; and the global problem of climate change are among the possible causes of such 'anomalies'. 
The olive sterol biosynthetic pathway is regulated by a series of enzymes, whose activity is certainly strongly regulated by variety [15]. For example, as already mentioned, the difference in the content and proportion of $\beta$-sitosterol and $\Delta^{5}$-avenasterol and their ratio might be a direct consequence of the expression or enzymatic load of $\Delta^{24}$-isomerase/reductase (Figure 2). In one of the previous steps of the sterol biosynthetic pathway, bifurcation occurs, leading to the formation of $\beta$-sitosterol and stigmasterol (24-ethylsterols) or campesterol (24-methylsterol) from a common precursor gramisterol (24-methylene lophenol), regulated by the activity of two enzymes, SAM-24methylene-lophenol-C-24-methyltransferase2 (SMT2) and C- $4 \alpha$-sterol-methyl-oxidase2 (SMO2) (Figure 2). It is probable that the abovementioned increased campesterol content in olives and oil from particular varieties is a consequence of the stronger expression of $\mathrm{SMO} 2$ and biosynthesis of 24-methyl(ene) sterols and/or, even more probably, weaker expression of SMT2 and biosynthesis of 24-ethyl(idene) sterols. Furthermore, the differences in total sterols between oils from different olive varieties are possibly predetermined by the activity and load of other enzymes, for example, squalene synthase, which regulates the formation of a common sterol precursor squalene in competition with sesquiterpene cyclase, which diverts the synthesis path towards sesquiterpenes [8]. The importance of particular enzymes in sterol biosynthesis has been proven empirically in other plants. A fivefold increase in the activity of 3-hydroxy-3-methylglutaryl coenzyme A reductase (HMGR) was followed by a 15-fold accumulation of free and esterified sterols, while on the other hand, the reduction in SMO1 gene expression accumulated 24-methylene-cycloartanol and the reduction in $\mathrm{SMO} 2$ gene expression produced more citrostadienol at the expense of the synthesis of sterol end-products [15]. The activity of enzymes can be partially conditioned by the availability of substrates, as well as factors other than variety that may interfere [8].

\subsection{Influence of Ripening Degree}

An attempt to summarize the currently available information about the evolution of sterols and triterpene diols during olive fruit ripening and their contents in oils produced therefrom is presented in Table 3.

Ripening of olive fruits can be observed from the outside as an increase in its size, weight, pulp/stone ratio, and change in color. Oil is accumulated through the major part of olive ripening, but its formation stops at a certain point, after which its content often continues to increase at the expense of loss in moisture. Simultaneously with the biosynthesis of triglycerides, the synthesis of other olive phytochemicals takes place, including pigments responsible for a change in color, the precursors of phenols and volatiles crucial for the taste and aroma of olive oil, as well as sterols and triterpene diols, all catalyzed by multienzyme complexes coded by genes $[143,144]$. 
Table 3. Evolution of sterols and triterpene diols during ripening of olives from different varieties based on their levels in olive oils produced therefrom.

\begin{tabular}{|c|c|c|}
\hline \multirow[t]{2}{*}{ Sterol/Triterpene Diol } & \multicolumn{2}{|c|}{ Evolution during Ripening: Variety } \\
\hline & First Phase (RI 0-3 or Analogous) & Second Phase (RI > 3 or Analogous) \\
\hline Cholesterol & $\begin{array}{c}\text { Increase: Halhalı [123]; Memecik, Ayvalik [145]; } \\
\text { relatively constant: Koroneiki [12]; averaged [99]; Gemlik [123]; Aghenfas, } \\
\text { Buichret, Mekki [132]; Buža, Črna, Rosinjola [137]; averaged [146]; Chemlali [147]; } \\
\text { Castellana [148]; Ogliarola garganica [149]; Edremit [150]; } \\
\text { variable: Cobrançosa, Verdeal Transmontana [131]; Chemlal [132]; } \\
\text { mostly decrease: Oueslati [151]; } \\
\text { decrease: averaged [13]; Sarı Hasebi, Gemlik, Halhalı [124]; Memecik [150] }\end{array}$ & $\begin{array}{c}\text { Increase: Ayvalik [145]; } \\
\text { mostly increase: Aghenfas [132]; } \\
\text { relatively constant: averaged [13]; averaged [99]; Halhalı [124]; Chemlal, } \\
\text { Buichret [132]; Buža, Črna, Rosinjola [137]; averaged [146]; Castellana [148]; } \\
\text { Ogliarola garganica [149]; Memecik, Edremit [150]; } \\
\text { variable: Cobrançosa, Madural, Verdeal Transmontana [131]; Mekki [132]; } \\
\text { Chemlali [147]; } \\
\text { decrease: Koroneiki [12]; Halhalı [123]; Sarı Hasebi, Gemlik [124]; Memecik [145]; } \\
\text { Oueslati [151] }\end{array}$ \\
\hline Brassicasterol & $\begin{array}{c}\text { Relatively constant: Halhalı [123]; Sarı Hasebi, Gemlik, Halhalı [124]; Memecik, } \\
\text { Edremit [150]; } \\
\text { decrease: Gemlik [123] }\end{array}$ & $\begin{array}{l}\text { Increase: Sarı Hasebi, Gemlik, Halhalı [124]; Memecik [150]; } \\
\text { relatively constant: Gemlik, Halhalı [123]; Edremit [150] }\end{array}$ \\
\hline 24-Methylenecholesterol & $\begin{array}{c}\text { Increase: Koroneiki [12]; averaged [13]; averaged [99]; Gemlik [124]; Buža, Črna, } \\
\text { Rosinjola [137]; } \\
\text { relatively constant: Gemlik [123]; Halhalı [124]; averaged [146]; Chemlali [147]; } \\
\text { Ogliarola garganica [149]; Memecik, Edremit [150]; } \\
\text { variable: Halhalı [123]; Oueslati [151]; } \\
\text { decrease: Sarı Hasebi [124]; Memecik, Ayvalik [145] }\end{array}$ & $\begin{array}{l}\text { Increase: averaged [99]; Gemlik [124]; Črna [137]; Memecik, Ayvalik [145]; } \\
\text { relatively constant: averaged [13]; Gemlik [123]; Sarı Hasebi, Halhalı [124]; } \\
\text { Rosinjola [137]; averaged [146]; Chemlali [147]; Ogliarola garganica [149]; } \\
\text { Memecik, Edremit [150]; } \\
\text { variable: Halhalı [123]; Oueslati [151]; } \\
\text { decrease: Koroneiki [12]; Buža [137] }\end{array}$ \\
\hline Campesterol & $\begin{array}{c}\text { Increase: Throumbolia [117]; Chemlal [132]; Buža, Črna, Rosinjola [137]; } \\
\text { relatively constant: averaged [13]; Picual, Hojiblanca [52]; averaged [99]; } \\
\text { Koroneiki [117]; Halhalı [123]; Sarı Hasebi [124]; averaged [146]; Castellana [148]; } \\
\text { Ogliarola garganica [149]; Memecik [150]; Chemlali [152]; } \\
\text { variable: Gemlik [123]; Cobrançosa [131]; Buichret [132]; Picholine [139]; } \\
\text { Chemlali [147]; Edremit [150]; Koroneiki [153]; } \\
\text { mostly decrease: Chemlal, Aghenfas, Mekki [132]; Oueslati [151]; } \\
\text { decrease: Koroneiki [12]; Gemlik, Halhalı [124]; Verdeal Transmontana [131]; } \\
\text { Memecik, Ayvalik [145] }\end{array}$ & $\begin{array}{c}\text { Increase: averaged [13]; Memecik [145]; } \\
\text { relatively constant: Picual, Hojiblanca [52]; averaged [99]; Throumbolia [117]; } \\
\text { Sarı Hasebi [124]; Chemlal, Buichret [132]; averaged [146]; Castellana [148]; } \\
\text { Ogliarola garganica [149]; Memecik [150]; Chemlali [152]; Koroneiki [153]; } \\
\text { variable: Madural [131]; Aghenfas, Mekki [132]; Picholine [139]; Chemlali [147]; } \\
\text { Oueslati [151]; } \\
\text { mostly decrease: Chemlal [132]; } \\
\text { decrease: Koroneiki [12]; Gemlik, Halhalı [123]; Koroneiki [117]; Gemlik, Halhal1 } \\
\text { [124]; Cobrançosa, Verdeal Transmontana [131]; Buža, Črna, Rosinjola [137]; } \\
\text { Memecik, Ayvalik [145]; Memecik, Edremit [150] }\end{array}$ \\
\hline
\end{tabular}


Table 3. Cont.

\begin{tabular}{|c|c|c|}
\hline \multirow[t]{2}{*}{ Sterol/Triterpene Diol } & \multicolumn{2}{|c|}{ Evolution during Ripening: Variety } \\
\hline & First Phase (RI 0-3 or Analogous) & Second Phase (RI > 3 or Analogous) \\
\hline Campestanol & $\begin{array}{c}\text { Mostly increase: Chemlal, Mekki [132]; Črna, Rosinjola [137]; } \\
\text { relatively constant: Koroneiki [12]; averaged [13]; averaged [99]; Gemlik [123]; } \\
\text { Halhalı [124]; Aghenfas, Buichret [132]; Buža [137]; averaged [146]; Chemlali [147]; } \\
\text { Ogliarola garganica [149]; Memecik, Edremit [150]; } \\
\text { variable: Halhalı [123]; Oueslati [151]; } \\
\text { decrease: Sarı Hasebi, Gemlik [124]; Memecik, Ayvalik [145] }\end{array}$ & $\begin{array}{c}\text { Increase: averaged [13]; Aghenfas [132]; Ayvalik [145]; } \\
\text { mostly increase: Buichret, Mekki [132]; Chemlali [147]; } \\
\text { relatively constant: averaged [99]; Gemlik [123]; Gemlik, Halhalı [124]; Chemlal } \\
\text { [132]; Buža [137]; Memecik [145]; averaged [146]; Ogliarola garganica [149]; } \\
\text { Memecik, Edremit [150]; } \\
\text { variable: Halhalı [123]; } \\
\text { decrease: Koroneiki [12]; Sarı Hasebi [124]; Črna, Rosinjola [137]; Oueslati [151] }\end{array}$ \\
\hline Stigmasterol & $\begin{array}{l}\text { Increase: averaged [99]; Sarı Hasebi [124]; Buža, Črna Rosinjola [137]; averaged } \\
\text { [146]; } \\
\text { relatively constant: averaged [13]; Picual, Hojiblanca [52]; Gemlik [123]; Chemlal, } \\
\text { Aghenfas [132]; Picholine [139]; Castellana [148]; Koroneiki [153]; Ogliarola } \\
\text { garganica [149]; Memecik [150]; } \\
\text { variable: Halhalı [123]; Cobrançosa, Verdeal Transmontana [131]; Mekki [132]; } \\
\text { Edremit [150]; } \\
\text { mostly decrease: Buichret [132]; Chemlali [147]; } \\
\text { decrease: Koroneiki [12]; Gemlik, Halhalı [124]; Memecik, Ayvalik [145]; Oueslati } \\
\text { [151] }\end{array}$ & $\begin{array}{c}\text { Increase: averaged [13]; Gemlik, Halhalı [123]; Gemlik [124]; Buža [137]; } \\
\text { Memecik, Ayvalik [145]; } \\
\text { mostly increase: Mekki [132]; Oueslati [151]; } \\
\text { relatively constant: Koroneiki [12]; Picual, Hojiblanca [52]; averaged [99]; } \\
\text { Aghenfas, Buichret [132]; averaged [146]; Chemlali [147]; Castellana [148]; } \\
\text { Ogliarola garganica [149]; Memecik, Edremit [150]; Koroneiki [153]; } \\
\text { variable: Cobrançosa, Madural, Verdeal Transmontana [131]; Chemlal [132]; } \\
\text { Picholine [139]; } \\
\text { decrease: Sarı Hasebi, Halhalı [124]; Črna, Rosinjola [137] }\end{array}$ \\
\hline$\Delta^{7}$-Campesterol & $\begin{array}{c}\text { Increase: Halhalı [124]; Rosinjola [137]; } \\
\text { relatively constant: averaged [99]; Sar1 Hasebi, Gemlik [124]; Buža, Črna [137]; } \\
\text { averaged [146]; Memecik, Edremit [150]; } \\
\text { variable: Gemlik, Halhalı [123]; Memecik, Ayvalik [145]; Oueslati [151]; } \\
\text { decrease: averaged [13] }\end{array}$ & $\begin{array}{c}\text { Relatively constant: averaged [13]; averaged [99]; Sarı Hasebi, Gemlik, Halhalı } \\
\text { [124]; Buža, Črna [137]; averaged [146]; Memecik, Edremit [150]; } \\
\text { variable: Gemlik, Halhalı [123]; Memecik, Ayvalik [145]; } \\
\text { mostly decrease: Oueslati [151]; } \\
\text { decrease: Rosinjola [137] }\end{array}$ \\
\hline$\Delta^{5,23}$-Stigmastadienol & $\begin{array}{l}\text { Variable: Oueslati [151]; } \\
\text { decrease: averaged [13] }\end{array}$ & $\begin{array}{l}\text { Increase: averaged [13]; } \\
\text { variable: Oueslati [151] }\end{array}$ \\
\hline Clerosterol & $\begin{array}{c}\text { Increase: averaged [13]; Throumbolia [117]; Ayvalik [145]; } \\
\text { relatively constant: averaged [99]; Halhalı [123]; Gemlik [124]; Chemlal, Aghenfas, } \\
\text { Buichret, Mekki [132]; Memecik, Ayvalik [145]; averaged [146]; Chemlali [147]; } \\
\text { Ogliarola garganica [149]; } \\
\text { variable: Gemlik [123]; Cobrançosa [131]; Edremit [150]; } \\
\text { decrease: Koroneiki [12]; Koroneiki [117]; Sarı Hasebi, Halhal [124]; Verdeal } \\
\text { Transmontana [131]; Memecik [150]; Oueslati [151] }\end{array}$ & $\begin{array}{c}\text { Increase: Sarı Hasebi, Halhalı [124]; Memecik, Ayvalik [145]; } \\
\text { relatively constant: Koroneiki [12]; averaged [13]; averaged [99]; Throumbolia, } \\
\text { Koroneiki [117]; Halhalı [123]; Gemlik [124]; Verdeal Transmontana [131]; } \\
\text { Buichret [132]; averaged [146]; Chemlali [147]; Ogliarola garganica [149]; } \\
\text { Memecik, Edremit [150]; } \\
\text { variable: Gemlik [123]; Cobrançosa, Madural [131]; Aghenfas [132]; Oueslati } \\
\text { [151]; } \\
\text { mostly decrease: Chemlal, Mekki [132] }\end{array}$ \\
\hline
\end{tabular}


Table 3. Cont

\begin{tabular}{|c|c|c|}
\hline \multirow[t]{2}{*}{ Sterol/Triterpene Diol } & \multicolumn{2}{|c|}{ Evolution during Ripening: Variety } \\
\hline & First Phase (RI 0-3 or Analogous) & Second Phase (RI > 3 or Analogous) \\
\hline$\beta$-Sitosterol & $\begin{array}{l}\text { Increase: Throumbolia [117]; Sarı Hasebi [124]; Buža, Črna, Rosinjola mg/100 g } \\
\text { [137]; Edremit [150]; Barnea, Manzanilla, Kadesh [154]; } \\
\text { relatively constant: Picual, Hojiblanca [52]; Koroneiki [117]; Memecik [145]; } \\
\text { averaged [146]; Ogliarola garganica [149]; Cornicabra [155]; } \\
\text { variable: various cultivars [14]; Gemlik, Halhalı [123]; Cobrançosa [131]; Buichret } \\
\text { [132]; Chemlali [147]; Koroneiki [153]; } \\
\text { mostly decrease: Aghenfas [132]; Picholine [139]; Chemlali [152]; } \\
\text { decrease: Koroneiki [12]; averaged [13]; averaged [99]; Gemlik, Halhalı [124]; } \\
\text { Verdeal Transmontana [131]; Chemlal, Mekki [132]; Buža, Črna, Rosinjola \% [137]; } \\
\text { Ayvalik [145]; Memecik [150]; Oueslati [151]; averaged [156] }\end{array}$ & $\begin{array}{c}\text { Increase: Koroneiki [12]; averaged [146]; Barnea [154]; } \\
\text { mostly increase: Oueslati [151]; } \\
\text { relatively constant: averaged [13]; Picual, Hojiblanca [52]; averaged [99]; Sar1 } \\
\text { Hasebi, Gemlik [124]; Chemlal, Aghenfas, Mekki [132]; Buža, Črna, Rosinjola \% } \\
\text { [137]; Memecik [145]; Ogliarola garganica [149]; Memecik, Edremit [150]; } \\
\text { Koroneiki [153]; Cornicabra [155]; averaged [156]; } \\
\text { variable: various cultivars [14]; Halhalı [123]; Cobrançosa, Madural, Verdeal } \\
\text { Transmontana [131]; Buichret [132]; Chemlali [147]; Chemlali [152]; } \\
\text { mostly decrease: Picholine [139]; } \\
\text { decrease: Throumbolia, Koroneiki [117]; Gemlik [123]; Halhalı [124]; Buža, Črna, } \\
\text { Rosinjola mg/100 g [137]; Ayvalik [145]; Manzanilla, Kadesh [154] }\end{array}$ \\
\hline Sitostanol & $\begin{array}{c}\text { Increase: Throumbolia [117]; } \\
\text { relatively constant: averaged [99]; Koroneiki [117]; averaged [146]; Memecik } \\
\text { [150]; } \\
\text { variable: Gemlik, Halhalı [123]; Chemlali [147]; Edremit [150]; } \\
\text { decrease: Koroneiki [12]; averaged [13]; Sarı Hasebi, Gemlik, Halhalı [124]; } \\
\text { Memecik [145]; Oueslati [151] }\end{array}$ & $\begin{array}{c}\text { Increase: Throumbolia [117]; Ayvalik [145]; } \\
\text { relatively constant: averaged [13]; averaged [99]; Koroneiki [117]; averaged } \\
\text { [146]; Memecik, Edremit [150]; } \\
\text { variable: Gemlik, Halhalı [123]; Chemlali [147]; Oueslati [151]; } \\
\text { decrease: Koroneiki [12]; Sarı Hasebi, Gemlik, Halhalı [124]; Memecik, Ayvalik } \\
\text { [145] }\end{array}$ \\
\hline$\Delta^{5}$-Avenasterol & $\begin{array}{c}\text { Increase: Koroneiki [12]; averaged [13]; Hojiblanca [52]; averaged [99]; } \\
\text { Throumbolia [117]; Gemlik, Halhal1 [124]; Cobrançosa [131]; Buichret, Mekki } \\
\text { [132]; Buža, Črna, Rosinjola [137]; Picholine [139]; Memecik, Ayvalik [145]; } \\
\text { Memecik, Edremit [150]; averaged [156]; } \\
\text { mostly increase: various cultivars [14]; Chemlal, Aghenfas [132]; Chemlali [147]; } \\
\text { Chemlali [152]; } \\
\text { relatively constant: Picual [52]; Gemlik [123]; averaged [146]; Ogliarola } \\
\text { garganica [149];Koroneiki [153]; } \\
\text { variable: Halhalı [123]; Verdeal Transmontana [131]; Oueslati [151]; Cornicabra } \\
\text { [155]; } \\
\text { decrease: Koroneiki [117]; Sarı Hasebi [124] }\end{array}$ & $\begin{array}{c}\text { Increase: Koroneiki [12]; Koroneiki [117]; Gemlik [123]; Sarı Hasebi, Gemlik, } \\
\text { Halhalı [124]; Rosinjola mg/100 g [137]; Buža, Rosinjola \% [137]; Ayvalik [145]; } \\
\text { Oueslati [151]; } \\
\text { mostly increase: Picual, Hojiblanca [52]; Picholine [139]; } \\
\text { relatively constant: averaged [13]; averaged [99]; Throumbolia [117]; } \\
\text { Cobrançosa [131]; Buichret [132]; Črna \% [137]; Ogliarola garganica [149]; } \\
\text { Edremit [150]; averaged [156]; } \\
\text { variable: various cultivars [14]; Madural, Verdeal Transmontana [131]; Aghenfas, } \\
\text { Mekki [132]; averaged [146]; Memecik [150]; Chemlali [152]; Cornicabra [155]; } \\
\text { mostly decrease: Chemlali [147]; } \\
\text { decrease: Halhalı [123]; Chemlal [132]; Buža, Črna mg/100 g [137]; Memecik } \\
\text { [145]; Koroneiki [153] }\end{array}$ \\
\hline
\end{tabular}


Table 3. Cont.

\begin{tabular}{cc}
\hline Sterol/Triterpene Diol & First Phase (RI 0-3 or Analogous) \\
\hline & Increase: averaged [13]; Aghenfas [132]; Edremit [150]; \\
& mostly increase: Mekki [132]; Buža, Črna, Rosinjola mg/100 g [137]; \\
& relatively constant: Koroneiki [12]; averaged [99]; Throumbolia, Koroneiki [117]; \\
Gemlik [123]; Halhalı [124]; Buža, Črna, Rosinjola \% [137]; Memecik, Ayvalik \\
[145]; averaged [146]; Ogliarola garganica [149]; Memecik [150]; \\
variable: Halhal1 [123]; Chemlal, Buichret [132]; \\
mostly decrease: Oueslati [151]; \\
$\Delta^{5,24-S t i g m a s t a d i e n o l ~}$ \\
decrease: Sar1 Hasebi, Gemlik [124]; Chemlali [147]; Chemlali [152]
\end{tabular}

Increase: Halhalı [124]; Rosinjola [137];

relatively constant: averaged [99]; Throumbolia, Koroneiki [117]; Gemlik [123];

Gemlik [124]; Aghenfas, Buichret, Mekki [132]; Črna [137]; Memecik, Ayvalik

$\Delta^{7}$-Stigmastenol

[145]; averaged [146]; Chemlali [147]; Castellana [148]; Ogliarola garganica [149] Edremit [150];

variable: Chemlal [132]; Oueslati [151];

mostly decrease: Halhalı [123];

decrease: Koroneiki [12]; Sar1 Hasebi [124]; Buža [137]; Memecik [150]

Increase: averaged [13]; Throumbolia [117]; Gemlik, Halhalı [124]; Cobrançosa

$$
\text { [131]; Ayvalik [145]; }
$$

relatively constant: Koroneiki [12]; averaged [99]; Koroneiki [117]; Chemlal,

$\Delta^{7}$-Avenasterol Mekki [132]; Memecik [145]; averaged [146]; Ogliarola garganica [149]; Edremit [150];

variable: Halhalı [123]; Memecik [150]; Oueslati [151];

mostly decrease: Aghenfas, Buichret [132]; Chemlali [147];

decrease: Gemlik [123]; Sarı Hasebi [124]; Verdeal Transmontana [131]

Increase: Sarı Hasebi, Gemlik, Halhalı [124]; Buža, Črna, Rosinjola mg/100 g [137]; Ayvalik [145];

Apparent $\beta$-sitosterol
Second Phase (RI > 3 or Analogous)

Increase: averaged [13]; Throumbolia [117]; Gemlik, Halhalı [124]; Buža \% [137];

relatively constant: averaged [99]; Koroneiki [117]; Halhalı [123]; Buichret [132]; Memecik, Ayvalik [145]; Ogliarola garganica [149]; Memecik [150];

variable: Chemlal, Aghenfas, Mekki [132]; Chemlali [147]; Oueslati [151];

Chemlali [152];

mostly decrease: Gemlik [123];

decrease: Koroneiki [12]; Sarı Hasebi [124]; Črna, Rosinjola \% [137]; averaged [146]; Edremit [150]

\section{Increase: Gemlik, Halhalı [124]}

relatively constant: Koroneiki [12]; averaged [99]; Throumbolia, Koroneiki [117]

Sarı Hasebi [124]; Chemlal, Buichret, Mekki [132]; Buža, Črna [137]; Memecik,

Ayvalik [145]; averaged [146]; Chemlali [147]; Castellana [148]; Ogliarola

garganica [149]; Memecik, Edremit [150]; variable: Gemlik [123]

mostly decrease: Aghenfas [132]; Oueslati [151]; decrease: Halhalı [123]; Rosinjola [137]

Increase: Gemlik, Halhalı [124]; Ayvalik [145];

relatively constant: averaged [13]; averaged [99]; Koroneiki [117]; Chemlal,

Aghenfas, Buichret [132]; Memecik [145]; averaged [146]; Chemlali [147];

Ogliarola garganica [149]; Edremit [150];

variable: Gemlik, Halhalı [123]; Verdeal Transmontana [131]; Mekki [132];

Memecik [150]; Oueslati [151];

mostly decrease: Madural [131]

decrease: Koroneiki [12]; Throumbolia [117]; Sarı Hasebi [124]

Increase: Sarı Hasebi [124];

relatively constant: Gemlik, Halhalı [123]; Castellana [148]; Ogliarola garganica [149];

decrease: Gemlik, Halhalı [124]; Buža, Črna, Rosinjola mg/100 g [137]; Rosinjola $\%$ [137]; Memecik, Ayvalik [145]; averaged [146]
[146]; Castellana [148]; Ogliarola garganica [149]; variable: Halhalı [123] 
Table 3. Cont.

\begin{tabular}{|c|c|c|}
\hline \multirow[t]{2}{*}{ Sterol/Triterpene Diol } & \multicolumn{2}{|c|}{ Evolution during Ripening: Variety } \\
\hline & First Phase (RI 0-3 or Analogous) & Second Phase (RI $>3$ or Analogous) \\
\hline Total sterols [mg/kg] & $\begin{array}{l}\text { Increase: averaged [13]; Sarı Hasebi, Gemlik, Halhalı [124]; Buža, Črna, Rosinjola } \\
\text { [137]; Edremit [150]; averaged [156]; } \\
\text { relatively constant: Hojiblanca [52]; averaged [99]; Halhalı [123]; Chemlal, } \\
\text { Aghenfas, Mekki [132]; Memecik, Ayvalik [145]; averaged [146]; Castellana [148]; } \\
\text { Koroneiki [153]; } \\
\text { variable: Gemlik [123]; Cobrançosa [131]; Buichret [132]; Chemlali [147]; } \\
\text { Chemlali [152]; } \\
\text { mostly decrease: Oueslati [151]; } \\
\text { decrease: various cultivars [14]; Picual [52]; Verdeal Transmontana [131]; } \\
\text { Ogliarola garganica [149]; Memecik [150] }\end{array}$ & $\begin{array}{l}\text { Increase: Sarı Hasebi, Gemlik, Halhalı [124]; Oueslati [151]; averaged [156]; } \\
\text { relatively constant: Hojiblanca [52]; averaged [99]; Chemlal, Aghenfas, Mekki } \\
\text { [132]; Ayvalik [145]; averaged [146]; Castellana [148]; Memecik [150]; Chemlali } \\
\text { [152]; Koroneiki [153]; } \\
\text { variable: various cultivars [14]; Gemlik, Halhalı [123]; Cobrançosa, Madural [131]; } \\
\text { Buichret [132]; Edremit [150]; } \\
\text { mostly decrease: Verdeal Transmontana [131]; Chemlali [147]; } \\
\text { decrease: averaged [13]; Picual [52]; Buža, Črna, Rosinjola [137]; Memecik [145]; } \\
\text { Ogliarola garganica [149] }\end{array}$ \\
\hline Erythrodiol & $\begin{array}{l}\text { Increase: Buža, Rosinjola [137]; } \\
\text { relatively constant: Koroneiki [12]; Črna [137]; averaged [146]; } \\
\text { mostly decrease: Oueslati [151] }\end{array}$ & $\begin{array}{c}\text { Increase: Koroneiki [12]; Buža, Črna, Rosinjola [137]; } \\
\text { mostly decrease: Oueslati [151]; } \\
\text { decrease: averaged [146] }\end{array}$ \\
\hline Uvaol & $\begin{array}{c}\text { Relatively constant: averaged [146]; } \\
\text { variable: Buža, Črna, Rosinjola [137]; Oueslati [151]; } \\
\text { decrease: Koroneiki [12] }\end{array}$ & 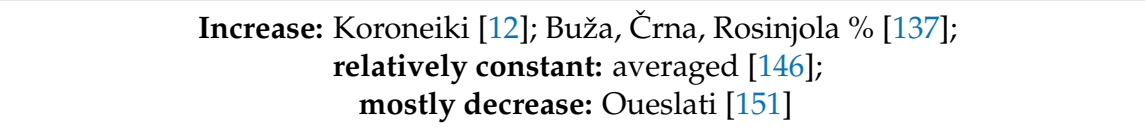 \\
\hline Erythrodiol+uvaol & $\begin{array}{c}\text { Increase: Mekki [132]; } \\
\text { mostly increase: Buichret [132]; Ogliarola garganica [149]; } \\
\text { relatively constant: averaged [99]; Ayvalik [145]; averaged [146]; } \\
\text { variable: Gemlik, Halhalı [123]; } \\
\text { mostly decrease: Chemlal [132]; } \\
\text { decrease: averaged [13]; Sar1 Hasebi, Gemlik, Halhalı [124]; Aghenfas [132]; } \\
\text { Memecik [145]; Memecik, Edremit [150] }\end{array}$ & $\begin{array}{l}\text { Increase: Sarı Hasebi, Gemlik, Halhalı [124]; Memecik, Ayvalik [145]; } \\
\text { relatively constant: averaged [99]; Chemlal [132]; Ogliarola garganica [149]; } \\
\text { Memecik, Edremit [150]; } \\
\text { variable: Gemlik, Halhalı [123]; } \\
\text { mostly decrease: Buichret [132]; } \\
\text { decrease: averaged [13]; Aghenfas, Mekki [132]; averaged [146] }\end{array}$ \\
\hline
\end{tabular}

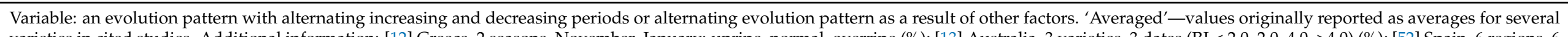

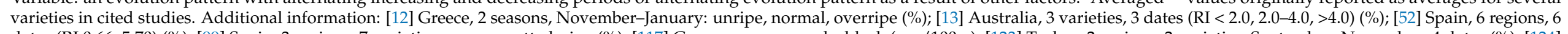

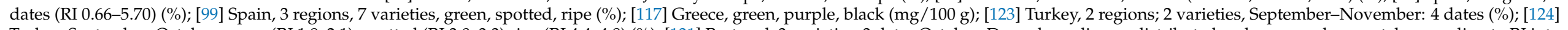

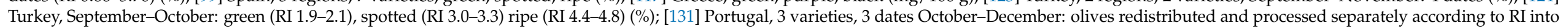

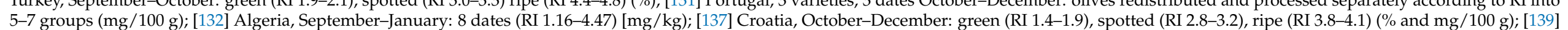

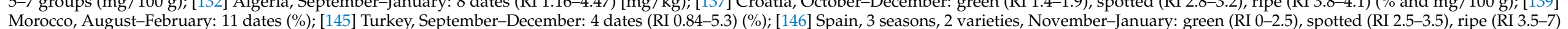

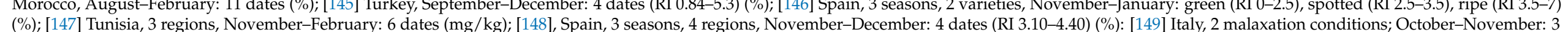

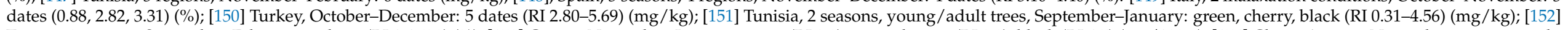

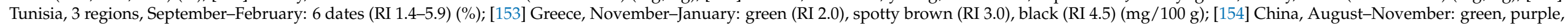
and black (RI 2.1-5.3) [mg/kg]; [155], Spain, 4 seasons, 3-5 dates (RI 0.6-5.3) (\%); [156] Spain, 4 varieties, analyzed directly in olive fruit flesh and not oil, September-December 4 dates (RI 0.3-3.9) (\%). 
It is of interest for olive growers and oil producers to conduct harvest when both oil quantity and quality are closest to their peak. In practice, harvest time is traditionally determined based on the visible fruit color, which is not always reliable [143]. The olive ripening index (RI), also known as the maturation index (MI), is a more reliable measure for estimating the ripening/maturity degree of the fruits. It is determined by evaluating the color of the skin and pulp of 100 olives randomly chosen as representative of a larger quantity [157]. However, it was demonstrated many times that the development and ripening of olive fruit and the contents of various chemical compounds that are being biosynthesized do not always correlate, significantly depending on variety, growing season, and region, as well as other factors $[143,158]$. For these reasons, research on the changes in yield and chemical composition of oil during olive ripening, including the content and composition of sterols and triterpene diols, is an ever-growing field of scientific interest of great practical relevance.

A significant effect of the olive ripening degree on the content and composition of sterols and triterpene diols in olive oil was observed in many studies (Table 3). As the majority of the published studies included three ripening degrees, with the olives used for production in most cases denominated as 'green', 'spotted', and 'ripe', respectively, or similar, the description of their evolution is divided into two parts, i.e., periods before and after color change (with the boundary set at 'spotted' stage or equivalent). In cases where the investigated oils were obtained from olives harvested at more than three ripening degrees, the studied period was divided into two parts on the basis of the reported ripening indices (when available; boundary set at RI around 3) or, in a fewer number of cases, into two periods of equal duration. The dynamics of the accumulation of sterols and triterpene diols in each of the two stages are described in Table 3 by the following phrases: 'increase', 'mostly increase', 'relatively constant', 'variable', 'mostly decrease', and 'decrease'. The information about the varieties in question and some additional data from the reviewed studies are also reported.

It should be noted that the reported studies differed with respect to the country and region in which they were conducted, meaning each was characterized by its own agroecological conditions predetermined by geographical origin and location. Furthermore, the variability was amplified by a wide time range in which the studies were performed (few decades), with many growing seasons, each characterized by unrepeatable weather conditions. Consequently, the time frames encompassing the studied ripening periods were different, ranging from longer, i.e., from August to February with 11 harvest dates [139], to shorter ones, including three dates in October and November [124]. It must be also kept in mind that certain studies reported the changes in sterol relative proportions, while other reported concentrations. From these aspects, it is necessary to take caution when comparing published studies of such diverse backgrounds.

A number of studies included several varieties monitored in the same or at least very similar ripening conditions. In many such cases, the evolution patterns of sterols and triterpene diols during ripening significantly differed among varieties (Table 3). Particular interfering effects of other factors were also observed. For example, in a four season study, the total sterols' concentration in Cornicabra olive oils slightly decreased during ripening in two seasons; a more considerable decrease was observed in a single one, while in another, a major increase was recorded [155].

A great variability in the dynamics of accumulation was observed for the majority of sterols and triterpene diols, while for some, a tendency toward particular repeating patterns was noted despite the abovementioned probable interferences. The level of cholesterol was found to be mostly constant throughout the whole period of ripening in the majority of studies (Table 3). The behavior of brassicasterol, one of the least abundant sterols in olive and olive oil, was monitored in a relatively small number of studies. It was found to be mostly constant, although a number of studies observed an increase in the later stages of ripening. It is possible that the activity of a particular C22-desaturase was more expressed in the later stages by catalyzing the conversion of campesterol into 
brassicasterol (Figure 2) [15]. Olive oils of particular varieties that exhibited an increase in brassicasterol levels in the later stages of ripening, such as Turkish Gemlik, Halhali [124], and Memecik [150], were also characterized by a decrease in campesterol levels in the same period. Although the accumulation of 24-methylenecholesterol in different studies was rather diverse, the majority reported a rather constant level, while a decreasing pattern was observed in the lowest number of cases. The level of one of the major sterols, campesterol, was constant or variable throughout ripening in the majority of studies and it was only rarely found to increase. Its decrease was more often found in the second half of the ripening cycle. The behavior of campestanol was similar, with more studies observing an increase in the second than in the first phase of ripening. Levels of $\Delta^{7}$-campesterol were rather constant or variable throughout ripening. Similarly to the majority of other sterols and triterpene diols, the levels of stigmasterol did not change significantly in most cases, but in several studies, an increase was observed, especially in the later stages of ripening. In some cases, a decrease in stigmasterol was observed in the initial phase. Clerosterol levels were mostly constant, although with a larger number of studies observing a decrease in its level in the first part of the ripening cycle. The variability in the accumulation of $\beta$-sitosterol was also high, but, contrary to the majority of sterols and triterpene diols, a relatively large number of studies observed a decrease in the first phase, while several studies reported such a behavior in the second phase as well. Interestingly, in the majority of cases, $\beta$-sitosterol did not follow the same pattern in both ripening phases, suggesting there was a shift in its biosynthetic flux at a certain point, possibly affected by other factors, such as oil accumulation in the fruit, its participation in other plant biosynthetic pathways, and so on. The behavior of sitostanol was in most cases characterized by a steady level or a decrease, while an increase was noted rarely. As suggested by Sakouhi et al. [159], the decrease in stanols may be due to their conversion into steroidal hormones, such as brassinosteroids, which regulate plant growth and development [19]. The first part of the ripening cycle was in many studies marked by an increase in $\Delta^{5}$-avenasterol level, while a decrease was rarely noted. In several studies, an increase in $\Delta^{5}$-avenasterol level was observed in the second phase as well. As in the case of $\beta$-sitosterol and some other sterols, in several cases, a shift towards patterns different than those observed in a previous phase was recorded. $\Delta^{5,24}$-Stigmastadienol, $\Delta^{7}$-stigmastenol, and $\Delta^{7}$-avenasterol exhibited a large variety of behaviors. A noted decrease in $\Delta^{7}$-sterol $/ \Delta^{5}$-sterol ratio was previously explained by the moderation of biosynthesis by the plant according to its needs in regulating growth and development [159]. In most studies, the level of apparent $\beta$-sitosterol was constant in the first part of the ripening period, an increase was observed in several studies, while no study reported a decrease. After a change in color or equivalent, a decrease in its level was observed in particular cases, while an increase was recorded only once. Contrasting results were published for erythrodiol and uvaol. For particular varieties, an increase in erythrodiol and uvaol levels was observed in oils obtained from ripe olives, which was explained by the fact that these triterpene diols are mostly located in olive skin, which becomes more fragile as the fruit ripens and fragments better during milling [99].

Similarly to the behavior of the majority of sterols, the content of total sterols changed in many ways. Again, the dynamics of the accumulation of the total sterols content rarely maintained the same course during the whole ripening period, although some exceptions were noted. It was observed in several studies that many sterols, as well as the total sterol concentration, peak at a certain point during ripening, after which they decrease. Sakouhi et al. [159] stated that, in the later phases of ripening, the activity of enzymes involved in the biosynthesis of sterols decreases, while the conversion of sterols to stanols and steryl esters in the same period is increased. Simultaneously, oil is accumulated during most parts of the ripening period and the compounds from the unsaponifiable fraction are practically being 'diluted', which may contribute to a decrease in the concentration of sterols and triterpene diols [99]. Gutierrez et al. [52] observed a constant decrease in the concentration of total sterols. Such a pattern was more pronounced for Picual than for Hojiblanca variety, proving once again that variety is an important factor. It is 
probable that the enzymatic pools and loads, as well as their evolution during ripening, which are specific for a particular variety, most directly predetermine the dynamics of biosynthesis and final quantities of sterols and triterpene diols in different monovarietal olive oils. Further, different varieties were previously shown to exhibit different dynamics of accumulation of oil during ripening. Ranalli et al. [160] observed the percentage of oil in olive drupes peaking at RI of around 5 (black skin and violet flesh) for Leccino and at RI of almost 4 (mostly black-reddish skin and green pulp) for Frantoio variety, suggesting not only enzymatic activity, but also the factor of 'dilution' of sterols by saponifiables over time is genetically predetermined. The oscillations in total sterol concentrations during ripening were also attributed to their conversion into steroidal hormones and vitamins [159]. In some cases, the content of total sterols was found to increase in the later stages of ripening. The results from Taticchi et al. [149] suggested that the extractability of sterols from olive cells into the water/oily phase at late ripening stages might be facilitated by high levels of depolymerizing enzymatic activities allowing a better disruption of different fruit tissues during milling.

In contrast to the majority of studies in which conclusions were drawn based on the content and/or composition of sterols and triterpene diols in the extracted oils, FernándezCuesta et al. [156] monitored the evolution of squalene and sterols during olive ripening directly in olive flesh. They noted an increase in total sterol amounts during the whole period from September to December across four ripening degrees, with RI varying between 0.3 and 3.9. Interestingly, an increase in squalene concentration lasted throughout the whole experiment as well, although it was less intense with a lower increase in percentage compared with that of sterols in all the ripening phases except the last. Squalene is a precursor to all sterols and triterpene diols, so it is reasonable to assume that the increase in its concentration throughout ripening was slower because its fraction was being converted to triterpenoids, including sterols. The proportion of $\beta$-sitosterol decreased and that of $\Delta^{5}$-avenasterol increased simultaneously with the increase in the proportion of oil in the fruit, while in the last stage, when the accumulation of oil apparently stopped and the fresh fruit weight became constant or even slightly decreased owing to the evaporation of water, the $\beta$-sitosterol $/ \Delta^{5}$-avenasterol ratio evened off. The study included four varieties and some inter-varietal differences in the evolution patterns were observed, but mostly slight, allowing the previously mentioned general conclusions. On the other hand, quantitative differences between varieties were significant. Fernández-Cuesta et al. [156] pointed out that the squalene amount was already high at the beginning of the ripening period covered in the study, and concluded that squalene accumulation in olives is scarcely related to this period of fruit maturation. Such a result was in contrast with the findings from Sakouhi et al. [161], who reported maximum squalene level at 21 weeks after flowering, which was followed by a dramatic decrease measured over the five following weeks, finally reaching a concentration ten times lower than the maximum in the 38th week after flowering. The authors explained the decrease in squalene concentration as a consequence of its consumption in the biosynthesis of sterols and other triterpenoids, which was corroborated by a significant increase in total sterols noted in the same period (21st and 26th week after flowering), after which a rather sharp decrease in their concentration was observed [161]. It must be noted that the two mentioned studies differed with respect to the matrix from which squalene was extracted (lyophilized fruits vs. soxhlet extracted oil) and other details, which could have caused the mentioned differences. Furthermore, other factors, such as variety, region, season, and so on, could have contributed. Orozco-Solano et al. [162] analyzed fruits from three olive varieties in a period from September to January across five ripening degrees (green, green-yellow, yellow-purple, purple, and black) and observed different behaviour of the majority of sterols depending on the variety.

A strong negative correlation between $\beta$-sitosterol and $\Delta^{5}$-avenasterol was noted in the majority of the reported studies (Table 3). Aparicio and Luna [140] explained such a phenomenon based on the decrease in stone/flesh ratio in the fruits during ripening, resulting in a lower proportion of oil from stone, which has a much higher $\beta$-sitosterol $/ \Delta^{5}$ - 
avenasterol ratio than that from flesh [111]. Another possibility is that the activity of particular enzymes wanes in a later stage [159], which possibly includes $\Delta^{24}$-isomerase/reductase, which converts $\Delta^{5}$-avenasterol to $\beta$-sitosterol [15]. Gutiérrez et al. [52] suggested the possible activity of a desaturase that transforms $\beta$-sitosterol into $\Delta^{5}$-avenasterol.

Many studies confirmed that various varieties exhibit different dynamics of sterol accumulation during ripening (Table 3). However, a few of them showed, at least when the effects of other factors were prevented or minimized, a great similarity in the relative changes in ripening patterns despite quantitative differences in the sterol content. Fuentes de Mendoza et al. [146] showed that it is possible to differentiate olive oils according to ripening degree (green, spotted, ripe) using LDA with particular fatty acids, triglycerides, and sterols as variables, despite samples belonging to two different varieties. Among compounds from other chemical families, $\beta$-sitosterol and $\Delta^{5}$-avenasterol were extracted by a LDA model as those with a significant discriminating power. Orozco-Solano et al. [162] observed a certain degree of grouping of olive oil samples from three varieties according to their ripening degree by applying PCA on sterol and aliphatic alcohol compositional data. In another study, a relatively successful discrimination of oils according to ripening degree was achieved by applying both PCA and DA, although a significant interaction with the age of olive tree as the other factor was noted, which resulted in the formation of sub-classes visible on the PCA and DA plots. Besides sterols and triterpene diols, quantitative data for other compounds, such as squalene, chlorophylls, carotenoids, and aliphatic alcohols, were used as variables in performing chemometric analysis [151].

Koutsaftakis et al. [12] succeeded in grouping olive oils of Koroneiki variety according to ripening degree using CDA with solely sterol compositional data, despite the samples belonging to two consecutive growing seasons. Another study succeeded in obtaining good PCA separation of oils from three different varieties made of olives harvested at three ripening degrees using only sterols and triterpene diols as variables [137]. To further minimize the effect of quantitative differences, the authors introduced the ratios of concentrations and/or percentages of particular sterols and diols as variables. PCA, with sitostanol/uvaol, uvaol/stigmasterol, $\Delta^{7}$-campesterol $/ \beta$-sitosterol, and clerosterol $/ \Delta^{5}$-avenasterol as variables, allowed the best separation of fresh oils according to ripening degree. The first three were selected by LSD as those with the highest differentiating power. When oils stored for a year were introduced into the dataset along with the fresh ones, the information about ripening degree was retained mostly in the levels of 24 -methylenecholesterol/ $\beta$-sitosterol, erythrodiol, campestanol/stigmasterol, $\Delta^{5}$-avenasterol, stigmasterol $/ \Delta^{7}$-campesterol, and 24-methylenecholesterol [137].

\subsection{Influence of Other Factors}

Variety and ripening degree are certainly among the most, if not the most, important factors influencing the content and composition of sterols and triterpene diols in olive oil. However, harvest year and geographical origin, inseparably related to the given pedoclimatic conditions, as well as agronomic and technological parameters, were shown to have an important influence as well. As this review article focuses mostly on the influence of variety and ripening degree, this part is not equally comprehensive, but instead reports particular examples to gain a better insight into the diversity of factors affecting the final olive oil chemical composition and quality, particularly the content and composition of sterols and triterpene diols.

Many published studies reported the effect of the growing region on the content and composition of sterols and triterpene diols. The levels of particular major sterols, such as $\beta$-sitosterol, campesterol, and stigmasterol, as well as total sterols, were found to decrease at higher altitudes, while for particular triterpenic alcohols, including the sterol precursor cycloartenol, the opposite was found, implying a slowdown in sterol biosynthesis in such conditions [163]. It was shown that total sterols negatively correlate with limestone percentage in soil [164]. A higher level of $\Delta^{7}$-stigmastenol was found in oils produced from olives grown on red clay soil in comparison with white lime [55]. Despite the fact that, in 
lots of studies that investigated, the influence of geographical origin on the effect of other factors was not eliminated, there is a consensus that a significant variability is related to the different climate conditions at each growing area, such as rainfall, temperature, and humidity, as well as to soil properties, particularly $\mathrm{pH}$, limestone content, and deficiency in certain elements, among others [2].

Lazzez et al. [152] compared olive oils from Chemlali variety during four harvest seasons produced at six ripening degrees in three growing regions with different pedoclimatic conditions. They found the growing region to account for more variability than ripening degree and the two factors significantly interacted. Growing season had no significant effect. In other studies, harvest year had a significant effect on the majority of sterols and triterpene diols [13], although significantly interacting with other factors $[102,104,105,115,121,151,165]$. In a study with seven Spanish varieties grown in five growing regions, in a direct comparison variety had a stronger influence than geographical location on $\beta$-sitosterol, $\Delta^{5}$-avenasterol, and total sterols, expressed as a percentage of variance [74].

Like in the case of variety, many studies more or less successfully aimed to find chemical markers of geographical origin among sterols and triterpene diols using univariate and multivariate statistical techniques. Significant differences in sterol and triterpene diol composition and total sterol content were found between oils obtained from olives from 10 locations with different pedoclimatic characteristics in north Tunisia by ANOVA and a post-hoc test [166]. Two olive varieties grown in two regions in Turkey harvested at four different ripening degrees were studied for their sterol content and composition. Campesterol, stigmasterol, $\Delta^{5}$-avenasterol, $\beta$-sitosterol, $\Delta^{5,24}$-stigmastadienol, erythrodiol + uvaol, and total sterols contents were significantly affected by all the three factors, with minor exceptions [123].

In a study with Australian olive oils produced from olives from four regions selected to represent a range of extremes of conditions across olive growing areas within Australia, the growing region was found to have a significant effect on the composition, i.e., proportions of particular sterols. Variety also had a significant effect, while the influence of growing season and ripening degree was much weaker. The factors variety and growing region interacted, as the effect of particular region was not the same for all the varieties, and vice versa. Generally, the proportion of stigmasterol was higher in the northern growing regions [134]. Together with FAMEs, $\beta$-sitosterol, $\Delta^{5}$-avenasterol, and stigmasterol contributed to the differentiation of olive oils from various locations in Sicily, Italy by chemometric techniques of PCA and SIMCA classification [167].

Monovarietal olive oils from four Greek islands with a similar climate were compared. Although significant differences were found for $\beta$-sitosterol, sitostanol, $\Delta^{5}$-avenasterol, and total sterols, ANOVA and post-hoc tests found no variable able to distinguish all four regions. PCA showed a modest grouping of oils according to growing region with partial overlapping, but a good differentiation was obtained by discriminant function analysis (DFA) with $\beta$-sitosterol and sitostanol included in the model with compounds from other chemical families. The models that used FAMEs and triacylglycerols were more successful than the one that used sterols as the only variable [168]. The same group of authors found sterols to be more important and successful in differentiating Apulian olive oil (Italy) from three growing locations [169]. The authors applied several chemometric techniques and each of them extracted a number of sterols as important variables for geographical discrimination, including campestanol, $\Delta^{7}$-stigmastenol, sitostanol, $\beta$-sitosterol, campesterol, $\Delta^{7}$-avenasterol, clerosterol, $\Delta^{5}$-avenasterol, and 24-methylene-cholesterol.

When olive oils obtained from three varieties grown in two growing areas in Iran were compared by ANOVA and PCA, significant differences were found, with variety and growing region interacting for the majority of sterols, except for $\beta$-sitosterol having a higher and $\Delta^{5}$-avenasterol having a lower proportion in oils from all three varieties grown in Qom in relation to those grown in Gorgan region [136]. 
Bajoub et al. [170] aimed to attain a geographical discrimination of virgin olive oils from Picholine Marocaine variety produced in seven north Moroccan regions in two growing seasons based on their physico-chemical quality and compositional parameters, including sterols and triterpene diols, using a multivariate data analysis approach. Significant differences according to growing region were determined for all the sterols and total sterols, except brassicasterol and sitostanol. Among other compounds, CDA extracted $\Delta^{5,23}$-stigmastadienol, campestanol, $\Delta^{7}$-stigmastenol, $\Delta^{5,24}$-stigmastadienol, $\beta$-sitosterol, $\Delta^{5}$-avenasterol, $\Delta^{7}$-campesterol, cholesterol, erythrodiol, uvaol, and $\Delta^{7}$-avenasterol among the most discriminating variables, while some other sterols contributed less.

Particular studies investigated the differences between olive oils originating from different countries. In a study of Giacalone et al. [171], extra virgin olive oils of Italian and non-Italian origin (from Spain, Tunisia, and blends of EU origin) were differentiated by the contents and composition of free and esterified sterols by chemometric tools. Esterified sterols turned out to be more useful differentiators, with campesteryl-C18 found to be highly significant for defining non-Italian samples. Tunisian and Italian olive oils were confronted using HCA and OPLS-DA chemometric techniques after analysis of sterols by untargeted metabolomics analysis using ultra-high-pressure liquid chromatography with electrospray quadrupole-time-of-flight hybrid mass spectrometry (UHPLC-ESI/QTOFMS), and many unknown sterols were found to be good differentiators according to country of origin [87]. A similar metabolomics-based approach was applied in another study where virgin olive oils from six Italian growing regions were differentiated [86]. An enormous number of sterols were tentatively identified and the authors pointed out a possibility that some of them originated from the oil microbiota, typically present in solid particles and micro drops of vegetation water, where yeasts are predominant. OPLS-DA extracted many potential differentiators based on geographical region, among which iokundjoside (from the sterol sub-class of cardanolides) and solaspigenin (a spirostanol) were found to have the highest discriminating power. It is worth mentioning that, in this study, only $13.6 \%$ of sterols were useful for both varietal and geographical differentiation, suggesting mostly different mechanisms were affected by the two factors.

Agronomic factors in olive orchard management other than ripening degree and time of harvest also significantly affect the quality of olive fruits and, therefore, the final quality of fresh olive oil [2].

A significant effect of organic versus non-organic cultivation was determined for a number of sterols, although significantly depending on growing season and ripening degree [165]. Chtourou et al. [151] compared olive oils obtained from olives from 'young' (five years old) and 'adult' (50 years old) trees planted at the same pedoclimatic conditions and harvested at three ripening degrees, as 'green', 'cherry', and 'black'. The effects of all three factors were significant for the majority of sterols. Age of trees significantly influenced the concentration of cholesterol, campesterol, clerosterol, $\beta$-sitosterol, sitostanol, $\Delta^{5,24}$-stigmastadienol, and total sterols, as well as their precursor, squalene. The total sterol concentration was higher in oils from adult trees. The DA model successfully differentiated olive oils based on ripening degree, with sub-clusters formed according to the age of trees for each RI visible on a two-dimensional DA plot. Age of trees significantly affected the accumulation of sterols during ripening, and oils of some age/RI combinations were characterized by the proportions of particular sterols outside the regulatory limits. The authors pointed out the importance of evaluating and taking into account the age of olive trees together with other factors when estimating optimal harvest dates.

Water stress and irrigation were shown to have a significant influence on sterols and triterpene diols in olive and olive oil. Complementary irrigation $(80 \mathrm{~mm})$ applied only during the third stage of olive ripening delayed ripening time and increased fruit size and oil content. Prolonged water deficit reduced fruit growth, oil accumulation rate, and the content of potassium in the fruit, and enhanced ripening and pre-harvest fruit drop. As a side-effect, oils obtained from olives from irrigated trees contained decreased concentrations of campesterol, campestanol, $\beta$-sitosterol, and $\Delta^{5}$-avenasterol [172]. In another 
study, oils produced from water stressed olive fruits contained higher proportions of campesterol, campestanol stigmasterol, $\beta$-sitosterol, and sitostanol, and lower proportions of $\Delta^{5}$-avenasterol, $\Delta^{7}$-avenasterol, and $\Delta^{7}$-stigmastenol than those obtained from olives from irrigated trees. Water-stressed fruits yielded oils with a higher total sterol concentration [173]. Similar results were obtained by Stefanoudaki et al. [174], where irrigation generally caused a significant, but relatively small increase in the proportions of the majority of sterols, except $\Delta^{5}$-avenasterol and $\Delta^{5,24}$-stigmastadienol, simultaneously causing a large decrease in the content of total sterols from 1604 to $1005 \mathrm{~g} / \mathrm{kg}$. Opposite to those studies, Guillaume et al. [13] observed changes in the proportions of particular sterols caused by irrigation, but total sterols were not affected.

The influence of crop load, ranging from a full yield of $26.2 \mathrm{~kg}$ per tree to a reduced yield of $12.5 \mathrm{~kg}$ per tree, was found to be insignificant with respect to the amount of sterols, despite that fruits of trees with the lowest yield reached full maturity at the so-called 'black' ripening degree 30 days earlier and accumulated more oil faster [175]. The levels of $\beta$-sitosterol, sitostanol, and triterpene diols were significantly lower, and those of $\Delta^{5}$ avenasterol and $\Delta^{7}$-avenasterol were significantly higher in olive oils obtained from fruits of larger size versus fruits of smaller size [13].

Particular sterols and especially triterpene diols were found to be significantly affected by the delay between olive harvest and processing. Erythrodiol + uvaol and stigmasterol levels increased and the campesterol level decreased after a longer delay period [13]. A similar increase was observed for $\Delta^{7}$-stigmastenol [55]. By comparing the composition of oil in different fruit tissues, Guillaume et al. [13] found that the contents of $\Delta^{7}$-stigmastenol and erythrodiol+uvaol were higher in the skin/outer flesh fraction, which, according to the authors, can explain why the levels of those sterols tend to increase in oils produced in nonoptimum processing conditions that include longer malaxing times, higher temperatures, or time delays between harvesting and crushing.

Olive paste malaxation parameters were shown to have an impact, but generally of a lower degree than that caused by variety, ripening degree, and environmental and agronomic factors. In a study by Guillaume et al. [13], erythrodiol + uvaol, stigmasterol, and $\Delta^{7}$-stigmastenol were the only compounds affected, with increased concentrations found in oil after a longer malaxation duration (60 min vs. 30 and $15 \mathrm{~min}$ ). A higher malaxing temperature $\left(38^{\circ} \mathrm{C}\right.$ vs. $28^{\circ} \mathrm{C}$ and $\left.18^{\circ} \mathrm{C}\right)$ caused an increase in erythrodiol + uvaol, stigmasterol, and $\Delta^{7}$-campesterol proportions as well as total sterol amount. In another study, higher malaxation temperature $\left(45^{\circ} \mathrm{C}\right.$ vs. $\left.30^{\circ} \mathrm{C}\right)$ also extracted more stigmasterol [12]. Oil obtained by malaxation at $30^{\circ} \mathrm{C}$ could have been considered as of better quality, as it was characterized by a higher campesterol/stigmasterol ratio, as stigmasterol content is considered to be higher in defective oils. These oils were also significantly richer in $\Delta^{5}$ avenasterol. Di Giovacchino et al. [176] did not find any effects of malaxation duration and temperature on the composition of sterols. Higher pressing temperatures in a traditional olive processing system resulted in an increased $\Delta^{7}$-stigmastenol proportion [55].

Ranalli et al. [177] compared the quality and composition of different oil fractions obtained during olive processing, that is, oil from percolation (first fraction) and oil from centrifugation (second fraction). The first fraction olive oil was characterized by a higher ratio of campesterol/stigmasterol and lower concentration of total sterols and triterpene alcohols. In a more recent study, different extraction systems were compared (pressing, centrifugation with and without mill waste water recycling) in processing olives from various ripening degrees [178]. A relatively weak effect of extraction technology on sterol and triterpene diol composition, especially on $\beta$-sitosterol and $\Delta^{5}$-avenasterol, was observed in relation to that induced by ripening degree. The effects of the two factors interacted. Koutsaftakis et al. [12] found that oils obtained by two- or three-phase centrifugation systems contained a higher campesterol proportion, while those obtained by a traditional pressing system were characterized by higher levels of $\beta$-sitosterol and stigmasterol. It should be pointed out that the observed differences were minor. The effectiveness of an innovative ultrasound-assisted extraction system was tested at industrial scale [149]. Olives obtained 
at different ripening degrees were processed and the ultrasound system extracted more total sterols than a standard one only at the last ripening stage, probably as a consequence of softer olive tissues due to an increased level of depolymerizing enzymatic activities, as suggested by the authors. The sterol composition was not affected.

The influence of storage on the content and composition of sterols and triterpene diols was not studied extensively. Thanh et al. [179] found $\beta$-sitosterol level in olive oil to decrease by $16.5 \%$ after 6 months of storage at room temperature. The levels of campesterol, $\beta$-sitosterol, and stigmasterol were quite stable after several weeks at $55^{\circ} \mathrm{C}$, did not change after $1 \mathrm{~h}$ at $100{ }^{\circ} \mathrm{C}$, but had decreased by around $60 \%$ after $1 \mathrm{~h}$ at $200^{\circ} \mathrm{C}$. Abu-Alruz et al. [55] found an increase in $\Delta^{7}$-stigmastenol level after 6 months of storage in different containers. In another study, after a 12-month storage of virgin olive oils at different temperatures (room, $+4{ }^{\circ} \mathrm{C},-20^{\circ} \mathrm{C}$ ) a decrease in stigmasterol, campesterol, $\Delta^{5,24}$-stigmastadienol, uvaol, and apparent $\beta$-sitosterol, as well as an increase in $\Delta^{7}$-stigmastenol and campestanol levels, was noted [137].

\section{Conclusions}

The content and composition of sterols and triterpene diols is of great importance for various aspects of virgin olive oil quality. These compounds are among the parameters tested to verify the authenticity of olive oil and detect counterfeiting to meet the requirements of the regulation and trade standards. Sterols are considered among the most valuable ingredients of olive oil owing to their bioactivity and multiple beneficial effects on human health. Furthermore, sterols and triterpene diols have shown a significant potential as chemical markers useful for ensuring traceability and guaranteeing the varietal and geographical origin of olive oil.

Many studies have shown that the varietal diversity of olives is extremely rich, with genetically predetermined enzymatic load and activity, producing oils with different profiles of sterols and triterpene diols. Although a large number of studies on this topic have been published in general, when divided by the number of varieties included, it becomes evident that the number of studies per variety is actually small and that most varieties are still unexplored. In addition, the proven interaction between variety and other factors, such as ripening degree, geographical origin, growing season, agronomic and technological parameters, and so on, that also significantly affect the composition of olive oil make quite difficult the achievement of robust, reliable, and consistent varietal characterization and differentiation.

Olive ripening degree plays a decisive role in determining the final composition and quality of olive oil. Certain repetitive patterns during ripening have been observed for particular sterols and triterpene diols, but the data are very diverse, as this largely depends on variety and other factors. A lot of work on this topic has been published overall; however, it can again be said that, for any given variety, a small number of studies have been conducted that investigated the influence of ripening. Therefore, at this point, more general and definitive conclusions were not possible.

Obviously, if more practical conclusions are to be sought, a more systematic approach is needed, with experiments repeated in many harvest years and involving all the major interfering factors. This would enable a more precise identification of varieties that at certain ripening degrees yield oils with a high sterol content as an added nutritional value. Varieties with interesting specific properties could be selected and used in olive growing programs for propagation and the creation of new ones with enhanced characteristics. On the other hand, such improved knowledge would enable better management of varieties that are, in certain conditions, prone to yield oils in which the natural composition of sterols and/or triterpene diols does not meet the legally prescribed limits. In this way, potential problems in their trade could be anticipated and avoided.

Another important direction in future research on sterols and triterpene diols could certainly be the identification of novel compounds from this group, in free and bound form, which would provide more information and enable developing more precise chemometric 
differentiation models applicable for ensuring virgin olive oil traceability and authenticity. The use of the metabolomics approach with powerful sophisticated analytical techniques and big data processing tools is expected to become more common in the near future in delivering such results.

Author Contributions: Conceptualization, M.L. and T.M.; Methodology, M.L.; Investigation, M.L.; Data Curation, M.L.; Validation, M.L., I.L. and T.M.; Investigation, M.L.; Writing-Original Draft Preparation, M.L.; Writing—Review \& Editing, I.L. and T.M.; Visualization, M.L.; Supervision, I.L. and T.M.; Project Administration, T.M.; Resources, I.L. and T.M.; Funding Acquisition, M.L., I.L. and T.M. All authors have read and agreed to the published version of the manuscript.

Funding: This research received no external funding.

Acknowledgments: The APC was funded by Institute of Agriculture and Tourism and Faculty of Food Technology Osijek, Josip Juraj University of Osijek.

Conflicts of Interest: The authors declare no conflict of interest.

\section{References}

1. Oliveras-López, M.-J.; Berná, G.; Jurado-Ruiz, E.; La López-García de Serrana, H.; Martín, F. Consumption of extra-virgin olive oil rich in phenolic compounds has beneficial antioxidant effects in healthy human adults. J. Funct. Foods 2014, 10, 475-484. [CrossRef]

2. Rallo, L.; Díez, C.M.; Morales-Sillero, A.; Miho, H.; Priego-Capote, F.; Rallo, P. Quality of olives: A focus on agricultural preharvest factors. Sci. Hortic. 2018, 233, 491-509. [CrossRef]

3. Foscolou, A.; Critselis, E.; Panagiotakos, D. Olive oil consumption and human health: A narrative review. Maturitas 2018, 118, 60-66. [CrossRef] [PubMed]

4. International Olive Council: Madrid, Spain: World Olive Oil Figures. Olive Oil Production. Issue November 2020. Available online: https:/ / www.internationaloliveoil.org/wp-content/uploads/2020/12/HO-W901-23-11-2020-P.pdf (accessed on 10 September 2021).

5. International Olive Council, Madrid, Spain: EU Olive Oil Figures: Olive Oil Production. Issue November 2020. Available online: https:/ / www.internationaloliveoil.org/wp-content/uploads/2020/12/HO-CE901-23-11-2020-P-2.pdf (accessed on 10 September 2021).

6. Rossi, R. The EU Olive and Olive Oil Sector. Main Features, Challenges and Prospects; European Parliamentary Research Service: Brussels, Belgium, 2017. Available online: https:/ /www.europarl.europa.eu/RegData/etudes/BRIE/2017/608690/EPRS_BRI(20 17)608690_EN.pdf (accessed on 7 October 2021).

7. Servili, M.; Sordini, B.; Esposto, S.; Urbani, S.; Veneziani, G.; Di Maio, I.; Selvaggini, R.; Taticchi, A. biological activities of phenolic compounds of extra virgin olive oil. Antioxidants 2013, 3, 1-23. [CrossRef] [PubMed]

8. Piironen, V.; Lindsay, D.G.; Miettinen, T.A.; Toivo, J.; Lampi, A.-M. Plant sterols: Biosynthesis, biological function and their importance to human nutrition. J. Sci. Food Agric. 2000, 80, 939-966. [CrossRef]

9. Manai-Djebali, H.; Oueslati, I. Olive Oil Phytosterols and Human Health. In Olive Oil Sensory Characteristics, Composition and Importance in Human Health; Fritjof, T., Henning, B., Eds.; Nova Biomedical: New York, NY, USA, 2017; pp. 39-74, ISBN 978-1-53612-563-4.

10. European Economic Community. Commission Regulation (EEC) No 2568/91 of 11 July 1991 (and later modifications) on the characteristics of olive oil and olive-residue oil and the relevant methods of analysis. Off. J. Eur. Union 1991, L248, 1-83.

11. European Union. Regulation (EU) No 1151/2012 of the European parliament and of the Council of 21 November 2012 (and later modifications) on quality schemes for agricultural products and foodstuffs. Off. J. Eur. Union 2012, L343, 1-29.

12. Koutsaftakis, A.; Kotsifaki, F.; Stefanoudaki, E. Effect of extraction system, stage of ripeness, and kneading temperature on the sterol composition of virgin olive oils. J. Am. Oil Chem. Soc. 1999, 76, 1477-1481. [CrossRef]

13. Guillaume, C.; Ravetti, L.; Ray, D.L.; Johnson, J. Technological factors affecting sterols in Australian olive oils. J. Am. Oil Chem. Soc. 2012, 89, 29-39. [CrossRef]

14. Giuffrè, A.M.; Louadj, L.; Poiana, M.; Macario, A. Composition en stérols des huiles extraites d'olives de cultivars de la province de Reggio Calabria (Sud d'Italie). Riv. Ital. Sostanze Grasse 2012, 89, 177-183.

15. Schaller, H. New aspects of sterol biosynthesis in growth and development of higher plants. Plant Physiol. Biochem. 2004, 42, 465-476. [CrossRef] [PubMed]

16. Lukić, M.; Lukić, I.; Sladonja, B.; Piližota, V. Variability of 4-monomethylsterols and 4,4'-dimethylsterols in olive oil and their use as indicators of olive variety, ripening degree, and oil storage temperature. J. Agric. Food Chem. 2015, 63, 5499-5508. [CrossRef] [PubMed]

17. Feng, S.; Belwal, T.; Li, L.; Limwachiranon, J.; Liu, X.; Luo, Z. Phytosterols and their derivatives: Potential health-promoting uses against lipid metabolism and associated diseases, mechanism, and safety issues. Compr. Rev. Food Sci. Food Saf. 2020, 19, 1243-1267. [CrossRef] 
18. Mongrand, S.; Stanislas, T.; Bayer, E.M.F.; Lherminier, J.; Simon-Plas, F. Membrane rafts in plant cells. Trends Plant Sci. 2010, 15, 656-663. [CrossRef]

19. Oklestkova, J.; Rárová, L.; Kvasnica, M.; Strnad, M. Brassinosteroids: Synthesis and biological activities. Phytochem. Rev. 2015, 14, 1053-1072. [CrossRef]

20. Valitova, J.N.; Sulkarnayeva, A.G.; Minibayeva, F.V. Plant sterols: Diversity, biosynthesis, and physiological functions. Biochemistry 2016, 81, 819-834. [CrossRef]

21. Wojciechowski, Z.A. Biochemistry of phytosterol conjugates. In Physiology and Biochemistry of Sterols; Patterson, G.W., Nes, D.W., Eds.; American Oil Chemists' Society: Champaign, IL, USA, 1991; pp. 361-395, ISBN 9781003041023.

22. Goodwin, T.W. Biosynthesis of sterols. In The Biochemistry of Plants; Stumpf, P.K., Conn, E.E., Eds.; Academic Press: London, UK, 1980; pp. 485-507, ISBN 0-12-675404-7.

23. International Union of Pure and Applied Chemistry and International Union of Biochemistry Joint Commission on Biochemical Nomenclature. Nomenclature of steroids (recommendations 1989). Pure Appl. Chem. 1989, 61, 1783-1822. [CrossRef]

24. Belitz, H.-D.; Grosch, W.; Schieberle, P. Food Chemistry, 3rd ed.; Springer: Berlin/Heidelberg, Germany, 2004; pp. 224-225, ISBN 3-540-40818-5.

25. McCaskill, D.; Croteau, R. Some caveats for bioengineering terpenoid metabolism in plants. Trends Biotechnol. 1998, 16, 349-355. [CrossRef]

26. Hartmann, M. Plant sterols and the membrane environment. Trends Plant Sci. 1998, 3, 170-175. [CrossRef]

27. Rufino-Palomares, E.E.; Perez-Jimenez, A.; Reyes-Zurita, F.J.; Garcia-Salguero, L.; Mokhtari, K.; Herrera-Merchan, A.; Medina, P.; Peragon, J.; Lupianez, A.J. Anti-cancer and anti-angiogenic properties of various natural pentacyclic tri-terpenoids and some of their chemical derivatives. Curr. Org. Chem. 2015, 19, 919-947. [CrossRef]

28. Berger, A.; Jones, P.J.H.; Abumweis, S.S. Plant sterols: Factors affecting their efficacy and safety as functional food ingredients. Lipids Health Dis. 2004, 3, 5. [CrossRef]

29. Woyengo, T.A.; Ramprasath, V.R.; Jones, P.J.H. Anticancer effects of phytosterols. Eur. J. Clin. Nutr. 2009, 63, 813-820. [CrossRef] [PubMed]

30. Ramprasath, V.R.; Awad, A.B. Role of phytosterols in cancer prevention and treatment. J. AOAC Int. 2015, 98, 735-738. [CrossRef] [PubMed]

31. Peterson, D.W. Effect of soybean sterols in the diet on plasma and liver cholesterol in chicks. Proc. Soc. Exp. Biol. Med. 1951, 78, 143-147. [CrossRef] [PubMed]

32. Katan, M.B.; Grundy, S.M.; Jones, P.; Law, M.; Miettinen, T.; Paoletti, R. Efficacy and safety of plant stanols and sterols in the management of blood cholesterol levels. Mayo Clin. Proc. 2003, 78, 965-978. [CrossRef]

33. Ostlund, R.E. Phytosterols in human nutrition. Annu. Rev. Nutr. 2002, 22, 533-549. [CrossRef]

34. Expert Panel on Detection, Evaluation, and Treatment of High Blood Cholesterol in Adults (Adult Treatment Panel III). Executive summary of the third report of the National Cholesterol Education Program (NCEP). JAMA 2001, 285, 2486-2497. [CrossRef]

35. Cabral, C.E.; Klein, M.R.S.T. Phytosterols in the treatment of hypercholesterolemia and prevention of cardiovascular diseases. Arq. Bras. Cardiol. 2017, 109, 475-482. [CrossRef] [PubMed]

36. Rysz, J.; Franczyk, B.; Olszewski, R.; Banach, M.; Gluba-Brzozka, A. The use of plant sterols and stanols as lipid-lowering agents in cardiovascular disease. Curr. Pharm. Des. 2017, 23, 2488-2495. [CrossRef]

37. Othman, R.A.; Moghadasian, M.H. Beyond cholesterol-lowering effects of plant sterols: Clinical and experimental evidence of anti-inflammatory properties. Nutr. Rev. 2011, 69, 371-382. [CrossRef] [PubMed]

38. Nashed, B.; Yeganeh, B.; HayGlass, K.T.; Moghadasian, M.H. Antiatherogenic effects of dietary plant sterols are associated with inhibition of proinflammatory cytokine production in Apo E-KO mice. J. Nutr. 2005, 135, 2438-2444. [CrossRef]

39. Rocha, V.Z.; Ras, R.T.; Gagliardi, A.C.; Mangili, L.C.; Trautwein, E.A.; Santos, R.D. Effects of phytosterols on markers of inflammation: A systematic review and meta-analysis. Atherosclerosis 2016, 248, 76-83. [CrossRef]

40. Radika, M.K.; Viswanathan, P.; Anuradha, C.V. Nitric oxide mediates the insulin sensitizing effects of $\beta$-sitosterol in high fat diet-fed rats. Nitric Oxide 2013, 32, 43-53. [CrossRef] [PubMed]

41. Gupta, R.; Sharma, A.K.; Dobhal, M.P.; Sharma, M.C.; Gupta, R.S. Antidiabetic and antioxidant potential of $\beta$-sitosterol in streptozotocin-induced experimental hyperglycemia. J. Diabetes 2011, 3, 29-37. [CrossRef] [PubMed]

42. Saeidnia, S.; Manayi, A.; Gohari, A.R.; Abdollahi, M. The story of beta-sitosterol-A review. Eur. J. Med. Plants 2014, 4, 590-609. [CrossRef]

43. Bin Sayeed, M.S.; Karim, S.M.R.; Sharmin, T.; Morshed, M.M. Critical analysis on characterization, systemic effect, and therapeutic potential of beta-sitosterol: A plant-derived orphan phytosterol. Medicines 2016, 3, 29. [CrossRef] [PubMed]

44. Juan, M.E.; Wenzel, U.; Daniel, H.; Planas, J.M. Erythrodiol, a natural triterpenoid from olives, has antiproliferative and apoptotic activity in HT-29 human adenocarcinoma cells. Mol. Nutr. Food Res. 2008, 52, 595-599. [CrossRef] [PubMed]

45. Wang, L.; Wesemann, S.; Krenn, L.; Ladurner, A.; Heiss, E.H.; Dirsch, V.M.; Atanasov, A.G. Erythrodiol, an olive oil constituent, increases the half-life of ABCA1 and enhances cholesterol efflux from THP-1-derived macrophages. Front. Pharmacol. $2017,8,375$. [CrossRef]

46. Carmo, J.; Cavalcante-Araújo, P.; Silva, J.; Ferro, J.; Correia, A.C.; Lagente, V.; Barreto, E. Uvaol improves the functioning of fibroblasts and endothelial cells and accelerates the healing of cutaneous wounds in mice. Molecules 2020, 25, 4982. [CrossRef] [PubMed] 
47. Du, S.-Y.; Huang, H.-F.; Li, X.-Q.; Zhai, L.-X.; Zhu, Q.-C.; Zheng, K.; Song, X.; Xu, C.-S.; Li, C.-Y.; Li, Y.; et al. Anti-inflammatory properties of uvaol on DSS-induced colitis and LPS-stimulated macrophages. Chin. Med. 2020, 15, 43. [CrossRef]

48. Bonel-Pérez, G.C.; Pérez-Jiménez, A.; Gris-Cárdenas, I.; Parra-Pérez, A.M.; Lupiáñez, J.A.; Reyes-Zurita, F.J.; Siles, E.; Csuk, R.; Peragón, J.; Rufino-Palomares, E.E. Antiproliferative and pro-apoptotic effect of uvaol in human hepatocarcinoma HepG2 cells by affecting G0/G1 cell cycle arrest, ROS production and AKT/PI3K signaling pathway. Molecules 2020, 25, 4254. [CrossRef]

49. International Olive Council, Madrid, Spain: Trade Standard Applying to Olive Oils and Olive Pomace Oils; International Olive Council (COI/T.15/NC No 3/Rev. 16 June 2021). Available online: https:/ /www.internationaloliveoil.org/wp-content/uploads / 2021/07/COI-T15-NC3-REV-16-2021-_ENG.pdf (accessed on 7 October 2021).

50. Codex Alimentarius Commission, Geneva, Switzerland: Codex Standard for Named Vegetable Oils (Codex Stan 210-1999). Available online: http:/ / www.fao.org/fao-who-codexalimentarius/sh-proxy/en/?lnk=1\&url=https\%253A\%252F\%252Fworkspace. fao.org\%252Fsites\%252Fcodex\%252FStandards\%252FCXS\%2B210-1999\%252FCXS_210e.pdf (accessed on 7 October 2021).

51. Manai-Djebali, H.; Oueslati, I.; Martínez-Cañas, M.A.; Zarrouk, M.; Sánchez-Casas, J. Improvement of the sterol and triacylglycerol compositions of chemlali virgin olive oils through controlled crossing with mediterranean cultivars. J. Oleo Sci. 2018, 67, 379-388. [CrossRef] [PubMed]

52. Gutiérrez, F.; Jímenez, B.; Ruíz, A.; Albi, M.A. Effect of olive ripeness on the oxidative stability of virgin olive oil extracted from the varieties Picual and Hojiblanca and on the different components involved. J. Agric. Food Chem. 1999, 47, 121-127. [CrossRef] [PubMed]

53. Ranalli, A.; Angerosa, F. Integral centrifuges for olive oil extraction. The qualitative characteristics of products. J. Am. Oil Chem. Soc. 1996, 73, 417-422. [CrossRef]

54. Gutiérrez, F.; Varona, I.; Albi, M.A. Relation of acidity and sensory quality with sterol content of olive oil from stored fruit. J. Agric. Food Chem. 2000, 48, 1106-1110. [CrossRef]

55. Abu-Alruz, K.; Afaneh, I.A.; Quasem, J.M.; Hmidat, M.A.; Abbady, J.; Mazahreh, A.S. Factors affecting D-7-stigmastenol in Palestinian olive oil. J. Appl. Sci. 2011, 11, 797-805. [CrossRef]

56. Goudjil, H.; Torrado, S.; Fontecha, J.; Martínez-Castro, I.; Fraga, M.; Juárez, M. Composition of cholesterol and its precursors in ovine milk. Lait 2003, 83, 153-160. [CrossRef]

57. Mozzon, M.; Pacetti, D.; Frega, N.G.; Lucci, P. Crude palm oil from interspecific hybrid Elaeis oleifera $\times$ E. guineensis: Alcoholic constituents of unsaponifiable matter. J. Am. Oil Chem. Soc. 2015, 92, 717-724. [CrossRef]

58. Giuffrè, A.M.; Capocasale, M. Sterol composition of tomato (Solanum lycopersicum L.) seed oil: The effect of cultivar. Int. Food Res. J. 2016, 23, 116-122.

59. Grob, K.; Giuffré, A.M.; Leuzzi, U.; Mincione, B. Recognition of adulterated oil by direct analysis of the minor components. Eur. J. Lipid Sci. Technol. 1994, 8, 286-290. [CrossRef]

60. Alonso, L.; Fontecha, J.; Lozada, L.; Juárez, M. Determination of mixtures in vegetable oils and milk fat by analysis of sterol fraction by gas chromatography. J. Am. Oil Chem. Soc. 1997, 74, 131-135. [CrossRef]

61. Al-Ismail, K.M.; Alsaed, A.K.; Ahmad, R.; Al-Dabbas, M. Detection of olive oil adulteration with some plant oils by GLC analysis of sterols using polar column. Food Chem. 2010, 121, 1255-1259. [CrossRef]

62. Aparicio, R.; Morales, M.T.; Aparicio-Ruiz, R.; Tena, N.; García-González, D.L. Authenticity of olive oil: Mapping and comparing official methods and promising alternatives. Food Res. Int. 2013, 54, 2025-2038. [CrossRef]

63. Conte, L.; Bendini, A.; Valli, E.; Lucci, P.; Moret, S.; Maquet, A.; Lacoste, F.; Brereton, P.; García-González, D.L.; Moreda, W.; et al. Olive oil quality and authenticity: A review of current EU legislation, standards, relevant methods of analyses, their drawbacks and recommendations for the future. Trends Food Sci. Technol. 2020, 105, 483-493. [CrossRef]

64. Azadmard-Damirchi, S.; Dutta, P.C. Novel solid-phase extraction method to separate 4-desmethyl-, 4-monomethyl-, and 4,4'dimethylsterols in vegetable oils. J. Chromatogr. A 2006, 1108, 183-187. [CrossRef] [PubMed]

65. Mariani, C.; Bellan, G.; Morchio, G.; Pellegrino, A. Free and esterified minor components of olive and hazelnut oils. Their possible use in detecting blends. Note III. Riv. Ital. Sostanze Grasse 1999, 76, 297-305.

66. Cercaci, L.; Rodriguez-Estrada, M.; Lercker, G. Solid-phase extraction-thin-layer chromatography-gas chromatography method for the detection of hazelnut oil in olive oils by determination of esterified sterols. J. Chromatogr. A 2003, 985, 211-220. [CrossRef]

67. International Olive Council. Determination of the Sterol Composition and Content and Alcoholic Compounds by Capillary Gas Chromatography (COI/T.20/ Doc. No 26/Rev., 5 June 2020); International Olive Council: Madrid, Spain, 2020.

68. Codex Alimentarius Commission, Geneva, Switzerland. Codex Standard for Olive Oils and Olive Pomace Oils (Codex Stan 33. 1981). Available online: http:/ / www.fao.org/fao-who-codexalimentarius/sh-proxy / en / ?lnk=1\&url=https $\% 253 \mathrm{~A} \% 252 \mathrm{~F} \% 25$ 2Fworkspace.fao.org\%252Fsites\%252Fcodex\%252FStandards\%252FCXS\%2B33-1981\%252FCXS_033e.pdf (accessed on 7 October 2021).

69. International Organization for Standardization. Determination of Individual and Total Sterols Contents, Gas Chromatographic Method, Part 2: Olive Oils and Olive Pomace Oils (ISO 12228-2:2014); International Organisation for Standardization: Geneva, Switzerland, 2014.

70. American Oil Chemists' Society. Determination of the composition of the sterol fraction of animal and vegetable oils and fats by TLC and capillary GLC (AOCS Official Method Ch 6-91). In Methods and Recommended Practices of the AOCS, 6th ed.; Firestone, D., Ed.; AOCS Press: Champaign, IL, USA, 1997; pp. 1-5. 
71. Bianchi, G.; Giansante, L.; Shaw, A.; Kell, D.B. Chemometric criteria for the characterisation of Italian protected denomination of origin (DOP) olive oils from their metabolic profiles. Eur. J. Lipid Sci. Technol. 2001, 103, 141-150. [CrossRef]

72. Giacometti, J. Determination of aliphatic alcohols, squalene, alpha-tocopherol and sterols in olive oils: Direct method involving gas chromatography of the unsaponifiable fraction following silylation. Analyst 2001, 126, 472-475. [CrossRef]

73. Giacometti, J.; Milin, Č.; Giacometti, F.; Ciganj, Z. Characterisation of monovarietal oolive oils obtained from Croatian cvs. Drobnica and Buza during the ripening period. Foods 2018, 7, 188. [CrossRef] [PubMed]

74. Navas-López, J.F.; Cano, J.; La Rosa, R.; de Velasco, L.; León, L. Genotype by environment interaction for oil quality components in olive tree. Eur. J. Agron. 2020, 119, 126115. [CrossRef]

75. Olmo-García, L.; Polari, J.J.; Li, X.; Bajoub, A.; Fernández-Gutiérrez, A.; Wang, S.C.; Carrasco-Pancorbo, A. Deep insight into the minor fraction of virgin olive oil by using LC-MS and GC-MS multi-class methodologies. Food Chem. 2018, 261, 184-193. [CrossRef] [PubMed]

76. Almeida, C.A.S.; Baggio, S.R.; Mariutti, L.R.B.; Bragagnolo, N. One-step rapid extraction of phytosterols from vegetable oils. Food Res. Int. 2020, 130, 108891. [CrossRef] [PubMed]

77. Purcaro, G.; Barp, L.; Beccaria, M.; Conte, L.S. Fingerprinting of vegetable oil minor components by multidimensional comprehensive gas chromatography with dual detection. Anal. Bioanal. Chem. 2015, 407, 309-319. [CrossRef] [PubMed]

78. Aloisi, I.; Zoccali, M.; Dugo, P.; Tranchida, P.Q.; Mondello, L. Fingerprinting of the unsaponifiable fraction of vegetable oils by using cryogenically-modulated comprehensive two-dimensional gas chromatography-high resolution time-of-flight mass spectrometry. Food Anal. Methods 2020, 13, 1523-1529. [CrossRef]

79. Olmo-García, L.; Carrasco-Pancorbo, A. Chromatography-MS based metabolomics applied to the study of virgin olive oil bioactive compounds: Characterization studies, agro-technological investigations and assessment of healthy properties. Trends Anal. Chem. 2021, 135, 116153. [CrossRef]

80. Cert, A.; Moreda, W.; García-Moreno, J. Determination of sterols and triterpenic dialcohols in olive oils using HPLC separation and GC analysis. Standardization of the analytical method. Grasas Aceites 1997, 48, 207-218. [CrossRef]

81. Cañabate-Díaz, B.; Segura Carretero, A.; Fernández-Gutiérrez, A.; Belmonte Vega, A.; Garrido Frenich, A.; Martínez Vidal, J.L.; Duran Martos, J. Separation and determination of sterols in olive oil by HPLC-MS. Food Chem. 2007, 102, 593-598. [CrossRef]

82. Lerma-García, M.J.; Concha-Herrera, V.; Herrero-Martínez, J.M.; Simó-Alfonso, E.F. Classification of extra virgin olive oils produced at La Comunitat Valenciana according to their genetic variety using sterol profiles established by high-performance liquid chromatography with mass spectrometry detection. J. Agric. Food Chem. 2009, 57, 10512-10517. [CrossRef] [PubMed]

83. Mo, S.; Dong, L.; Hurst, W.J.; van Breemen, R.B. Quantitative analysis of phytosterols in edible oils using APCI liquid chromatography-tandem mass spectrometry. Lipids 2013, 48, 949-956. [CrossRef]

84. Abdallah, M.; Vergara-Barberán, M.; Lerma-García, M.J.; Herrero-Martínez, J.M.; Zarrouk, M.; Guerfel, M.; Simó-Alfonso, E.F. Sterol profiles of Tunisian virgin olive oils: Classification among different cultivars and maturity indexes. Eur. Food Res. Technol. 2018, 244, 675-684. [CrossRef]

85. Lerma-García, M.J.; Simó-Alfonso, E.F.; Méndez, A.; Lliberia, J.L.; Herrero-Martínez, J.M. Classification of extra virgin olive oils according to their genetic variety using linear discriminant analysis of sterol profiles established by ultra-performance liquid chromatography with mass spectrometry detection. Food Res. Int. 2011, 44, 103-108. [CrossRef]

86. Ghisoni, S.; Lucini, L.; Angilletta, F.; Rocchetti, G.; Farinelli, D.; Tombesi, S.; Trevisan, M. Discrimination of extra-virgin-olive oils from different cultivars and geographical origins by untargeted metabolomics. Food Res. Int. 2019, 121, 746-753. [CrossRef] [PubMed]

87. Mohamed, M.B.; Rocchetti, G.; Montesano, D.; Ali, S.B.; Guasmi, F.; Grati-Kamoun, N.; Lucini, L. Discrimination of Tunisian and Italian extra-virgin olive oils according to their phenolic and sterolic fingerprints. Food Res. Int. 2018, 106, 920-927. [CrossRef] [PubMed]

88. Nestola, M.; Schmidt, T.C. Fully automated determination of the sterol composition and total content in edible oils and fats by online liquid chromatography-gas chromatography-flame ionization detection. J. Chromatogr. A 2016, 1463, 136-143. [CrossRef] [PubMed]

89. Hatzakis, E.; Dagounakis, G.; Agiomyrgianaki, A.; Dais, P. A facile NMR method for the quantification of total, free and esterified sterols in virgin olive oil. Food Chem. 2010, 122, 346-352. [CrossRef]

90. Özdemir, İ.S.; Dağ, Ç.; Özinanç, G.; Suçsoran, Ö.; Ertaş, E.; Bekiroğlu, S. Quantification of sterols and fatty acids of extra virgin olive oils by FT-NIR spectroscopy and multivariate statistical analyses. LWT 2018, 91, 125-132. [CrossRef]

91. Panagiotopoulou, P.M.; Tsimidou, M. Solid phase extraction: Applications to the chromatographic analysis of vegetable oils and fats. Grasas Aceites 2002, 53, 84-95. [CrossRef]

92. Verleyen, T.; Forcades, M.; Verhe, R.; Dewettinck, K.; Huyghebaert, A.; De Greyt, W. Analysis of free and esterified sterols in vegetable oils. J. Am. Oil Chem. Soc. 2002, 79, 117-122. [CrossRef]

93. Grob, K.; Lanfranchi, M.; Mariani, F. Determination of free and esterified sterols and of wax esters in oils and fats by coupled liquid chromatography-gas chromatography. J. Chromatogr. A 1989, 471, 397-405. [CrossRef]

94. Mariani, C.; Bellan, G.; Lestini, E.; Aparicio, R. The detection of the presence of hazelnut oil in olive oil by free and esterified sterols. Eur. Food Res. Technol. 2006, 223, 655-661. [CrossRef]

95. Cunha, S.S.; Fernandes, J.O.; Oliveira, M.B.P.P. Quantification of free and esterified sterols in Portuguese olive oils by solid-phase extraction and gas chromatography-mass spectrometry. J. Chromatogr. A 2006, 1128, 220-227. [CrossRef] [PubMed] 
96. Valli, E.; Milani, A.; Srbinovska, A.; Moret, E.; Moret, S.; Bendini, A.; Moreda, W.; Toschi, T.G.; Lucci, P. In-house validation of an SPE-GC-FID method for the detection of free and esterified hydroxylated minor compounds in virgin olive oils. Foods 2021, 10, 1260. [CrossRef] [PubMed]

97. Gómez-Coca, R.B.; del Pérez-Camino, C.M.; Moreda, W. On the glucoside analysis: Simultaneous determination of free and esterified steryl glucosides in olive oil. Detailed analysis of standards as compulsory first step. Food Chem. 2013, 141, 1273-1280. [CrossRef]

98. Rivera del Álamo, R.M.; Fregapane, G.; Aranda, F.; Gómez-Alonso, S.; Salvador, M.D. Sterol and alcohol composition of Cornicabra virgin olive oil: The campesterol content exceeds the upper limit of $4 \%$ established by EU regulations. Food Chem. 2004, 84, 533-537. [CrossRef]

99. Sánchez Casas, J.; Osorio Bueno, E.; Montaño García, A.M.; Martinez Cano, M. Sterol and erythrodiol + uvaol content of virgin olive oils from cultivars of Extremadura (Spain). Food Chem. 2004, 87, 225-230. [CrossRef]

100. Galeano Diaz, T.; Durán Merás, I.; Sánchez Casas, J.; Alexandre Franco, M.F. Characterization of virgin olive oils according to its triglycerides and sterols composition by chemometric methods. Food Control 2005, 16, 339-347. [CrossRef]

101. López-Cortés, I.; Salazar-García, D.C.; Velázquez-Martí, B.; Salazar, D.M. Chemical characterization of traditional varietal olive oils in East of Spain. Food Res. Int. 2013, 54, 1934-1940. [CrossRef]

102. Pardo, J.E.; Sena, E.; Cuesta, M.A.; Granell, J.D.; Valiente, J.; Alvarez-Ortí, M. Evaluation of potential and real quality of virgin olive oil from "Campos de Hellín" (Albacete, Spain). J. Am. Oil Chem. Soc. 2013, 90, 851-862. [CrossRef]

103. Gómez-Coca, R.B.; Fernandes, G.D.; Del Aguila-Sánchez, C.; Del Pérez-Camino, M.C.; Moreda, W. Comparative study of phytosterol derivatives in monovarietal olive oils. J. Agric. Food Chem. 2014, 62, 5669-5674. [CrossRef]

104. Fuentes, M.; de Miguel, C.; Ranalli, A.; Franco, M.N.; Martínez, M.; Martín-Vertedor, D. Chemical composition and sensory evaluation of virgin olive oils from "Morisca" and "Carrasqueña" olive varieties. Grasas Aceites 2015, 66, e061. [CrossRef]

105. Sena-Moreno, E.; Alvarez-Ortí, M.; Zied, D.C.; Pardo-Giménez, A.; Pardo, J.E. Olive oils from Campos de Hellin (Spain) exhibit significant varietal differences in fatty acid composition, sterol fraction, and oxidative stability. Eur. J. Lipid Sci. Technol. 2015, 117, 967-975. [CrossRef]

106. Salazar-García, D.C.; Malheiro, R.; Pereira, J.A.; Lopéz-Cortés, I. Unexplored olive cultivars from the Valencian Community (Spain): Some chemical characteristics as a valorization strategy. Eur. Food Res. Technol. 2019, 245, 325-334. [CrossRef]

107. Pardo, J.E.; Tello, J.; Suárez, M.; Rabadán, A.; de Miguel, C.; Álvarez-Orti, M. Variety characterization and influence of olive maturity in virgin olive oils from the area assigned to the Protected Designation of Origin "Aceite de la Alcarria" (Spain). Agronomy 2020, 10, 38. [CrossRef]

108. Kyçyk, O.; Aguilera, M.P.; Gaforio, J.J.; Jiménez, A.; Beltrán, G. Sterol composition of virgin olive oil of forty-three olive cultivars from the World Collection Olive Germplasm Bank of Cordoba. J. Sci. Food Agric. 2016, 96, 4143-4150. [CrossRef] [PubMed]

109. Allouche, Y.; Jiménez, A.; Uceda, M.; Aguilera, M.P.; Gaforio, J.J.; Beltrán, G. Triterpenic content and chemometric analysis of virgin olive oils from forty olive cultivars. J. Agric. Food Chem. 2009, 57, 3604-3610. [CrossRef] [PubMed]

110. Stefanoudaki, E.; Kotsifaki, F.; Koutsaftakis, A. Sensory and chemical profiles of three European olive varieties (Olea europea L); an approach for the characterisation and authentication of the extracted oils. J. Sci. Food Agric. 2000, 80, 381-389. [CrossRef]

111. Ranalli, A.; Pollastri, L.; Contento, S.; Di Loreto, G.; Iannucci, E.; Lucera, L.; Russi, F. Sterol and alcohol components of seed, pulp and whole olive fruit oils. Their use to characterise olive fruit variety by multivariates. J. Sci. Food Agric. 2002, 82, 854-859. [CrossRef]

112. Giansante, L.; Di Vincenzo, D.; Bianchi, G. Classification of monovarietal Italian olive oils by unsupervised (PCA) and supervised (LDA) chemometrics. J. Sci. Food Agric. 2003, 83, 905-911. [CrossRef]

113. Marini, F.; Balestrieri, F.; Bucci, R.; Magrì, A.D.; Magrì, A.L.; Marini, D. Supervised pattern recognition to authenticate Italian extra virgin olive oil varieties. Chemometr. Intell. Lab. Syst. 2004, 73, 85-93. [CrossRef]

114. Sivakumar, G.; Bati, C.; Perri, E.; Uccella, N. Gas chromatography screening of bioactive phytosterols from mono-cultivar olive oils. Food Chem. 2006, 95, 525-528. [CrossRef]

115. Giuffrè, A.M.; Louadj, L. Influence of crop season and cultivar on sterol composition of monovarietal olive oils in Reggio Calabria (Italy). Czech J. Food Sci. 2013, 31, 256-263. [CrossRef]

116. Deiana, P.; Santona, M.; Dettori, S.; Molinu, M.G.; Dore, A.; Culeddu, N.; Azara, E.; Naziri, E.; Tsimidou, M.Z. Can all the Sardinian varieties support the PDO “Sardegna” virgin olive oil? Eur. J. Lipid Sci. Technol. 2019, 121, 1800135. [CrossRef]

117. Vekiari, S.A.; Oreopoulou, V.; Kourkoutas, Y.; Kamoun, N.; Msallem, M.; Psimouli, V.; Arapoglou, D. Characterization and seasonal variation of the quality of virgin olive oil of the Throumbolia and Koroneiki varieties from southern Greece. Grasas Aceites 2010, 61, 221-231. [CrossRef]

118. Skiada, V.; Tsarouhas, P.; Varzakas, T. Preliminary study and observation of "Kalamata PDO" extra virgin olive oil, in the Messinia region, southwest of Peloponnese (Greece). Foods 2019, 8, 610. [CrossRef] [PubMed]

119. Skiada, V.; Tsarouhas, P.; Varzakas, T. Comparison and discrimination of two major monocultivar extra virgin olive oils in the southern region of Peloponnese, according to specific compositional/traceability markers. Foods 2020, 9, 155. [CrossRef]

120. Skiada, V.; Agriopoulou, S.; Tsarouhas, P.; Katsaris, P.; Stamatelopoulou, E.; Varzakas, T. Evaluation and origin discrimination of two monocultivar extra virgin olive oils, cultivated in the coastline part of North-Western Greece. Appl. Sci. 2020, 10, 6733. [CrossRef] 
121. Ilyasoglu, H.; Ozcelik, B.; van Hoed, V.; Verhe, R. Characterization of Aegean olive oils by their minor compounds. J. Am. Oil Chem. Soc. 2010, 87, 627-636. [CrossRef]

122. Yorulmaz, A.; Yavuz, H.; Tekin, A. Characterization of Turkish olive oils by triacylglycerol structures and sterol profiles. J. Am. Oil Chem. Soc. 2014, 91, 2077-2090. [CrossRef]

123. Bozdogan Konuskan, D.; Mungan, B. Effects of variety, maturation and growing region on chemical properties, fatty acid and sterol compositions of virgin olive oils. J. Am. Oil Chem. Soc. 2016, 93, 1499-1508. [CrossRef]

124. Yorulmaz, H.O.; Konuskan, D.B. Antioxidant activity, sterol and fatty acid compositions of Turkish olive oils as an indicator of variety and ripening degree. J. Food Sci. Technol. 2017, 54, 4067-4077. [CrossRef] [PubMed]

125. Demirag, O.; Konuskan, D.B. Quality properties, fatty acid and sterol compositions of East Mediterranean region olive oils. J. Oleo Sci. 2021, 70, 51-58. [CrossRef] [PubMed]

126. Haddada, F.M.; Manaï, H.; Oueslati, I.; Daoud, D.; Sánchez, J.; Osorio, E.; Zarrouk, M. Fatty acid, triacylglycerol, and phytosterol composition in six Tunisian olive varieties. J. Agric. Food Chem. 2007, 55, 10941-10946. [CrossRef] [PubMed]

127. Oueslati, I.; Manai, H.; Haddada, F.M.; Daoud, D.; Sánchez, J.; Osorio, E.; Zarrouk, M. Sterol, Triterpenic dialcohol, and triacylglycerol compounds of extra virgin olive oils from some Tunisian varieties grown in the region of Tataouine. Food Sci. Technol. Int. 2009, 15, 5-13. [CrossRef]

128. Krichène, D.; Allalout, A.; Salvador, M.D.; Fregapane, G.; Zarrouk, M. Fatty acids, volatiles, sterols and triterpenic alcohols of six monovarietal Tunisian virgin olive oils. Eur. J. Lipid Sci. Technol. 2010, 112, 400-409. [CrossRef]

129. Manai-Djebali, H.; Krichène, D.; Ouni, Y.; Gallardo, L.; Sánchez, J.; Osorio, E.; Daoud, D.; Guido, F.; Zarrouk, M. Chemical profiles of five minor olive oil varieties grown in central Tunisia. J. Food Compost. Anal. 2012, 27, 109-119. [CrossRef]

130. Alves, M.R.; Cunha, S.C.; Amaral, J.S.; Pereira, J.A.; Oliveira, M.B. Classification of PDO olive oils on the basis of their sterol composition by multivariate analysis. Anal. Chim. Acta 2005, 549, 166-178. [CrossRef]

131. Matos, L.C.; Cunha, S.C.; Amaral, J.S.; Pereira, J.A.; Andrade, P.B.; Seabra, R.M.; Oliveira, B.P. Chemometric characterization of three varietal olive oils (Cvs. Cobrançosa, Madural and Verdeal Transmontana) extracted from olives with different maturation indices. Food Chem. 2007, 102, 406-414. [CrossRef]

132. Boulkroune, H.; Lazzez, A.; Guissous, M.; Bellik, Y.; Smaoui, S.; Kamoun, N.G.; Madani, T. Characterization of sterolic and alcoholic fractions of some Algerian olive oils according to the variety and ripening stage. OCL J. 2017, 24, A502. [CrossRef]

133. Ceci, L.N.; Carelli, A.A. Characterization of monovarietal argentinian olive oils from new productive zones. J. Am. Oil Chem. Soc. 2007, 84, 1125-1136. [CrossRef]

134. Mailer, R.J.; Ayton, J.; Graham, K. The influence of growing region, cultivar and harvest timing on the diversity of Australian olive oil. J. Am. Oil Chem. Soc. 2010, 87, 877-884. [CrossRef]

135. Olmo-García, L.; Polari, J.J.; Li, X.; Bajoub, A.; Fernández-Gutiérrez, A.; Wang, S.C.; Carrasco-Pancorbo, A. Study of the minor fraction of virgin olive oil by a multi-class GC-MS approach: Comprehensive quantitative characterization and varietal discrimination potential. Food Res. Int. 2019, 125, 108649. [CrossRef]

136. Noorali, M.; Barzegar, M.; Sahari, M.A. Sterol and fatty acid compositions of olive oil as an indicator of cultivar and growing area. J. Am. Oil Chem. Soc. 2014, 91, 1571-1581. [CrossRef]

137. Lukić, M.; Lukić, I.; Krapac, M.; Sladonja, B.; Piližota, V. Sterols and triterpene diols in olive oil as indicators of variety and degree of ripening. Food Chem. 2013, 136, 251-258. [CrossRef] [PubMed]

138. Brkic Bubola, K.; Valenčič, V.; Bucar-Miklavčič, M.; Krapac, M.; Lukic, M.; Setic, E.; Sladonja, B. Sterol, triterpen dialcohol and fatty acid profile of less-and well-known Istrian monovarietal olive oil. Croat. J. Food Sci. Technol. 2018, 10, 118-122. [CrossRef]

139. Ajana, H.; El Antari, A. Fatty acids and sterols evolution during the ripening of olives from the Moroccan Picholine cultivar. Grasas Aceites 1998, 49, 405-410. [CrossRef]

140. Aparicio, R.; Luna, G. Characterisation of monovarietal virgin olive oil. Eur. J. Lipid Sci. Technol. 2002, 104, 614-627. [CrossRef]

141. Salvador, M.D.; Aranda, F.; Fregapane, G. Chemical composition of commercial cornicabra virgin olive oil from $1995 / 96$ and 1996/97 crops. J. Am. Oil Chem. Soc. 1998, 75, 1305-1311. [CrossRef]

142. Srigley, C.T.; Oles, C.J.; Kia, A.R.F.; Mossoba, M.M. Authenticity assessment of extra virgin olive oil: Evaluation of desmethylsterols and triterpene dialcohols. J. Am. Oil Chem. Soc. 2016, 93, 171-181. [CrossRef]

143. Beltrán, G.; Del Río, C.; Sánchez, S.; Martínez, L. Seasonal changes in olive fruit characteristics and oil accumulation during ripening process. J. Sci. Food Agric. 2004, 84, 1783-1790. [CrossRef]

144. Avidan, B.; Meni, Y.; Tsur, N. Composition and morphology changes in olive fruit as indication of maturation. Adv. Hortic. Sci. 2007, 21, 3-8.

145. Sönmez, A.; Özdikicierler, O.; Saygin Gümüşkesen, A. Evaluation of olive oil quality during the ripening of the organic cultivated olives and multivariate discrimination of the variety with a chemometric approach. Riv. Ital. Sostanze Grasse 2018, 95, $173-181$.

146. Fuentes de Mendoza, M.; de Miguel Gordillo, C.; Marín Expóxito, J.; Sánchez Casas, J.; Martínez Cano, M.; Martín Vertedor, D.; Franco Baltasar, M.N. Chemical composition of virgin olive oils according to the ripening in olives. Food Chem. 2013, 141, 2575-2581. [CrossRef] [PubMed]

147. Lazzez, A.; Perri, E.; Caravita, M.A.; Khlif, M.; Cossentini, M. Influence of olive maturity stage and geographical origin on some minor components in virgin olive oil of the Chemlali variety. J. Agric. Food Chem. 2008, 56, 982-988. [CrossRef] [PubMed]

148. Mena, C.; González, A.Z.; Olivero-David, R.; Pérez-Jiménez, M.Á. Characterization of 'Castellana' virgin olive oils with regard to olive ripening. Horttechnology 2018, 28, 48-57. [CrossRef] 
149. Taticchi, A.; Selvaggini, R.; Esposto, S.; Sordini, B.; Veneziani, G.; Servili, M. Physicochemical characterization of virgin olive oil obtained using an ultrasound-assisted extraction at an industrial scale: Influence of olive maturity index and malaxation time. Food Chem. 2019, 289, 7-15. [CrossRef]

150. Yorulmaz, A.; Erinc, H.; Tekin, A. Changes in olive and olive oil characteristics during maturation. J. Am. Oil Chem. Soc. 2013, 90, 647-658. [CrossRef]

151. Chtourou, F.; Jabeur, H.; Lazzez, A.; Bouaziz, M. Characterization and discrimination of Oueslati virgin olive oils from adult and young trees in different ripening stages using sterols, pigments, and alcohols in tandem with chemometrics. J. Agric. Food Chem. 2017, 65, 3512-3522. [CrossRef] [PubMed]

152. Lazzez, A.; Vichi, S.; Kammoun, N.G.; Arous, M.N.; Khlif, M.; Romero, A.; Cossentini, M. A four year study to determine the optimal harvesting period for Tunisian Chemlali olives. Eur. J. Lipid Sci. Technol. 2011, 113, 796-807. [CrossRef]

153. Kaliora, A.C.; Artemiou, A.; Giogios, I.; Kalogeropoulos, N. The impact of fruit maturation on bioactive microconstituents, inhibition of serum oxidation and inflammatory markers in stimulated PBMCs and sensory characteristics of Koroneiki virgin olive oils from Messenia, Greece. Food Funct. 2013, 4, 1185-1194. [CrossRef] [PubMed]

154. Wang, Y.; Yu, L.; Zhao, A.; Karrar, E.; Zhang, H.; Jin, Q.; Wu, G.; Yang, X.; Chen, L.; Wang, X. Quality characteristics and antioxidant activity during fruit ripening of three monovarietal olive oils cultivated in china. J. Am. Oil Chem. Soc. 2021, 98, 229-240. [CrossRef]

155. Salvador, M.D.; Aranda, F.; Fregapane, G. Influence of fruit ripening on 'Cornicabra' virgin olive oil quality A study of four successive crop seasons. Food Chem. 2001, 73, 45-53. [CrossRef]

156. Fernández-Cuesta, A.; León, L.; Velasco, L.; De La Rosa, R. Changes in squalene and sterols associated with olive maturation. Food Res. Int. 2013, 54, 1885-1889. [CrossRef]

157. Uceda, M.; Frias, L. Harvest dates: Evolution of the fruit oil content, oil composition and oil quality. In Proceedings of the 2nd Seminario Oleícola Internacional, Cordoba, Spain, 6 October 1975; pp. 125-128.

158. Giuffrè, A.M. Biometric evaluation of twelve olive cultivars under rainfed conditions in the region of Calabria, South Italy. Emir. J. Food Agric. 2017, 29, 696-709. [CrossRef]

159. Sakouhi, F.; Absalon, C.; Harrabi, S.; Vitry, C.; Sebei, K.; Boukhchina, S.; Fouquet, E.; Kallel, H. Dynamic accumulation of 4-desmethylsterols and phytostanols during ripening of Tunisian Meski olives (Olea europea L.). Food Chem. 2009, 112, 897-902. [CrossRef]

160. Ranalli, A.; Tombesi, A.; Ferrante, M.L.; De Mattia, G. Respiratory rate of olive drupes during their ripening cycle and quality of oil extracted. J. Sci. Food Agric. 1998, 77, 359-367. [CrossRef]

161. Sakouhi, F.; Herchi, W.; Sbei, K.; Absalon, C.; Boukhchina, S. Characterisation and accumulation of squalene and n-alkanes in developing Tunisian Olea europaea L. fruits. Int. J. Food Sci. Technol. 2011, 46, 2281-2286. [CrossRef]

162. Orozco-Solano, M.; Ruiz-Jimenez, J.; Luque De Castro, M.D. Characterization of fatty alcohol and sterol fractions in olive tree. J. Agric. Food Chem. 2010, 58, 7539-7546. [CrossRef] [PubMed]

163. Ferreiro, L.; Aparicio, R. Influencia de la altitud en la composición química de los aceites de oliva vírgenes de Andalucía. Ecuaciones matemáticas de clasificación. Grasas Aceites 1992, 43, 149-156. [CrossRef]

164. Ranalli, A.; de Mattia, G.; Patumi, M.; Proietti, P. Quality of virgin olive oil as influenced by origin area. Grasas Aceites 1999, 50, 249-259. [CrossRef]

165. Anastasopoulos, E.; Kalogeropoulos, N.; Kaliora, A.C.; Kountouri, A.; Andrikopoulos, N.K. The influence of ripening and crop year on quality indices, polyphenols, terpenic acids, squalene, fatty acid profile, and sterols in virgin olive oil (Koroneiki cv.) produced by organic versus non-organic cultivation method. Int. J. Food Sci. Technol. 2011, 46, 170-178. [CrossRef]

166. Ben Temime, S.; Manai, H.; Methenni, K.; Baccouri, B.; Abaza, L.; Daoud, D.; Casas, J.S.; Bueno, E.O.; Zarrouk, M. Sterolic composition of Chétoui virgin olive oil: Influence of geographical origin. Food Chem. 2008, 110, 368-374. [CrossRef] [PubMed]

167. Alberghina, G.; Caruso, L.; Fisichella, S.; Musumarra, G. Geographical classification of sicilian olive oils in terms of sterols and fatty acids content. J. Sci. Food Agric. 1991, 56, 445-455. [CrossRef]

168. Longobardi, F.; Ventrella, A.; Casiello, G.; Sacco, D.; Tasioula-Margari, M.; Kiritsakis, A.K.; Kontominas, M.G. Characterisation of the geographical origin of Western Greek virgin olive oils based on instrumental and multivariate statistical analysis. Food Chem. 2012, 133, 169-175. [CrossRef]

169. Longobardi, F.; Ventrella, A.; Casiello, G.; Sacco, D.; Catucci, L.; Agostiano, A.; Kontominas, M.G. Instrumental and multivariate statistical analyses for the characterisation of the geographical origin of Apulian virgin olive oils. Food Chem. 2012, 133, 579-584. [CrossRef]

170. Bajoub, A.; Hurtado-Fernández, E.; Ajal, E.A.; Fernández-Gutiérrez, A.; Carrasco-Pancorbo, A.; Ouazzani, N. Quality and chemical profiles of monovarietal north Moroccan olive oils from "Picholine Marocaine" cultivar: Registration database development and geographical discrimination. Food Chem. 2015, 179, 127-136. [CrossRef]

171. Giacalone, R.; Giuliano, S.; Gulotta, E.; Monfreda, M.; Presti, G. Origin assessment of EV olive oils by esterified sterols analysis. Food Chem. 2015, 188, 279-285. [CrossRef]

172. Inglese, P.; Barone, E.; Gullo, G. The effect of complementary irrigation on fruit growth, ripening pattern and oil characteristics of olive (Olea europaea L.) cv. Carolea. J. Hortic. Sci. 1996, 71, 257-263. [CrossRef]

173. Stefanoudaki, E.; Chartzoulakis, K.; Koutsaftakis, A.; Kotsifaki, F. Effect of drought stress on qualitative characteristics of olive oil of cv Koroneiki. Grasas Aceites 2001, 52, 202-206. [CrossRef] 
174. Stefanoudaki, E.; Williams, M.; Chartzoulakis, K.; Harwood, J. Effect of irrigation on quality attributes of olive oil. J. Agric. Food Chem. 2009, 57, 7048-7055. [CrossRef]

175. Barone, E.; Gullo, G.; Zappia, R.; Inglese, P. Effect of crop load on fruit ripening and olive oil (Olea europea L.) quality. J. Hortic. Sci. 1994, 69, 67-73. [CrossRef]

176. Di Giovacchino, L.; Sestili, S.; Di Vincenzo, D. Influence of olive processing on virgin olive oil quality. Eur. J. Lipid Sci. Technol. 2002, 104, 587-601. [CrossRef]

177. Ranalli, A.; Ferrante, M.L.; de Mattia, G.; Costantini, N. Analytical evaluation of virgin olive oil of first and second extraction. J. Agric. Food Chem. 1999, 47, 417-424. [CrossRef]

178. Cercaci, L.; Passalacqua, G.; Poerio, A.; Rodriguez-Estrada, M.T.; Lercker, G. Composition of total sterols (4-desmethyl-sterols) in extravirgin olive oils obtained with different extraction technologies and their influence on the oil oxidative stability. Food Chem. 2007, 102, 66-76. [CrossRef]

179. Thanh, T.T.; Vergnes, M.-F.; Kaloustian, J.; El-Moselhy, T.F.; Amiot-Carlin, M.-J.; Portugal, H. Effect of storage and heating on phytosterol concentrations in vegetable oils determined by GC/MS. J. Sci. Food Agric. 2006, 86, 220-225. [CrossRef] 